\title{
Supporting Information for: \\ What is the optimal size of quantum region in embedding calculations of two-photon absorption spectra of fluorescent proteins?
}

Dawid Grabarek, Tadeusz Andruniów*

$$
\text { E-mail:tadeusz.andruniow@pwr.edu.pl }
$$

\section{Advanced Materials Engineering and Modelling Group \\ Wroclaw University of Science and Technology, \\ Wyb. Wyspiańskiego 27, 50-370 Wroclaw, Poland}

\section{Contents}

S1 Details of MD simulations protocol $\quad$ S2

$\mathrm{S} 1.1$ Classical MD simulations . . . . . . . . . . . . . . . . . . . . . . S2

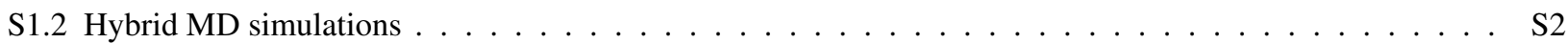

S2 FP models structure $\quad$ S3

S3 Composition of QM subsystems $\quad$ S6

S4 Full set of OPA and TPA spectra
S15

S5 $\triangle E, \sigma^{T P A}$ and $f$ values obtained for different models of FPs $\quad$ S24

S6 Remaining supporting information $\quad \mathbf{S 4 0}$

S6.1 Comparison of $\triangle E, \sigma^{T P A}$ and $f$ values obtained with BHandHLYP and CAM-B3LYP functionals for chosen models of avGFP-n and Citrine . . . . . . . . . . . . . . . . . . . . . . . S40

S6.2 Comparison of $\triangle E, \sigma^{T P A}$ and $f$ values obtained in PE approximation with water molecules included in $3.0,5.0$, and $7.0 \AA$ A radius from protein . . . . . . . . . . . . . . . . . . . . . S44

S6.3 Role of effective electrostatic field in OPA and TPA spectra tuning . . . . . . . . . . . . . . . . S45

*To whom correspondence should be addressed 


\section{S1 Details of MD simulations protocol}

\section{S1.1 Classical MD simulations}

Starting from the solvated protein system as described in the main text, the classical MD simulation protocol is following:

- 1000 steps of steepest-descent minimization with all protein atoms restrained followed by another 1000 minimization steps with all heavy protein atoms restrained. The force constant of $2000 \mathrm{~kJ} /\left(\mathrm{mol} \cdot \mathrm{nm}^{2}\right)$ was used. This serves to optimize the position of solvent molecules and protein hydrogen atoms.

- We gently start 100 ps of MD simulation in NVT ensemble at $60 \mathrm{~K}$ using Berendsen thermostat with $0.1 \mathrm{ps}$ time constant. Here and further on, we use two separate thermostats for protein and solvent (water + ions) for a more effective temperature control. The protein heavy atoms are still restrained as in minimization run.

- The position restraints on protein heavy atoms are reduced to $1000 \mathrm{~kJ} /\left(\mathrm{mol} \cdot \mathrm{nm}^{2}\right)$ and we begin heating phase from $60 \mathrm{~K}$ to $300 \mathrm{~K}$. The temperature rises linearly for $200 \mathrm{ps}$ and we use larger time constant of 1 ps in Berendsen thermostat. When the final temperature is reached, we continue the simulation for another $100 \mathrm{ps}$.

- We switch to NPT ensemble to obtain proper system density. The thermostat parameters are as in the previous run. The constant pressure of 1 bar is maintained with Berendsen isotropic barostat with 5 ps time constant. We gradually release the restraints on heavy atoms position by performing four simulation runs with force constants of $1000,750,500$ and $250 \mathrm{~kJ} /\left(\mathrm{mol} \cdot \mathrm{nm}^{2}\right)$ each $25 \mathrm{ps}-$ long.

- The final equilibration and production run takes $30 \mathrm{~ns}$ to check for structure stability. It is performed without any restraints on atoms position in NPT ensemble using Nosé-Hoover thermostat and Parrinello-Rahman barostat (time constants equal 5 ps in both cases).

Throughout the classical simulation we use the periodic boundary conditions. The cut-off distance for van der Waals $(\mathrm{vdW})$ and electrostatic interactions was set to $1.1 \mathrm{~nm}$. Long-range electrosatic interactions are treated with smooth particle-mesh Ewald method and the grid density is approximately 1 point per $0.1 \mathrm{~nm}$. The vdW and electrostatic interactions between atoms seperated by 3 covalent bonds (so called 1-4 interactions) are scaled down by a factor of 2 and 1.2 , respectively. We do not use empirical dispersion correction.

\section{S1.2 Hybrid MD simulations}

Starting from an arbitrary structure taken from the last step of the classical MD simulation, we perform QM/MM simulation using Born-Oppenheimer ansatz for QM subsystem in four stages:

- The velocities are generated according to Maxwell distribution at $200 \mathrm{~K}$. A 300 fs long MD run is performed which is enough to reach the target temperature of $200 \mathrm{~K}$ with the canonical sampling through velocity rescaling thermostat and time constant of $10 \mathrm{fs}$. As in classical MD simulation, we use two separate thermostats for protein and the rest of the system. Then, two more stages of $300 \mathrm{fs}$ long heating are performed with the target temperature set to $250 \mathrm{~K}$ and $300 \mathrm{~K}$.

- We continue with 10 ps long simulation using a chain of four Nosé-Hoover thermostats with time constant of 10 fs. All other parameters of the simulations are the same as in previous stages.

The hybrid MD simulation parameters regarding the MM subsystem are as in the classical MD simulation. However, in the original Amber ff99SB*-ildn force field, the vdW parameters of hydrogen atoms in water and hydroxyl groups of serine, threonine and tyrosine amino acid (a.a.) residues are neglected. As a consequence, these atoms belonging to the MM subsystem may come too close to the QM subsystem due to underestimated repulsion at short distance and lead to artifacts. To prevent it, we assign to the named hydrogen atoms the GAFF2 parameters for alcohol hydroxyl group hydrogen atom. The well depth is $0.0047 \mathrm{kcal} / \mathrm{mol}$ and atomic radius is $0.3019 \AA$.

As we indicated in the main text, we use DFT/BLYP/6-31G* level of theory to describe the QM subsytem electronic structure. The Gaussian augmented plane wave (GAPW) [1] scheme was used together with all-electron pseudopotentials for the nuclei. The QM/MM electrostatic coupling is implemented through multigrid algorithm with 4 commensurate grids [2]. The cut-off for the finest grid is set to $400 \mathrm{Ry}$ and Gaussian functions are mapped at 60 Ry (REL_CUTOFF option in $\mathrm{CP} 2 \mathrm{~K}$ ). To prevent the overpolarization of QM cluster electron density, the point charge on the MM atom at the QM/MM boundary is set to zero and is evenly redistributed to the rest of the residue atoms. Since the sum of point charges of a.a. side chain atoms is not zero in Amber force fields, the excessive charge due to including the atoms in QM subsystem is also redistributed evenly into atoms of that a.a. residue remaining in MM subsystem. 


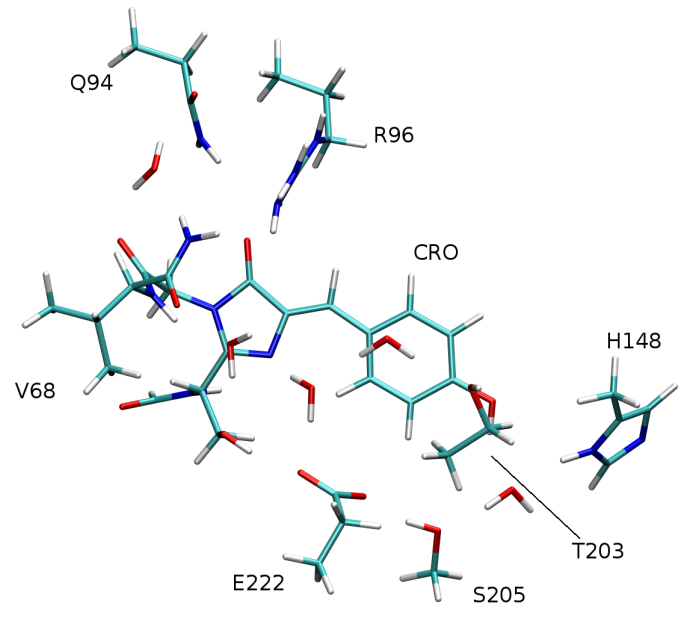

(a) avGFP-n

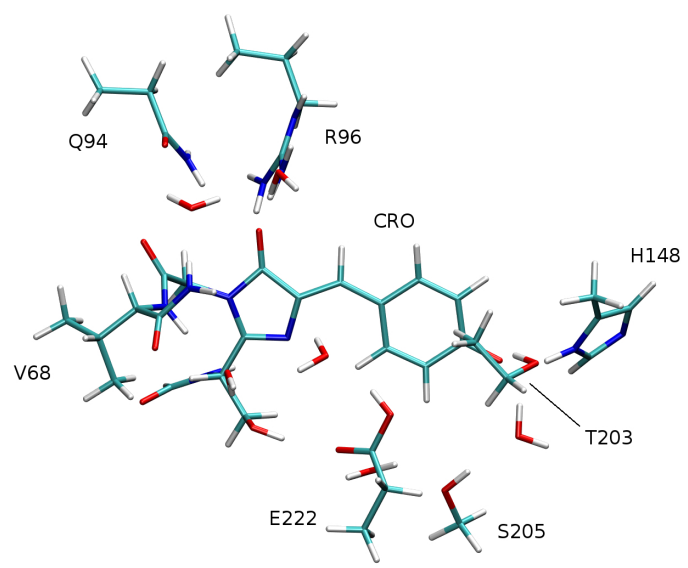

(b) avGFP-a

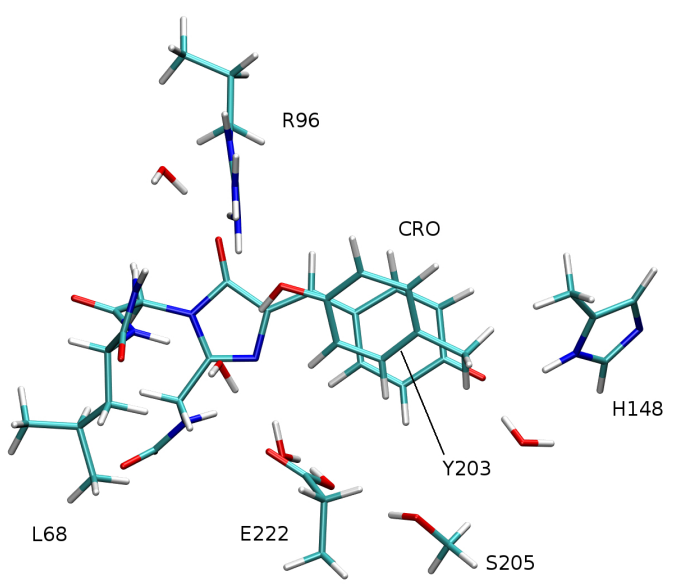

(c) Citrine

Figure S1: The composition QM clusters in QM/MM MD simulations. CRO denotes the chromophore.

\section{S2 FP models structure}

To obtain a more realistic structure of FP in solution, we relaxed the crystallographic structure with classical and hybrid MD simulations. The crystal (with added hydrogen atoms and solvent) and QM/MM MD structures of FPs are available in Gromos87 format files in Supporting Information. In general, the relaxed FP structure that we use for OPA and TPA properties calculations is close to the crystal one. The root mean square deviation (RMSD) of protein excluding hydrogen atoms and mainchain is respectively: $1.57 \AA$ and $1.09 \AA$ (avGFP-n), $0.81 \AA$ and $0.66 \AA$ (avGFP-a), $1.56 \AA$ and $1.00 \AA$ A (Citrine).

We focus on the main structural differences between relaxed and crystal structure near the chromophore. Although we utilize structures from hybrid MD simulation for OPA and TPA spectra calculations, the QM/MM MD simulation is too short to observe significant structural changes. That is why in following text we focus on the results from classical MD run. According to Figure S2a,b, the Q69 residue is virtually parallel to the chromophore long axis in both avGFP-n and avGFP-a crystals. However, upon relaxation, the amide group travels ca. $1.8 \AA$ (this distance changes in range of ca. 0.5-2.25 $\AA$ during classical MD simulation) away from the chromophore. For avGFP-n, this is accompanied by V150 rotation and a significant movement of $\mathrm{W} 1$ and appearence of $\mathrm{W} 2$ water molecules. The latter arrives from the bulk water after about $3.3 \mathrm{~ns}$ of classical MD simulation and is not present in X-ray structure. This leads to pretty stable h-bonds network (Q69-W1-W2-W3). This is slightly different for avGFP-a (Figure S2b). The nearby water molecules do not change their position significantly. Still however Q69 is clearly h-bonded to W2 water molecule, and W2 to W1. The W3 water molecule h-bonded to the imidazolinone and Q69 amide oxygen atoms is also marked in Figure S2b. Water molecule is not present in avGFP-n in a similar position and Q69 still preferentially drifts away from the chromophore, however to a smaller extent than in avGFP-a. The "bridging" water molecule may further favourize pushing the Q69 side chain away from the chromophore. 


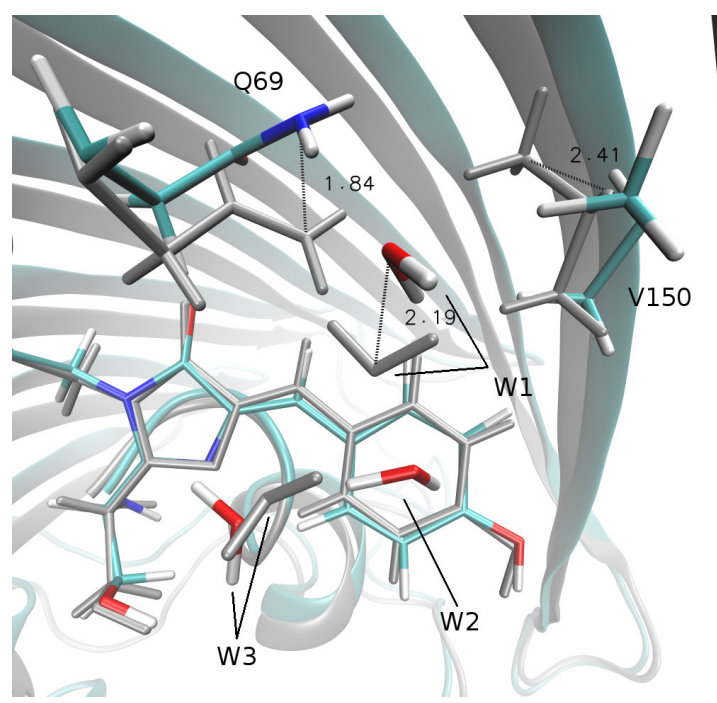

(a) avGFP-n

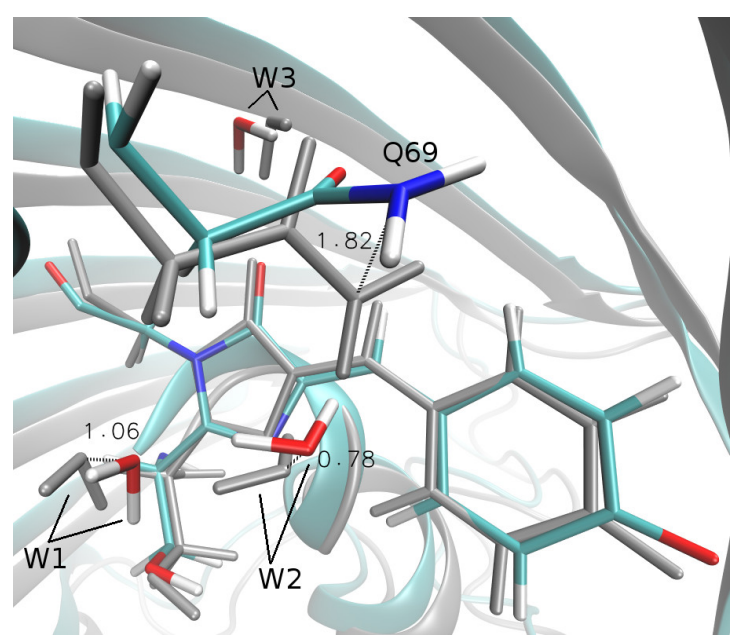

(b) avGFP-a

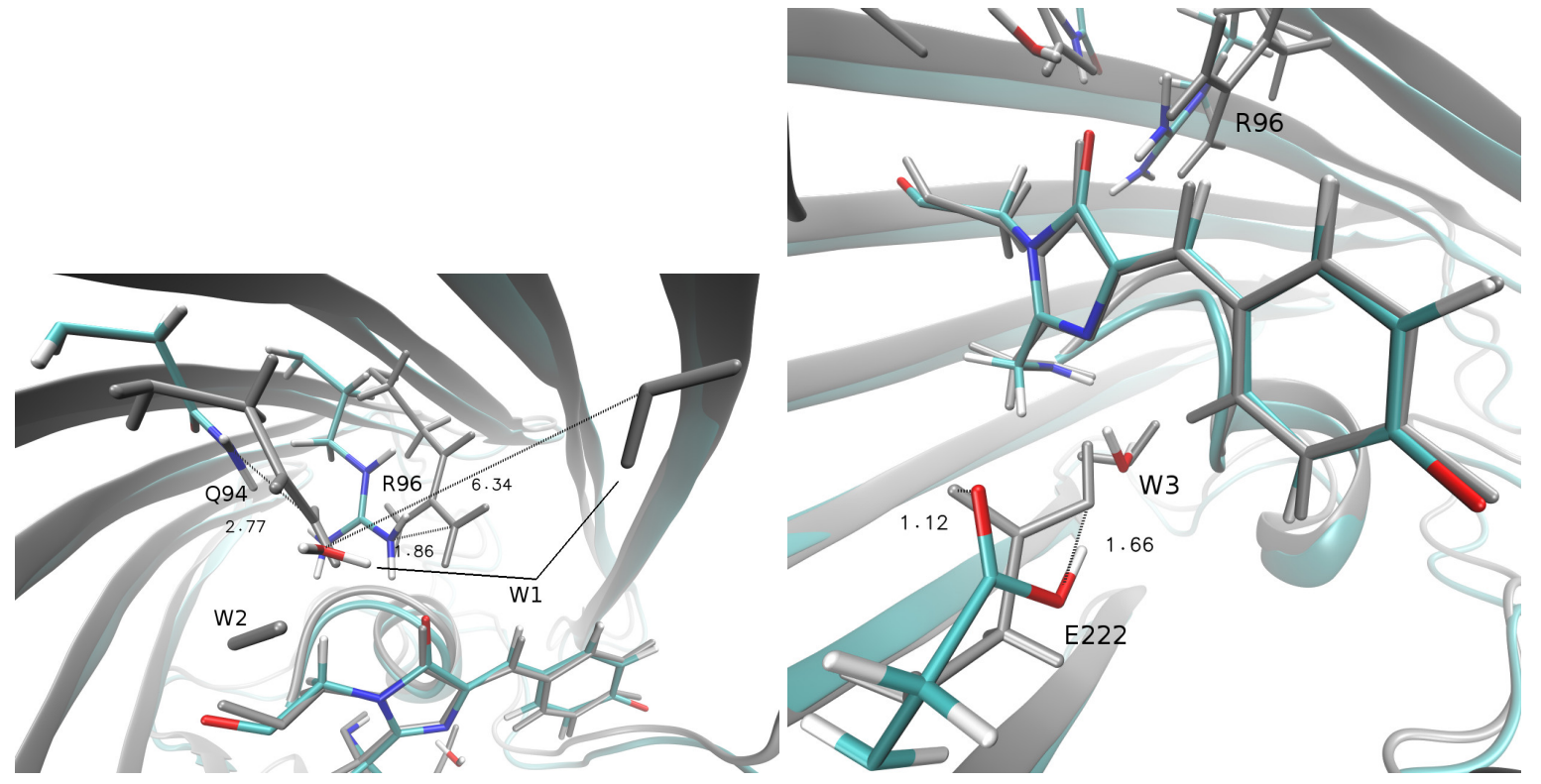

(c) Citrine

Figure S2: The structural differences in the chromophore vicinity between crystallographic (grey) and hybrid MD relaxed structures (coloured according to atom names). For chosen atoms, distance traveled in ångstroms during relaxation is given. The structures were overlapped so that the RMSD between chromophore atoms is minimum. Hydrogen in white, carbon in cyan, oxygen in red, nitrogen in blue.

In case of Citrine, we observe much more extensive changes in the chromophore's environment as compared to the crystal structure. According to Figure S2c, the Q94 residue is h-bonded to chromophore in crystal. Just before all restraints on heavy protein atoms were released in classical MD simulation, we observe two water molecules including W1 on the opposite site of the imidazolinone oxygen (not shown in Figure S2c). They initially push the Q94 side chain away from chromophore and then one of them drifts away, but W1 stays and creates a strong h-bond with the imidazolinone. At this moment, Q94 is not able to reestablish the h-bond with the chromophore (even after we extended the MD simulation to $30 \mathrm{~ns}$ ). As a consequence: i) Q94 drifts away from the chromophore, ii) the R96 side chain partially fills in the gap after Q94. Please note, the W1 water molecule travels a long distance of $6.34 \AA$ from its original position. Most of this distance is covered during heating phase. It is replaced only after 9 ns of classical MD simulation by another crystal water molecule. This shows a high mobility of water molecules inside $\beta$-barrel. It is not suprising and was also observed in other FPs, including avGFP-n and avGFP-a. It is important to inform the reader that W1 water molecule is not present in crystal structure but it fills a cavity inside Citrine after adding the solvent when we built the system. That may mean, this this is not an optimal position for that water molecule. On the other hand, even if we delete two water molecules 
that initialize Q94 movement, we still observe that Q94-chromophore h-bond is broken this time due to another water molecule appearence in position of W1. Plausibly, the result we obtain is correct for that system. The 1 HUY crystal structure resolution is $2.2 \AA$ and maybe these water molecules could not be resolved, although water molecule is also not predicted in this position by higher-resolution (1.59 $\AA$ ) 3DPW structure [3]. Furthermore, we observe one more important structural rearrangement in Citrine. Namely, the E222 is h-bonded (h-bond length is $3.14 \AA$ ) to one of imidazolinone nitrogen atoms according to X-ray structure. However, upon MD relaxation, the protonated oxygen atom moves away from the chromophore by $1.66 \AA$. The E222-chromophore h-bond is now mediated by W3 water molecule which virtually does not change its position relative to crystal structure. It is possible that this is more stable construct than a direct E222chromophore interaction. We find the latter however after almost $23 \mathrm{~ns}$ of classical MD simulation.

The observed structural rearrangements could be attributed to the artifacts stemming from the system structure model (some water molecules fill in gaps inside the protein when the solvent is added) or the interactions model (force field). However, recently Boxer and co-workers [4] reported that for a mutant of avGFP-a, the conformation of a.a. residues facing the chromophore may strongly depend on the crystallization conditions, "suggesting that crystal structures should be interpreted with care" [4]. They conclude that the consequence of different chromophore's environment is a different photoisomerization mechanism. Analogously, the OPA and TPA spectra - usually reported in solution, not crystal will also be strongly influenced by conformation of a.a. residues and position of water molecules especially in the chromophore's immediate environment. The crystal field produced by ordered proteins is a powerful factor in determining the conformation of a.a. residues. We thus believe that our relaxed structures are still reliable representation of investigated FPs considering Boxer's findings [4]. 


\section{S3 Composition of QM subsystems}

Table S1: Size and composition of QM clusters for OPA and TPA properties calculations for the avGFP-n. See also Tables S2 and S3.

\begin{tabular}{|c|c|c|}
\hline Name & Size & Composition \\
\hline 0.00 & 40 & Chromophore only \\
\hline 0.20 & 56 & $0.00+\mathrm{E} 222+2 \mathrm{xH}_{2} \mathrm{O}$ \\
\hline int1 & 69 & $0.00+\mathrm{R} 96+\mathrm{E} 222$ \\
\hline int2 & 84 & int $1+5 \mathrm{xH}_{2} \mathrm{O}$ \\
\hline int3 & 117 & int2 + Q94 + N121 + H148 \\
\hline 0.25 & 140 & $0.20+\mathrm{T} 62+\mathrm{Q} 94+\mathrm{R} 96+\mathrm{N} 121+\mathrm{H} 148+\mathrm{L} 220+3 \mathrm{xH}_{2} \mathrm{O}$ \\
\hline $0.30-$ noh $^{[a]}$ & 141 & int $3+\mathrm{T} 62+\mathrm{S} 205+3 \mathrm{xH}_{2} \mathrm{O}$ \\
\hline int4 & 144 & int3 + Q69 + T203 + S205 \\
\hline $0.35-$ noh $^{[b]}$ & 178 & int $4+\mathrm{T} 62+\mathrm{Y} 145+3 \mathrm{xH}_{2} \mathrm{O}$ \\
\hline int5 & 205 & $\begin{array}{l}0.35-\mathrm{noh}+\mathrm{L} 44-\mathrm{Q} 69+\mathrm{V} 112-\mathrm{Y} 145+\mathrm{V} 150+\mathrm{I} 167-\mathrm{T} 203+\mathrm{L} 220 \\
\text { or int4 + L44 + T62 - Q69 + V112 + V150 + I167 - T203 + L220 + 3xH } 2 \mathrm{O}\end{array}$ \\
\hline int6 & 208 & 0.35-noh - N121 + V150 + I167 + L220 \\
\hline int7 & 231 & int6 + L42 + N121 \\
\hline 0.30 & 234 & $\begin{array}{l}0.25+\mathrm{L} 42+\mathrm{L} 44+\mathrm{V} 112+\mathrm{V} 150+\mathrm{F} 165+\mathrm{I} 167+\mathrm{S} 205+3 \mathrm{xH}_{2} \mathrm{O} \\
\text { or int7 + L44 - Q69 + V112 - Y145 + F165 - T203 }\end{array}$ \\
\hline int8 & 242 & $0.30-\mathrm{L} 42+\mathrm{Q} 69+\mathrm{Y} 145-\mathrm{F} 165+\mathrm{T} 203$ \\
\hline int9 & 256 & int7 + L44 + V112 \\
\hline int10 & 271 & int $9+$ F165 \\
\hline 0.35 & 286 & $0.30+\mathrm{F} 46+\mathrm{Q} 69+\mathrm{Y} 145+\mathrm{T} 203$ \\
\hline 0.40 & 300 & $0.35+\mathrm{V} 61+1 \mathrm{xH}_{2} \mathrm{O}$ \\
\hline 0.45 & 326 & $0.40+\mathrm{T} 63+\mathrm{I} 123+1 \mathrm{xH}_{2} \mathrm{O}$ \\
\hline 0.50 & 365 & $0.45+\mathrm{Y} 92+\mathrm{Q} 183+\mathrm{V} 224$ \\
\hline
\end{tabular}

[a] - all a.a. residues and water molecules within $0.30 \mathrm{~nm}$ radius but excluding hydrophobic residues.

[b] - all a.a. residues and water molecules within $0.35 \mathrm{~nm}$ radius but excluding hydrophobic residues. 
Table S2: Composition of QM clusters for OPA and TPA properties calculations for the avGFP-n. In bold we refer to amino acid residues that were most frequently found in $0.30 \mathrm{~nm}$ radius from chromophore during last stage of $\mathrm{QM} / \mathrm{MM} \mathrm{MD}$ simulation.

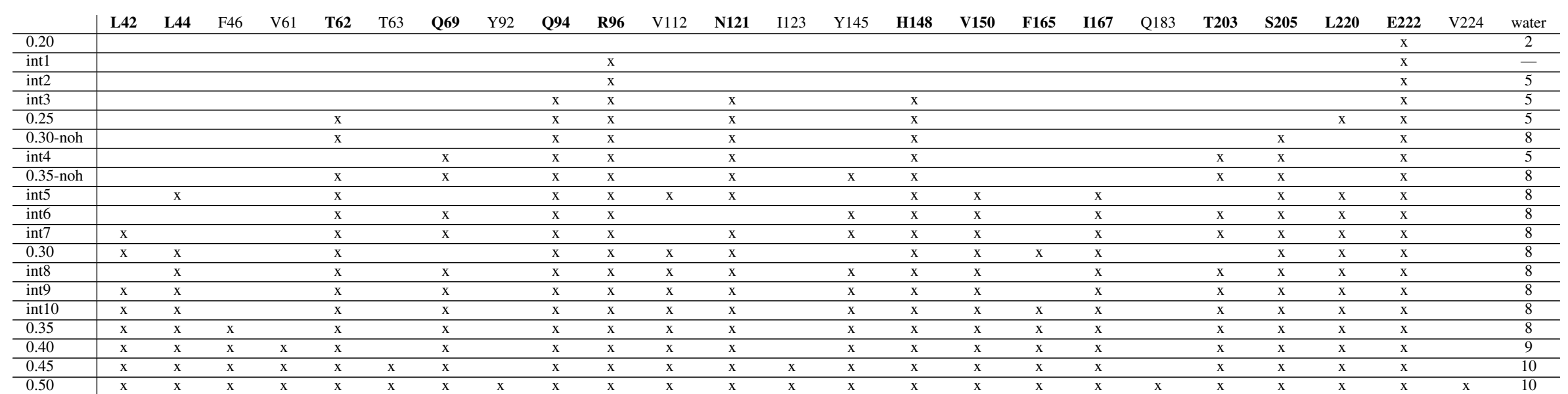


Table S3: Water molecules included in the QM clusters of avGFP-n. Their placement is shown below. In bold we refer to water molecules that were most frequently found in $0.30 \mathrm{~nm}$ radius from chromophore during last stage of QM/MM MD simulation.

\begin{tabular}{|c|c|c|c|c|c|c|c|c|c|c|}
\hline System & 232 & 236 & 241 & 246 & 248 & 251 & 256 & 3603 & 3615 & 5621 \\
\hline 0.20 & & & $\mathrm{X}$ & & & $\mathrm{x}$ & & & & \\
\hline \multicolumn{11}{|l|}{ int1 } \\
\hline int2 & $\mathrm{x}$ & $\mathrm{x}$ & $\mathrm{x}$ & & & $\mathrm{x}$ & $\mathrm{x}$ & & & \\
\hline int3 & $\mathrm{x}$ & $\mathrm{x}$ & $\mathrm{x}$ & & & $\mathrm{x}$ & $\mathrm{x}$ & & & \\
\hline 0.25 & $\mathrm{x}$ & $\mathrm{x}$ & $\mathrm{x}$ & & & $\mathrm{x}$ & $\mathrm{x}$ & & & \\
\hline 0.30-noh & $\mathrm{x}$ & $\mathrm{x}$ & $\mathrm{x}$ & & & $\mathrm{x}$ & $\mathrm{x}$ & $\mathrm{x}$ & $\mathrm{x}$ & $\mathrm{x}$ \\
\hline int4 & $\mathrm{x}$ & $\mathrm{x}$ & $\mathrm{x}$ & & & $\mathrm{x}$ & $\mathrm{x}$ & & & \\
\hline 0.35-noh & $\mathrm{x}$ & $\mathrm{X}$ & $\mathrm{x}$ & & & $\mathrm{x}$ & $\mathrm{x}$ & $\mathrm{x}$ & $\mathrm{x}$ & $\mathrm{x}$ \\
\hline int5 & $\mathrm{X}$ & $\mathrm{x}$ & $\mathrm{x}$ & & & $\mathrm{x}$ & $\mathrm{x}$ & $\mathrm{x}$ & $\mathrm{x}$ & $\mathrm{x}$ \\
\hline int6 & $\mathrm{x}$ & $\mathrm{x}$ & $x$ & & & $\mathrm{x}$ & $x$ & $x$ & $\mathrm{x}$ & $\mathrm{x}$ \\
\hline int7 & $\mathrm{x}$ & $\mathrm{x}$ & $\mathrm{x}$ & & & $\mathrm{x}$ & $\mathrm{x}$ & $\mathrm{x}$ & $\mathrm{x}$ & $\mathrm{x}$ \\
\hline 0.30 & $\mathrm{x}$ & $\mathrm{x}$ & $\mathrm{x}$ & & & $\mathrm{x}$ & $x$ & $\mathrm{x}$ & $\mathrm{x}$ & $\mathrm{x}$ \\
\hline int8 & $\mathrm{x}$ & $\mathrm{x}$ & $x$ & & & $\mathrm{x}$ & $\mathrm{x}$ & $\mathrm{x}$ & $\mathrm{x}$ & $\mathrm{x}$ \\
\hline int9 & $\mathrm{x}$ & $\mathrm{x}$ & $\mathrm{x}$ & & & $\mathrm{x}$ & $\mathrm{x}$ & $\mathrm{x}$ & $\mathrm{x}$ & $\mathrm{x}$ \\
\hline int10 & $\mathrm{x}$ & $\mathrm{x}$ & $\mathrm{x}$ & & & $\mathrm{x}$ & $\mathrm{x}$ & $\mathrm{x}$ & $\mathrm{x}$ & $\mathrm{x}$ \\
\hline 0.35 & $\mathrm{x}$ & $\mathrm{x}$ & $\mathrm{x}$ & & & $\mathrm{x}$ & $\mathrm{x}$ & $\mathrm{x}$ & $\mathrm{x}$ & $\mathrm{x}$ \\
\hline 0.40 & $\mathrm{x}$ & $\mathrm{x}$ & $x$ & $\mathrm{x}$ & & $x$ & $\mathrm{x}$ & $\mathrm{x}$ & $\mathrm{x}$ & $\mathrm{x}$ \\
\hline 0.45 & $\mathrm{x}$ & $\mathrm{x}$ & $\mathrm{X}$ & $\mathrm{x}$ & $\mathrm{x}$ & $\mathrm{x}$ & $\mathrm{x}$ & $\mathrm{x}$ & $\mathrm{x}$ & $\mathrm{x}$ \\
\hline 0.50 & $\mathrm{x}$ & $\mathrm{X}$ & $\mathrm{x}$ & $\mathrm{x}$ & $\mathrm{X}$ & $\mathrm{x}$ & $\mathrm{x}$ & $\mathrm{x}$ & $\mathrm{x}$ & $\mathrm{x}$ \\
\hline
\end{tabular}

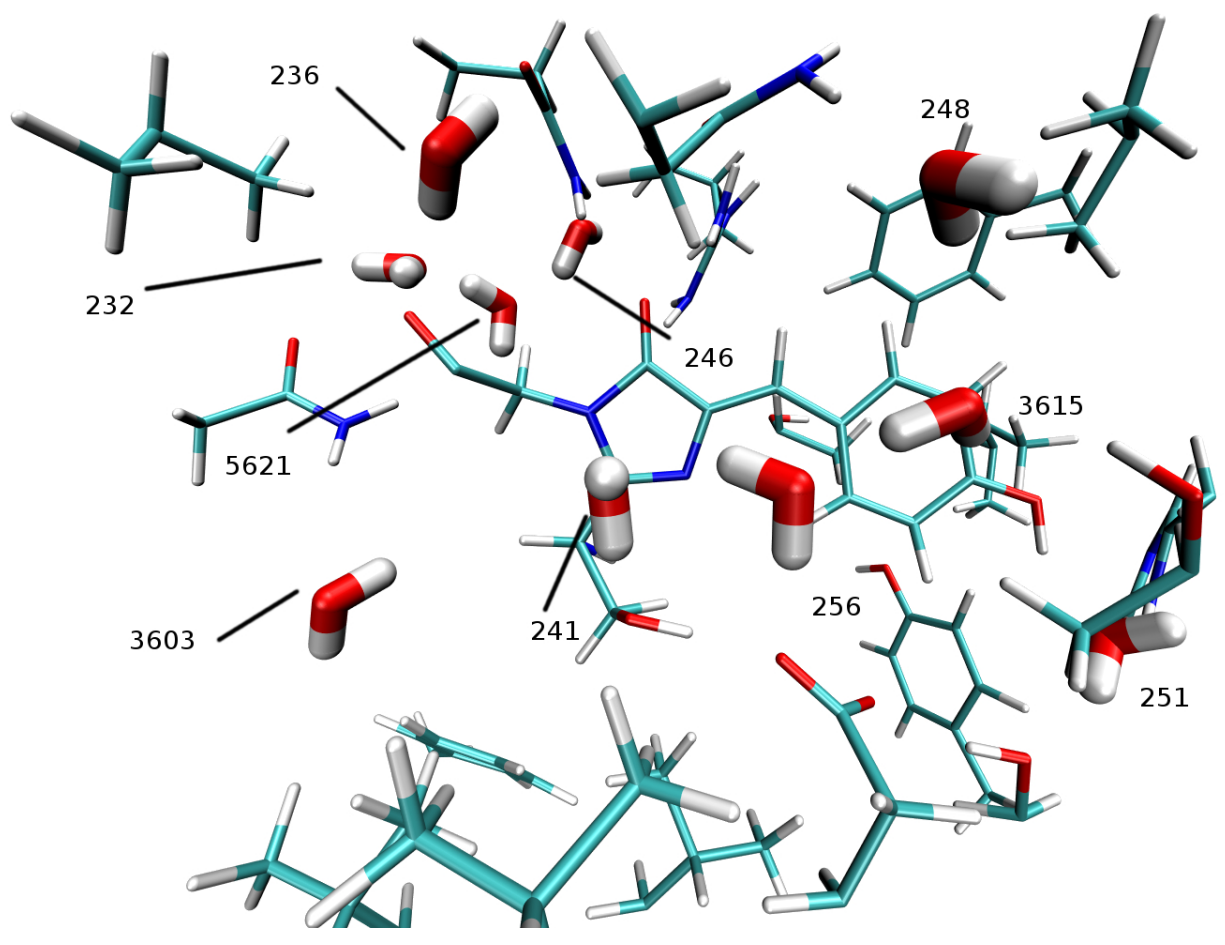


Table S4: Size and composition of QM clusters for OPA and TPA properties calculations for the avGFP-a. See also Tables S5 and S6.

\begin{tabular}{|c|c|c|}
\hline Name & Size & Composition \\
\hline 0.00 & 39 & Chromophore only \\
\hline int1 & 69 & $0.00+\mathrm{R} 96+\mathrm{E} 222$ \\
\hline int2 & 81 & int $1+4 \mathrm{xH}_{2} \mathrm{O}$ \\
\hline 0.20 & 96 & $0.00+\mathrm{R} 96+\mathrm{H} 148+\mathrm{T} 203+\mathrm{E} 222+2 \mathrm{xH}_{2} \mathrm{O}$ \\
\hline int3 & 111 & int $2+\mathrm{T} 62+\mathrm{Q} 94+3 \mathrm{xH}_{2} \mathrm{O}$ \\
\hline 0.25 & 114 & $0.20+\mathrm{Q} 94+2 \mathrm{xH}_{2} \mathrm{O}$ \\
\hline $0.30-\operatorname{noh}^{\lfloor a]}$ & 154 & $0.25+\mathrm{T} 62+\mathrm{Y} 145+\mathrm{S} 205+3 \mathrm{xH}_{2} \mathrm{O}$ \\
\hline int4 & 197 & $0.30-$ noh + Q69 + V150 + I167 + 2xH $\mathrm{xH}_{2} \mathrm{O}$ \\
\hline 0.30 & 219 & $0.25+\mathrm{L} 42+\mathrm{V} 61+\mathrm{T} 62+\mathrm{Y} 145+\mathrm{V} 150+\mathrm{F} 165+\mathrm{S} 205+\mathrm{L} 220+3 \mathrm{xH}_{2} \mathrm{O}$ \\
\hline int5 & 220 & $0.25+\mathrm{L} 42+\mathrm{V} 61+\mathrm{T} 62+\mathrm{Q} 69+\mathrm{N} 121+\mathrm{Y} 145+\mathrm{S} 205+\mathrm{L} 220+5 \mathrm{xH}_{2} \mathrm{O}$ \\
\hline int6 & 225 & $0.25+\mathrm{L} 42+\mathrm{T} 62+\mathrm{Q} 69+\mathrm{Y} 145+\mathrm{V} 150+\mathrm{I} 167+\mathrm{S} 205+\mathrm{L} 220+5 \mathrm{xH}_{2} \mathrm{O}$ \\
\hline int7 & 245 & int6 + V61 + N121 \\
\hline 0.35 & 254 & $0.30+\mathrm{F} 46+\mathrm{I} 167+2 \mathrm{xH}_{2} \mathrm{O}$ \\
\hline int8 & 254 & int7 + T63 \\
\hline int9 & 260 & int7 + F165 \\
\hline 0.40 & 284 & $0.35+\mathrm{T} 63+\mathrm{Q} 69+\mathrm{N} 121$ \\
\hline 0.45 & 313 & $0.40+\mathrm{V} 112+\mathrm{Q} 183+2 \mathrm{xH}_{2} \mathrm{O}$ \\
\hline 0.50 & 342 & $0.45+\mathrm{I} 123+\mathrm{H} 181+1 \mathrm{xH}_{2} \mathrm{O}$ \\
\hline
\end{tabular}

[a] - all a.a. residues and water molecules within $0.30 \mathrm{~nm}$ radius but excluding hydrophobic residues. 
Table S5: Composition of QM clusters for OPA and TPA properties calculations for the avGFP-a. In bold we refer to amino acid residues that were most frequently found in $0.30 \mathrm{~nm}$ radius from chromophore during last stage of $\mathrm{QM} / \mathrm{MM} \mathrm{MD}$ simulation.

\begin{tabular}{|c|c|c|c|c|c|c|c|c|c|c|c|c|c|c|c|c|c|c|c|c|c|c|c|}
\hline & L42 & F46 & V61 & T62 & T63 & Q69 & Q94 & R96 & V112 & N121 & I123 & Y145 & H148 & V150 & F165 & I167 & H181 & Q183 & T203 & S205 & L220 & E222 & water \\
\hline int1 & & & & & & & & $\mathrm{x}$ & & & & & & & & & & & & & & $\mathrm{x}$ & - \\
\hline int2 & & & & & & & & $\mathrm{x}$ & & & & & & & & & & & & & & $\mathrm{x}$ & 4 \\
\hline 0.20 & & & & & & & & $\mathrm{x}$ & & & & & $\mathrm{x}$ & & & & & & $\mathrm{x}$ & & & $\mathrm{x}$ & 2 \\
\hline int3 & & & & $\mathrm{x}$ & & & $\mathrm{x}$ & $\mathrm{x}$ & & & & & & & & & & & & & & $\mathrm{x}$ & 7 \\
\hline 0.25 & & & & & & & $\mathrm{x}$ & $\mathrm{x}$ & & & & & $\mathrm{x}$ & & & & & & $\mathrm{x}$ & & & $\mathrm{x}$ & 4 \\
\hline 0.30 -noh & & & & $\mathrm{x}$ & & & $\mathrm{x}$ & $\mathrm{x}$ & & & & $\mathrm{x}$ & $\mathrm{x}$ & & & & & & $\mathrm{x}$ & $\mathrm{x}$ & & $\mathrm{x}$ & 7 \\
\hline int4 & & & & $\mathrm{x}$ & & $\mathrm{x}$ & $\mathrm{x}$ & $\mathrm{x}$ & & & & $\mathrm{x}$ & $\mathrm{x}$ & $\mathrm{x}$ & & & & & $\mathrm{x}$ & $\mathrm{x}$ & & $\mathrm{x}$ & 9 \\
\hline 0.30 & $\mathrm{x}$ & & $\mathrm{x}$ & $\mathrm{x}$ & & & $\mathrm{x}$ & $\mathrm{x}$ & & & & $\mathrm{x}$ & $\mathrm{x}$ & $\mathrm{x}$ & $\mathrm{x}$ & & & & $\mathrm{x}$ & $\mathrm{x}$ & $\mathrm{x}$ & $\mathrm{x}$ & 7 \\
\hline int5 & $\mathrm{x}$ & & $\mathrm{x}$ & $\mathrm{x}$ & & $\mathrm{x}$ & $\mathrm{x}$ & $\mathrm{x}$ & & $\mathrm{x}$ & & $\mathrm{x}$ & $\mathrm{x}$ & & & & & & $\mathrm{x}$ & $\mathrm{x}$ & $\mathrm{x}$ & $\mathrm{x}$ & 9 \\
\hline int6 & $\mathrm{x}$ & & & $\mathrm{x}$ & & $\mathrm{x}$ & $\mathrm{x}$ & $\mathrm{x}$ & & & & $\mathrm{x}$ & $\mathrm{x}$ & $\mathrm{x}$ & & $\mathrm{x}$ & & & $\mathrm{x}$ & $\mathrm{x}$ & $\mathrm{x}$ & $\mathrm{x}$ & 9 \\
\hline int7 & $\mathrm{x}$ & & $\mathrm{x}$ & $\mathrm{x}$ & & $\mathrm{x}$ & $x$ & $x$ & & $\mathrm{x}$ & & $\mathrm{x}$ & $\mathrm{x}$ & $\mathrm{x}$ & & $\mathrm{x}$ & & & $\mathrm{x}$ & $\mathrm{x}$ & $\mathrm{x}$ & $\mathrm{x}$ & 9 \\
\hline 0.35 & $\mathrm{x}$ & $\mathrm{x}$ & $\mathrm{x}$ & $\mathrm{x}$ & & & $\mathrm{x}$ & $\mathrm{x}$ & & & & $\mathrm{x}$ & $\mathrm{x}$ & $\mathrm{x}$ & $\mathrm{x}$ & $\mathrm{x}$ & & & $\mathrm{x}$ & $\mathrm{x}$ & $\mathrm{x}$ & $\mathrm{x}$ & 9 \\
\hline int8 & $\mathrm{x}$ & & $x$ & $\mathrm{x}$ & $\mathrm{x}$ & $\mathrm{x}$ & $x$ & $x$ & & $\mathrm{x}$ & & $x$ & $\mathrm{x}$ & $\mathrm{x}$ & & $\mathrm{x}$ & & & $\mathrm{x}$ & $\mathrm{x}$ & $\mathrm{x}$ & $\mathrm{x}$ & 9 \\
\hline int9 & $\mathrm{x}$ & & $\mathrm{x}$ & $\mathrm{x}$ & & $\mathrm{x}$ & $\mathrm{x}$ & $\mathrm{x}$ & & $\mathrm{x}$ & & $\mathrm{x}$ & $\mathrm{x}$ & $\mathrm{x}$ & $\mathrm{x}$ & $\mathrm{x}$ & & & $\mathrm{x}$ & $\mathrm{x}$ & $\mathrm{x}$ & $\mathrm{x}$ & 9 \\
\hline 0.40 & $\mathrm{x}$ & $\mathrm{x}$ & $x$ & $\mathrm{x}$ & $\mathrm{x}$ & $x$ & $x$ & $x$ & & $x$ & & $x$ & $\mathrm{x}$ & $x$ & $\mathrm{x}$ & $\mathrm{x}$ & & & $\mathrm{x}$ & $\mathrm{x}$ & $\mathrm{x}$ & $\mathrm{x}$ & 9 \\
\hline 0.45 & $\mathrm{x}$ & $\mathrm{x}$ & $\mathrm{x}$ & $\mathrm{x}$ & $\mathrm{x}$ & $\mathrm{x}$ & $\mathrm{x}$ & $\mathrm{x}$ & $\mathrm{x}$ & $\mathrm{x}$ & & $\mathrm{x}$ & $\mathrm{x}$ & $\mathrm{x}$ & $\mathrm{x}$ & $x$ & & $\mathrm{x}$ & $\mathrm{x}$ & $\mathrm{x}$ & $\mathrm{x}$ & $\mathrm{x}$ & 11 \\
\hline 0.50 & $\mathrm{x}$ & $\mathrm{x}$ & $\mathrm{x}$ & $\mathrm{x}$ & $\mathrm{x}$ & $\mathrm{x}$ & $\mathrm{x}$ & $\mathrm{x}$ & $\mathrm{x}$ & $\mathrm{x}$ & $\mathrm{x}$ & $\mathrm{x}$ & $\mathrm{x}$ & $\mathrm{x}$ & $\mathrm{x}$ & $\mathrm{x}$ & $\mathrm{x}$ & $\mathrm{x}$ & $\mathrm{x}$ & $\mathrm{x}$ & $\mathrm{x}$ & $\mathrm{x}$ & 12 \\
\hline
\end{tabular}


Table S6: Water molecules included in following QM clusters of avGFP-a. Their placement is shown below. In bold we refer to water molecules that were most frequently found in $0.30 \mathrm{~nm}$ radius from the chromophore during last stage of QM/MM MD simulation.

\begin{tabular}{|c|c|c|c|c|c|c|c|c|c|c|c|c|}
\hline System & 245 & 269 & 270 & 271 & 294 & 304 & 314 & 3528 & 3543 & 5449 & 5451 & 5452 \\
\hline int2 & $\mathrm{X}$ & & & & & & $\mathrm{X}$ & & $\mathrm{X}$ & & & $\mathrm{X}$ \\
\hline 0.20 & & & & & & & $\mathrm{X}$ & & $\mathrm{X}$ & & & \\
\hline int3 & $\mathrm{x}$ & & $\mathrm{x}$ & & $\mathrm{x}$ & & $\mathrm{x}$ & & $\mathrm{X}$ & & $\mathrm{x}$ & $\mathrm{x}$ \\
\hline 0.25 & $\mathrm{X}$ & & & & & & $\mathrm{X}$ & & $\mathrm{X}$ & & & $\mathrm{x}$ \\
\hline 0.30-noh & $\mathrm{X}$ & & $\mathrm{x}$ & & $\mathrm{X}$ & & $\mathrm{X}$ & & $\mathrm{X}$ & & $\mathrm{x}$ & $\mathrm{x}$ \\
\hline int4 & $\mathrm{X}$ & & $\mathrm{x}$ & $\mathrm{x}$ & $\mathrm{X}$ & & $\mathrm{X}$ & $\mathrm{x}$ & $\mathrm{X}$ & & $\mathrm{X}$ & $\mathrm{x}$ \\
\hline 0.30 & $\mathrm{x}$ & & $\mathrm{x}$ & & $\mathrm{x}$ & & $\mathrm{x}$ & & $\mathrm{x}$ & & $\mathrm{x}$ & $\mathrm{x}$ \\
\hline int5 & $\mathrm{X}$ & & $\mathrm{x}$ & $\mathrm{x}$ & $\mathrm{X}$ & & $\mathrm{X}$ & $\mathrm{x}$ & $\mathrm{X}$ & & $\mathrm{X}$ & $\mathrm{x}$ \\
\hline int6 & $\mathrm{x}$ & & $\mathrm{x}$ & $\mathrm{x}$ & $\mathrm{X}$ & & $\mathrm{x}$ & $\mathrm{x}$ & $\mathrm{X}$ & & $\mathrm{X}$ & $\mathrm{X}$ \\
\hline int7 & $\mathrm{x}$ & & $\mathrm{x}$ & $\mathrm{X}$ & $\mathrm{X}$ & & $\mathrm{X}$ & $\mathrm{x}$ & $\mathrm{X}$ & & $\mathrm{X}$ & $\mathrm{x}$ \\
\hline 0.35 & $\mathrm{X}$ & & $\mathrm{X}$ & $\mathrm{X}$ & $\mathrm{X}$ & & $\mathrm{X}$ & $\mathrm{X}$ & $\mathrm{X}$ & & $\mathrm{X}$ & $\mathrm{x}$ \\
\hline int8 & $\mathrm{x}$ & & $\mathrm{X}$ & $\mathrm{x}$ & $\mathrm{X}$ & & $\mathrm{x}$ & $\mathrm{x}$ & $\mathrm{X}$ & & $\mathrm{X}$ & $\mathrm{X}$ \\
\hline int9 & $\mathrm{x}$ & & $\mathrm{x}$ & $\mathrm{x}$ & $\mathrm{x}$ & & $\mathrm{x}$ & $\mathrm{x}$ & $\mathrm{x}$ & & $\mathrm{x}$ & $\mathrm{x}$ \\
\hline 0.40 & $\mathrm{X}$ & & $\mathrm{X}$ & $\mathrm{x}$ & $\mathrm{X}$ & & $\mathrm{X}$ & $\mathrm{x}$ & $\mathrm{x}$ & & $\mathrm{X}$ & $\mathrm{x}$ \\
\hline 0.45 & $\mathrm{x}$ & $\mathrm{X}$ & $\mathrm{x}$ & $\mathrm{x}$ & $\mathrm{x}$ & $\mathrm{x}$ & $\mathrm{x}$ & $\mathrm{x}$ & $\mathrm{x}$ & & $\mathrm{X}$ & $\mathrm{x}$ \\
\hline 0.50 & $\mathrm{x}$ & $\mathrm{x}$ & $\mathrm{x}$ & $\mathrm{x}$ & $\mathrm{X}$ & $\mathrm{x}$ & $\mathrm{X}$ & $\mathrm{x}$ & $\mathrm{X}$ & $\mathrm{x}$ & $\mathrm{x}$ & $\mathrm{x}$ \\
\hline
\end{tabular}

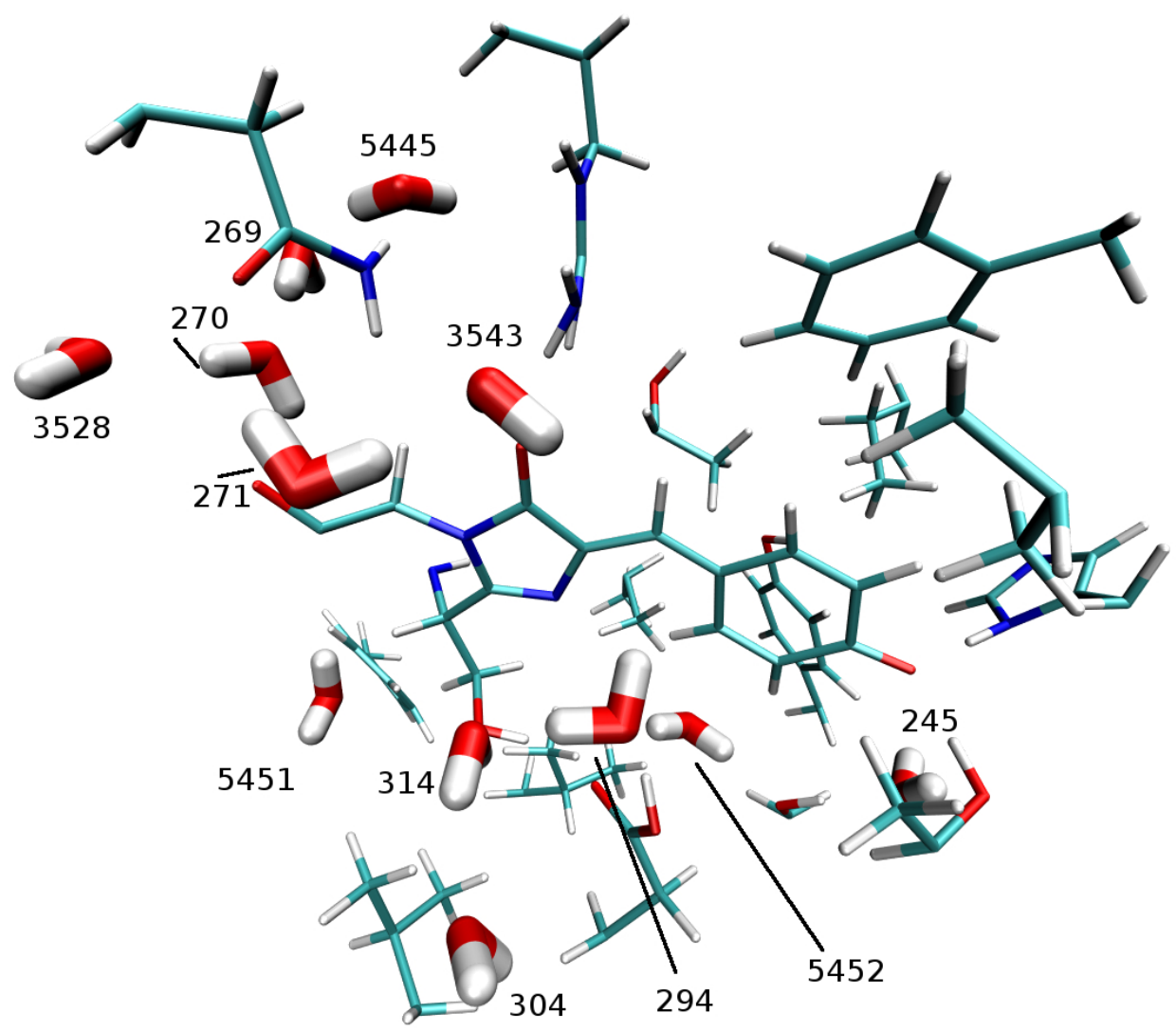


Table S7: Size and composition of QM clusters for OPA and TPA properties calculations of Citrine. See also Tables S8 and $\mathrm{S} 9$.

\begin{tabular}{|c|c|c|}
\hline Name & Size & Composition \\
\hline 0.00 & 35 & Chromophore only \\
\hline int1 & 51 & $0.00+\mathrm{Y} 203$ \\
\hline int2 & 65 & $0.00+\mathrm{R} 96+\mathrm{E} 222$ \\
\hline int3 & 77 & int $2+4 \mathrm{xH}_{2} \mathrm{O}$ \\
\hline 0.20 & 87 & $0.00+\mathrm{M} 69+\mathrm{R} 96+\mathrm{H} 148+3 \mathrm{xH}_{2} \mathrm{O}$ \\
\hline 0.25 & 126 & $0.20+\mathrm{T} 62+\mathrm{Y} 145+\mathrm{V} 150+1 \mathrm{xH}_{2} \mathrm{O}$ \\
\hline int4 & 127 & $0.20-\mathrm{M} 69+\mathrm{Y} 145+\mathrm{Y} 203+\mathrm{S} 205+\mathrm{E} 222+1 \mathrm{xH}_{2} \mathrm{O}$ \\
\hline $0.35-$ noh $^{\lfloor a]}$ & 145 & int $4+\mathrm{T} 62+3 \mathrm{xH}_{2} \mathrm{O}$ \\
\hline int5 & 166 & 0.35-noh + M69 + N121 \\
\hline 0.30 & 167 & $0.25+\mathrm{F} 165+\mathrm{I} 167+\mathrm{S} 205+2 \mathrm{xH}_{2} \mathrm{O}$ \\
\hline int6 & 196 & int5 + V61 - N121 + V150 + I167 + 1xH $\mathrm{xH}_{2} \mathrm{O}$ \\
\hline int7 & 202 & int5 + V112 + V150 + I167 \\
\hline 0.35 & 208 & $0.30+\mathrm{V} 61+\mathrm{Y} 203+\mathrm{E} 222+1 \mathrm{xH}_{2} \mathrm{O}$ \\
\hline int8 & 217 & $0.35+\mathrm{Q} 94+\mathrm{N} 121-\mathrm{F} 165+1 \mathrm{xH}_{2} \mathrm{O}$ \\
\hline 0.40 & 245 & $0.35+\mathrm{L} 44+\mathrm{V} 112+\mathrm{N} 121+1 \mathrm{xH}_{2} \mathrm{O}$ \\
\hline int9 & 246 & int8 + L44 + F165 \\
\hline 0.45 & 301 & $0.40+\mathrm{L} 42+\mathrm{T} 63+\mathrm{Q} 94+\mathrm{Q} 183+3 \times \mathrm{H}_{2} \mathrm{O}$ \\
\hline 0.50 & 348 & $0.45+\mathrm{F} 46+\mathrm{N} 149+\mathrm{L} 220+3 \mathrm{xH}_{2} \mathrm{O}$ \\
\hline
\end{tabular}

[a] - all a.a. residues and water molecules within $0.35 \mathrm{~nm}$ radius but excluding hydrophobic residues. 
Table S8: Composition of QM clusters for OPA and TPA properties calculations for the Citrine. In bold we refer to amino acid residues that were most frequently found in $0.35 \mathrm{~nm}$ radius from chromophore during last stage of $\mathrm{QM} / \mathrm{MM} \mathrm{MD}$ simulation.

\begin{tabular}{|c|c|c|c|c|c|c|c|c|c|c|c|c|c|c|c|c|c|c|c|c|c|c|c|}
\hline & L42 & L44 & F46 & V61 & T62 & T63 & M69 & Q94 & R96 & V112 & N121 & Y145 & H148 & N149 & V150 & F165 & I167 & Q183 & Y203 & S205 & L220 & E222 & water \\
\hline int1 & & & & & & & & & & & & & & & & & & & $\mathrm{x}$ & & & & - \\
\hline int2 & & & & & & & & & $\mathrm{x}$ & & & & & & & & & & & & & $\mathrm{x}$ & - \\
\hline int3 & & & & & & & & & $\mathrm{x}$ & & & & & & & & & & & & & $\mathrm{x}$ & 4 \\
\hline 0.20 & & & & & & & $\mathrm{x}$ & & $\mathrm{x}$ & & & & $\mathrm{x}$ & & & & & & & & & & 3 \\
\hline 0.25 & & & & & $\mathrm{x}$ & & $\mathrm{x}$ & & $\mathrm{x}$ & & & $\mathrm{x}$ & $\mathrm{x}$ & & $\mathrm{x}$ & & & & & & & & 4 \\
\hline int4 & & & & & & & & & $\mathrm{x}$ & & & $\mathrm{x}$ & $\mathrm{x}$ & & & & & & $\mathrm{x}$ & $\mathrm{x}$ & & $\mathrm{x}$ & 4 \\
\hline 0.35 -noh & & & & & $\mathrm{x}$ & & & & $\mathrm{x}$ & & & $\mathrm{x}$ & $\mathrm{x}$ & & & & & & $\mathrm{x}$ & $\mathrm{x}$ & & $\mathrm{x}$ & 7 \\
\hline int5 & & & & & $\mathrm{x}$ & & $\mathrm{x}$ & & $\mathrm{x}$ & & $\mathrm{x}$ & $\mathrm{x}$ & $\mathrm{x}$ & & & & & & $\mathrm{x}$ & $\mathrm{x}$ & & $\mathrm{x}$ & 7 \\
\hline 0.30 & & & & & $\mathrm{x}$ & & $\mathrm{x}$ & & $\mathrm{x}$ & & & $\mathrm{x}$ & $\mathrm{x}$ & & $\mathrm{x}$ & $\mathrm{x}$ & $\mathrm{x}$ & & & $\mathrm{x}$ & & & 6 \\
\hline int6 & & & & $\mathrm{x}$ & $\mathrm{x}$ & & $\mathrm{x}$ & & $\mathrm{x}$ & & & $\mathrm{x}$ & $\mathrm{x}$ & & $\mathrm{x}$ & & $\mathrm{x}$ & & $\mathrm{x}$ & $\mathrm{x}$ & & $\mathrm{x}$ & 8 \\
\hline int7 & & & & & $\mathrm{x}$ & & $\mathrm{x}$ & & $\mathrm{x}$ & $\mathrm{x}$ & $\mathrm{x}$ & $\mathrm{x}$ & $\mathrm{x}$ & & $\mathrm{x}$ & & $\mathrm{x}$ & & $\mathrm{x}$ & $\mathrm{x}$ & & $\mathrm{x}$ & 7 \\
\hline 0.35 & & & & $\mathrm{x}$ & $\mathrm{x}$ & & $\mathrm{x}$ & & $\mathrm{x}$ & & & $\mathrm{x}$ & $\mathrm{x}$ & & $\mathrm{x}$ & $\mathrm{x}$ & $\mathrm{x}$ & & $\mathrm{x}$ & $\mathrm{x}$ & & $\mathrm{x}$ & 7 \\
\hline int8 & & & & $\mathrm{x}$ & $\mathrm{x}$ & & $\mathrm{x}$ & $\mathrm{x}$ & $\mathrm{x}$ & & $\mathrm{x}$ & $\mathrm{x}$ & $\mathrm{x}$ & & $\mathrm{x}$ & & $\mathrm{x}$ & & $\mathrm{x}$ & $\mathrm{x}$ & & $\mathrm{x}$ & 8 \\
\hline 0.40 & & $\mathrm{x}$ & & $\mathrm{x}$ & $\mathrm{x}$ & & $\mathrm{x}$ & & $\mathrm{x}$ & $\mathrm{x}$ & $\mathrm{x}$ & $\mathrm{x}$ & $\mathrm{x}$ & & $\mathrm{x}$ & $\mathrm{x}$ & $\mathrm{x}$ & & $\mathrm{x}$ & $\mathrm{x}$ & & $\mathrm{x}$ & 8 \\
\hline int9 & & $\mathrm{x}$ & & $\mathrm{x}$ & $\mathrm{x}$ & & $\mathrm{x}$ & $\mathrm{x}$ & $\mathrm{x}$ & & $\mathrm{x}$ & $\mathrm{x}$ & $\mathrm{x}$ & & $\mathrm{x}$ & $\mathrm{x}$ & $\mathrm{x}$ & & $\mathrm{x}$ & $\mathrm{x}$ & & $\mathrm{x}$ & 8 \\
\hline 0.45 & $\mathrm{x}$ & $\mathrm{x}$ & & $\mathrm{x}$ & $\mathrm{x}$ & $\mathrm{x}$ & $\mathrm{x}$ & $\mathrm{x}$ & $\mathrm{x}$ & $\mathrm{x}$ & $\mathrm{x}$ & $\mathrm{x}$ & $\mathrm{x}$ & & $\mathrm{x}$ & $\mathrm{x}$ & $\mathrm{x}$ & $\mathrm{x}$ & $\mathrm{x}$ & $\mathrm{x}$ & & $\mathrm{x}$ & 11 \\
\hline 0.50 & $\mathrm{x}$ & $\mathrm{x}$ & $\mathrm{x}$ & $\mathrm{x}$ & $\mathrm{x}$ & $\mathrm{x}$ & $\mathrm{x}$ & $\mathrm{x}$ & $\mathrm{x}$ & $\mathrm{x}$ & $\mathrm{x}$ & $\mathrm{x}$ & $\mathrm{x}$ & $\mathrm{x}$ & $\mathrm{x}$ & $\mathrm{x}$ & $\mathrm{x}$ & $\mathrm{x}$ & $\mathrm{x}$ & $\mathrm{x}$ & $\mathrm{x}$ & $\mathrm{x}$ & 14 \\
\hline
\end{tabular}


Table S9: Water molecules included in following QM clusters of Citrine. Their placement is shown below. In bold we refer to water molecules that were most frequently found in $0.35 \mathrm{~nm}$ radius from the chromophore during last stage of $\mathrm{QM} / \mathrm{MM}$ MD simulation.

\begin{tabular}{|c|c|c|c|c|c|c|c|c|c|c|c|c|c|c|}
\hline System & 231 & 234 & 237 & 239 & 247 & 252 & 337 & 3457 & 3932 & 3935 & 5306 & 5308 & 5318 & 5320 \\
\hline int3 & $\mathrm{X}$ & & & & $\mathrm{x}$ & & & & & $\mathrm{x}$ & & & $\mathrm{X}$ & \\
\hline 0.20 & & & & & $\mathrm{X}$ & & & & & $\mathrm{x}$ & & & $\mathrm{X}$ & \\
\hline 0.25 & $\mathrm{x}$ & & & & $\mathrm{X}$ & & & & & $\mathrm{x}$ & & & $\mathrm{X}$ & \\
\hline int4 & $\mathrm{x}$ & & & & $\mathrm{x}$ & & & & & $\mathrm{x}$ & & & $\mathrm{x}$ & \\
\hline 0.35-noh & $\mathrm{x}$ & & & & $\mathrm{X}$ & $\mathrm{x}$ & & & & $\mathrm{x}$ & $\mathrm{x}$ & & $\mathrm{X}$ & $\mathrm{x}$ \\
\hline int5 & $\mathrm{x}$ & & & & $\mathrm{x}$ & $\mathrm{x}$ & & & & $\mathrm{x}$ & $\mathrm{X}$ & & $\mathrm{x}$ & $\mathrm{x}$ \\
\hline 0.30 & $\mathrm{x}$ & & & & $\mathrm{x}$ & & & & & $\mathrm{x}$ & $\mathrm{x}$ & & $\mathrm{x}$ & $\mathrm{x}$ \\
\hline int6 & $\mathrm{x}$ & & & & $\mathrm{x}$ & $\mathrm{x}$ & & & & $\mathrm{x}$ & $\mathrm{x}$ & $\mathrm{x}$ & $\mathrm{x}$ & $\mathrm{x}$ \\
\hline int7 & $\mathrm{x}$ & & & & $\mathrm{X}$ & $\mathrm{x}$ & & & & $\mathrm{x}$ & $\mathrm{x}$ & & $\mathrm{X}$ & $\mathrm{x}$ \\
\hline 0.35 & $\mathrm{X}$ & & & & $\mathrm{X}$ & $\mathrm{X}$ & & & & $\mathrm{x}$ & $\mathrm{X}$ & & $\mathrm{X}$ & $\mathrm{x}$ \\
\hline int8 & $\mathrm{x}$ & & & & $\mathrm{X}$ & $\mathrm{x}$ & & & & $\mathrm{x}$ & $\mathrm{x}$ & $\mathrm{x}$ & $\mathrm{X}$ & $\mathrm{x}$ \\
\hline 0.40 & $\mathrm{x}$ & & & & $\mathrm{X}$ & $\mathrm{x}$ & & & & $\mathrm{X}$ & $\mathrm{x}$ & $\mathrm{x}$ & $\mathrm{X}$ & $\mathrm{x}$ \\
\hline int9 & $\mathrm{x}$ & & & & $\mathrm{X}$ & $\mathrm{x}$ & & & & $\mathrm{x}$ & $\mathrm{X}$ & $\mathrm{x}$ & $\mathrm{X}$ & $\mathrm{x}$ \\
\hline 0.45 & $\mathrm{x}$ & & & $\mathrm{x}$ & $\mathrm{x}$ & $\mathrm{x}$ & $\mathrm{x}$ & $\mathrm{x}$ & & $\mathrm{x}$ & $\mathrm{x}$ & $\mathrm{x}$ & $\mathrm{x}$ & $\mathrm{x}$ \\
\hline 0.50 & $\mathrm{X}$ & $\mathrm{X}$ & $\mathrm{X}$ & $\mathrm{X}$ & $\mathrm{X}$ & $\mathrm{X}$ & $\mathrm{X}$ & $\mathrm{X}$ & $\mathrm{X}$ & $\mathrm{x}$ & $\mathrm{X}$ & $\mathrm{X}$ & $\mathrm{X}$ & $\mathrm{x}$ \\
\hline
\end{tabular}

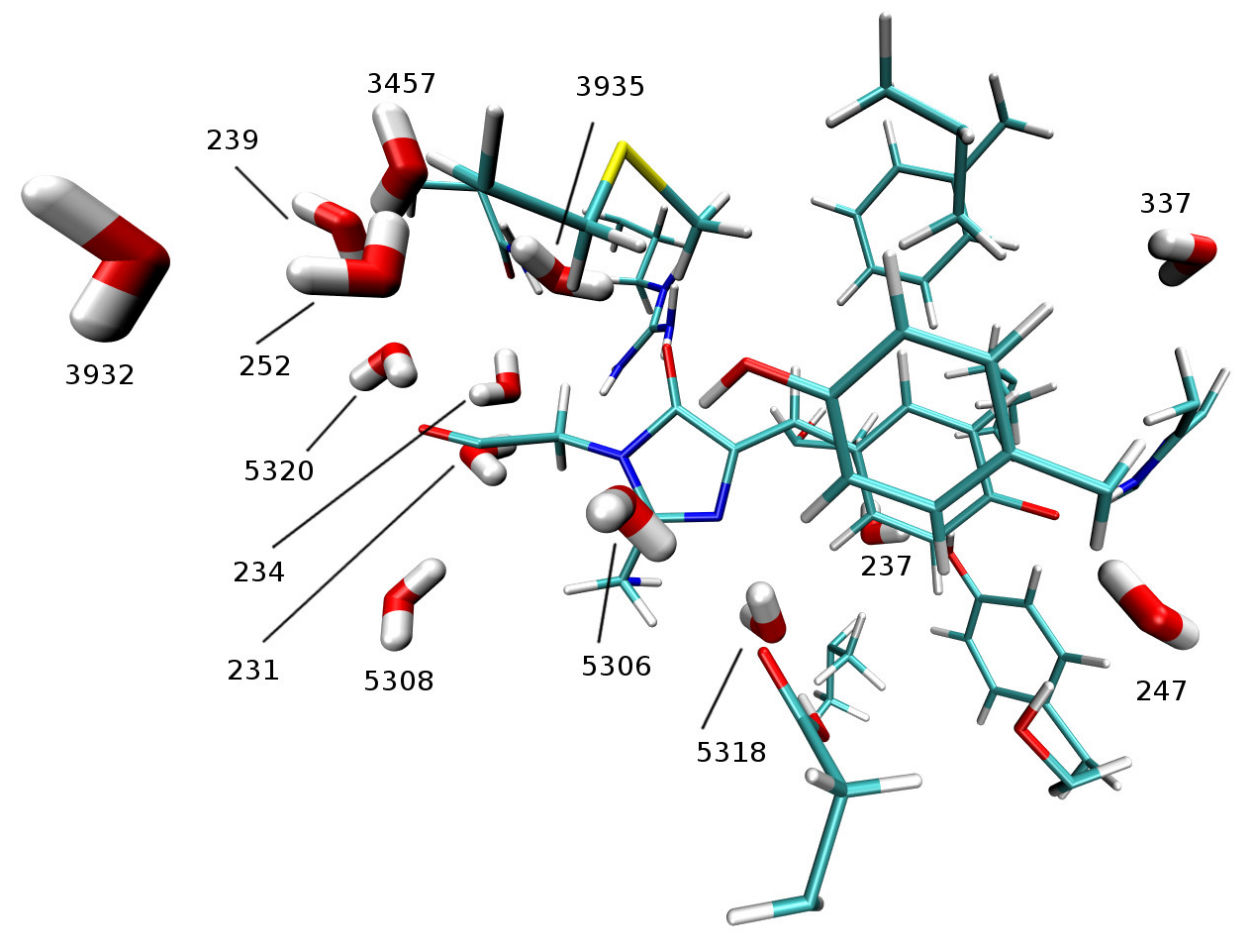




\section{S4 Full set of OPA and TPA spectra}
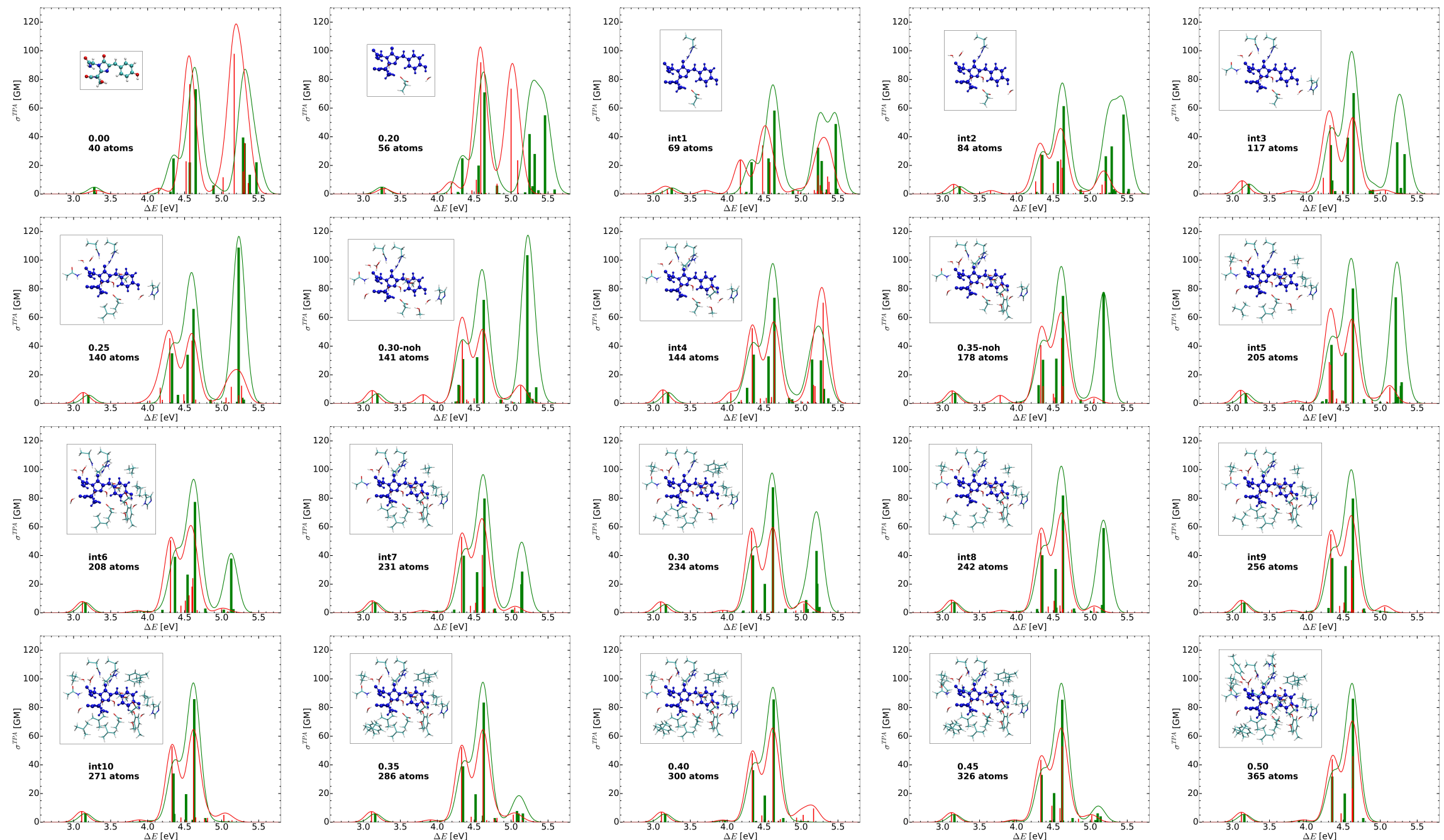

Figure S3: TPA spectra for avGFP-n models. No embedding in red, electrostatic embedding in green. 

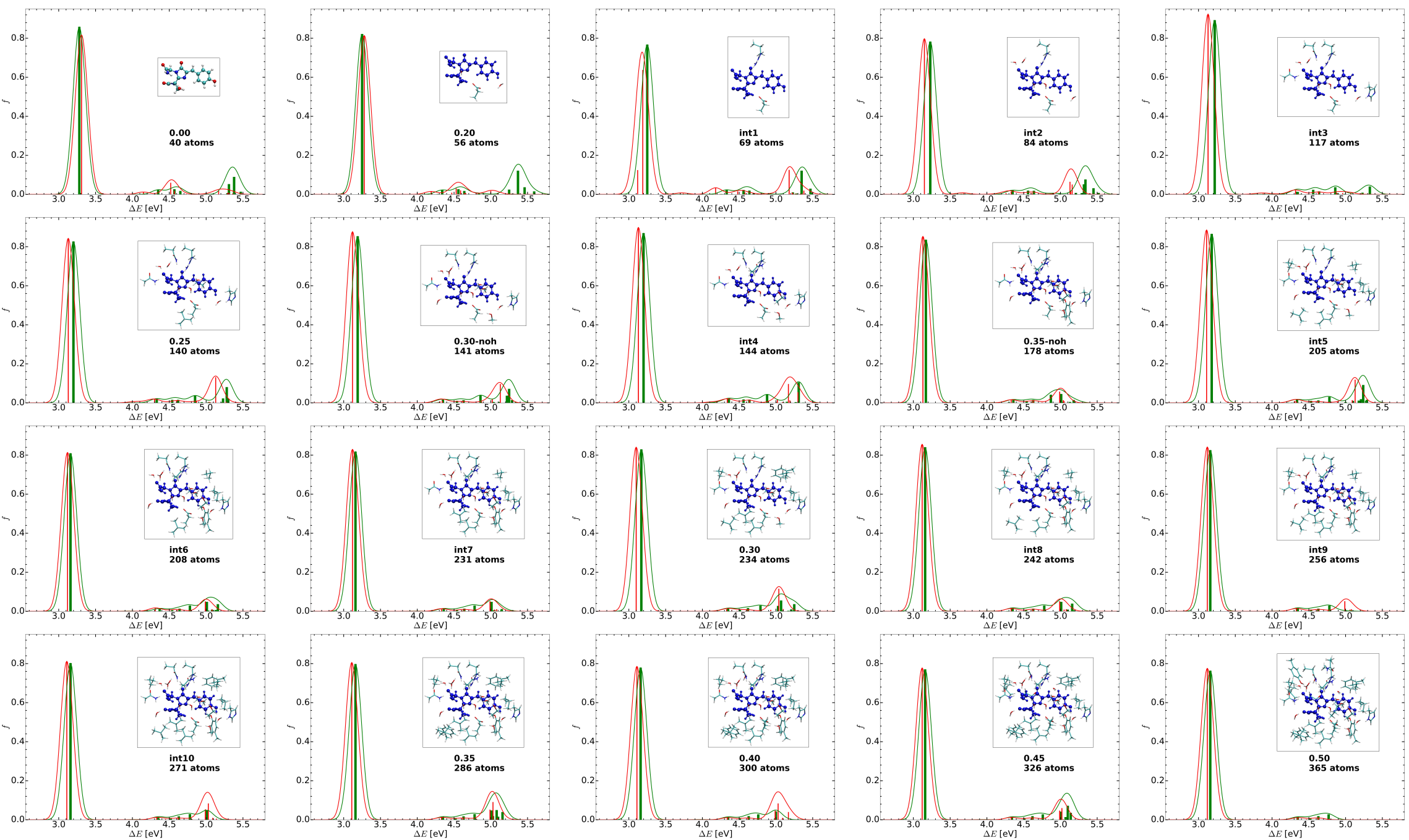

Figure S4: OPA spectra for avGFP-n models. No embedding in red, electrostatic embedding in green. 

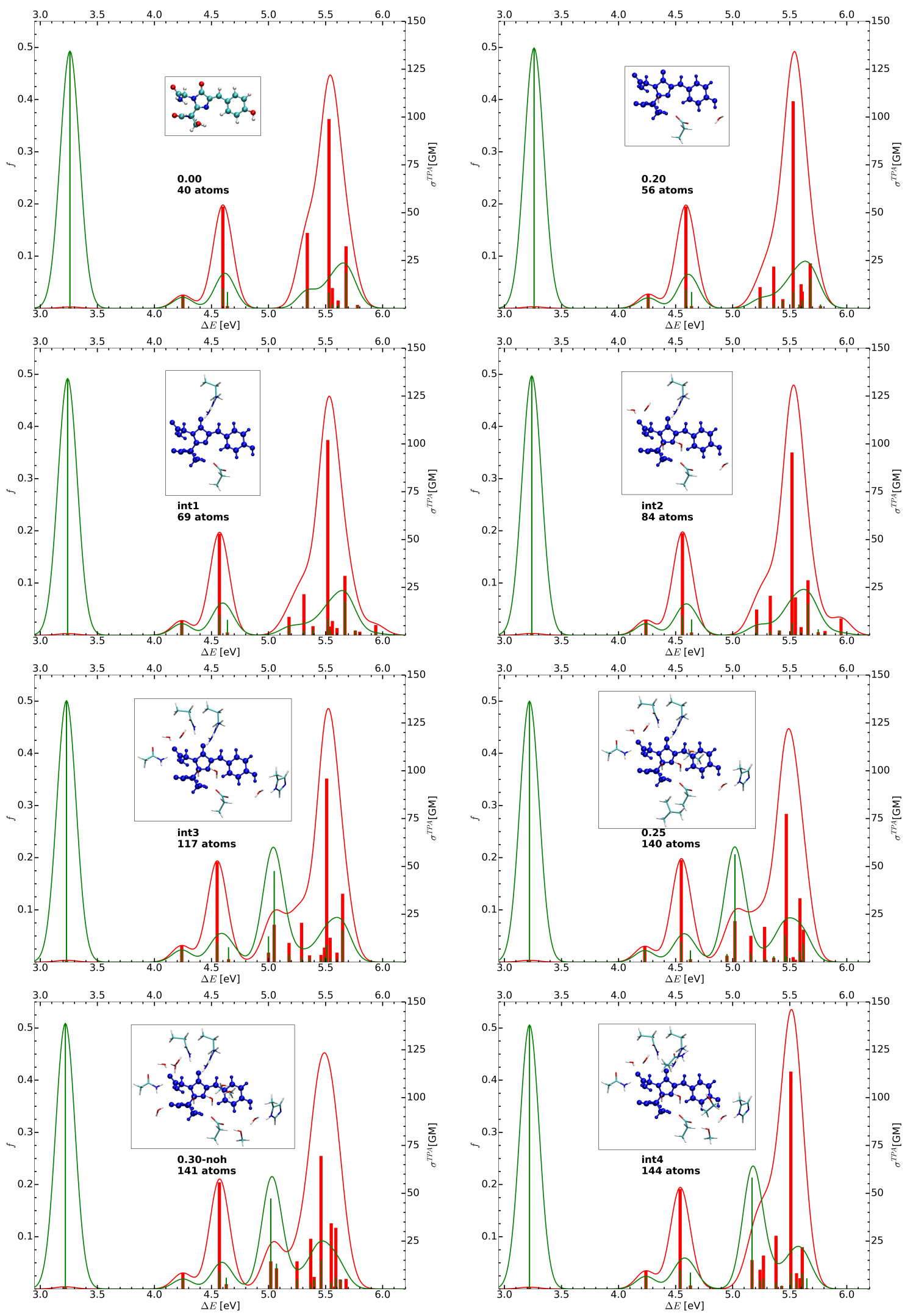

Figure S5: OPA (green) and TPA (red) spectra of avGFP-n models with the polarizable embedding applied. 

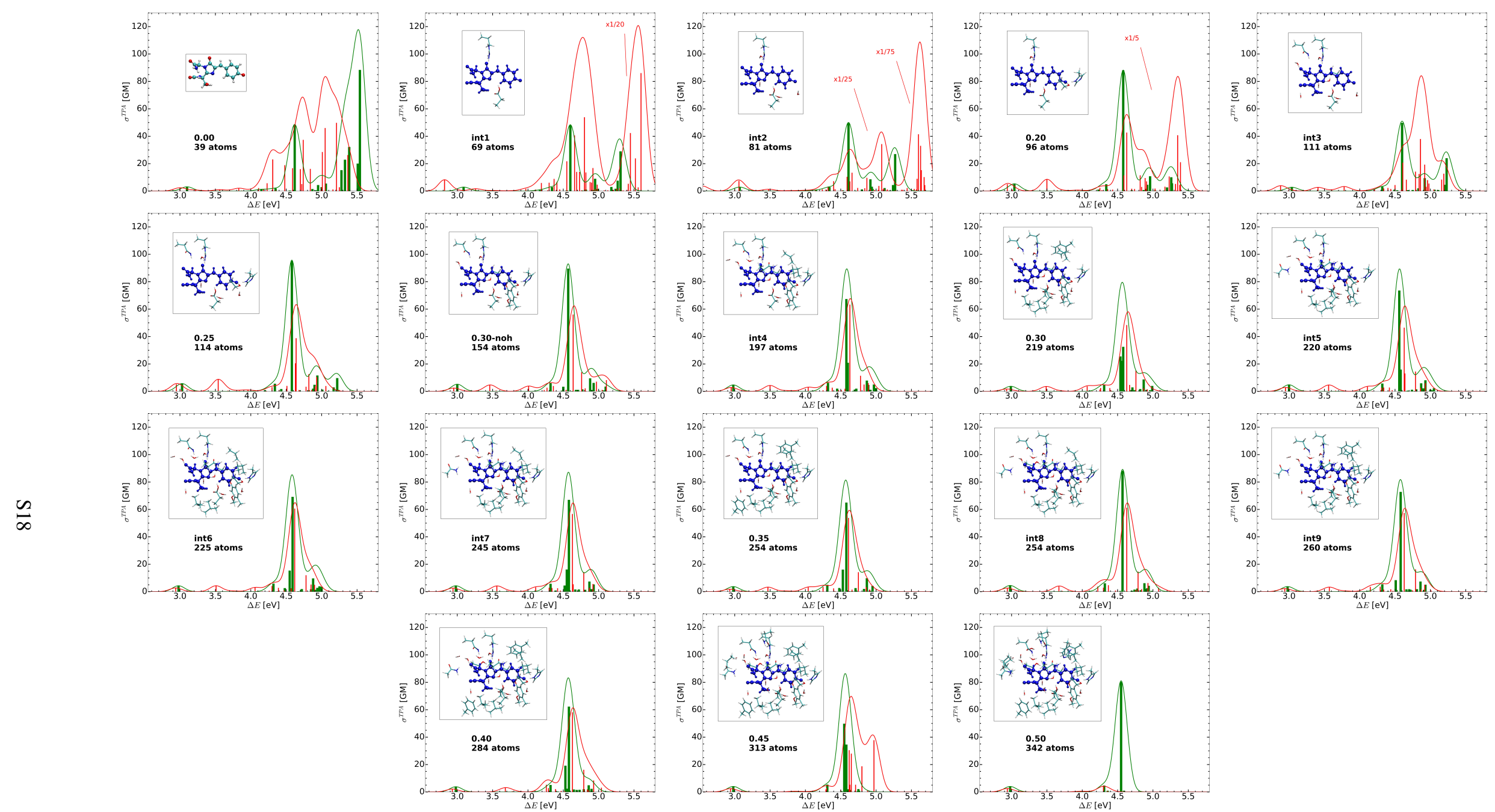

Figure S6: TPA spectra for avGFP-a models. No embedding in red, electrostatic embedding in green. In case of the int1/NE and $0.20 / \mathrm{NE}$ models, we rescaled all $\sigma^{T P A}$ values by a factor given on the plot if $\Delta E>5.0 \mathrm{eV}$. In case of the int2/NE models, we rescaled all $\sigma^{T P A}$ values by $1 / 25$ if $4.9<\Delta E \leq 5.2 \mathrm{eV}$ and by $1 / 75$ if $\Delta E>5.2 \mathrm{eV}$. 

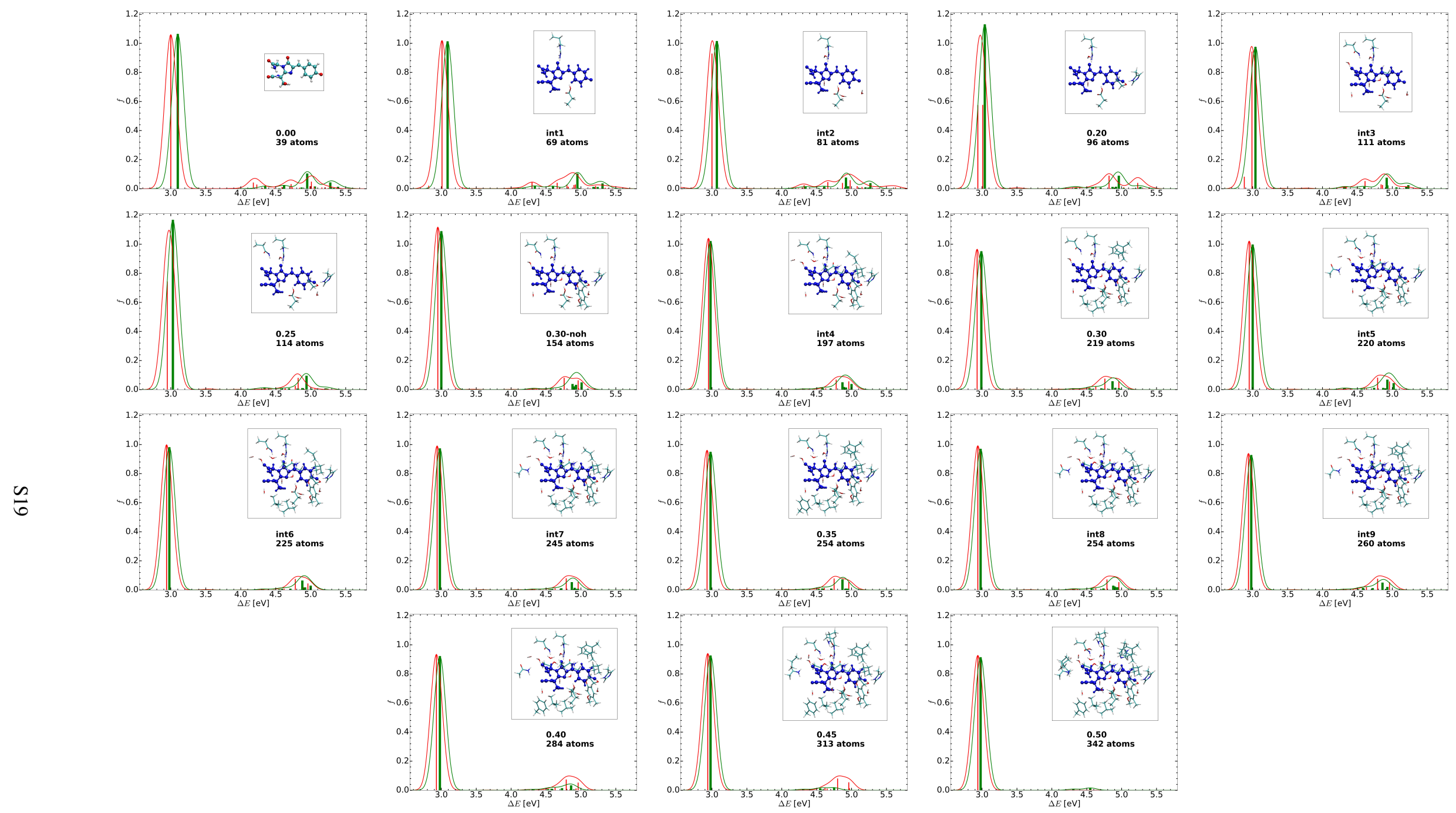

Figure S7: OPA spectra for avGFP-a models. No embedding in red, electrostatic embedding in green. 

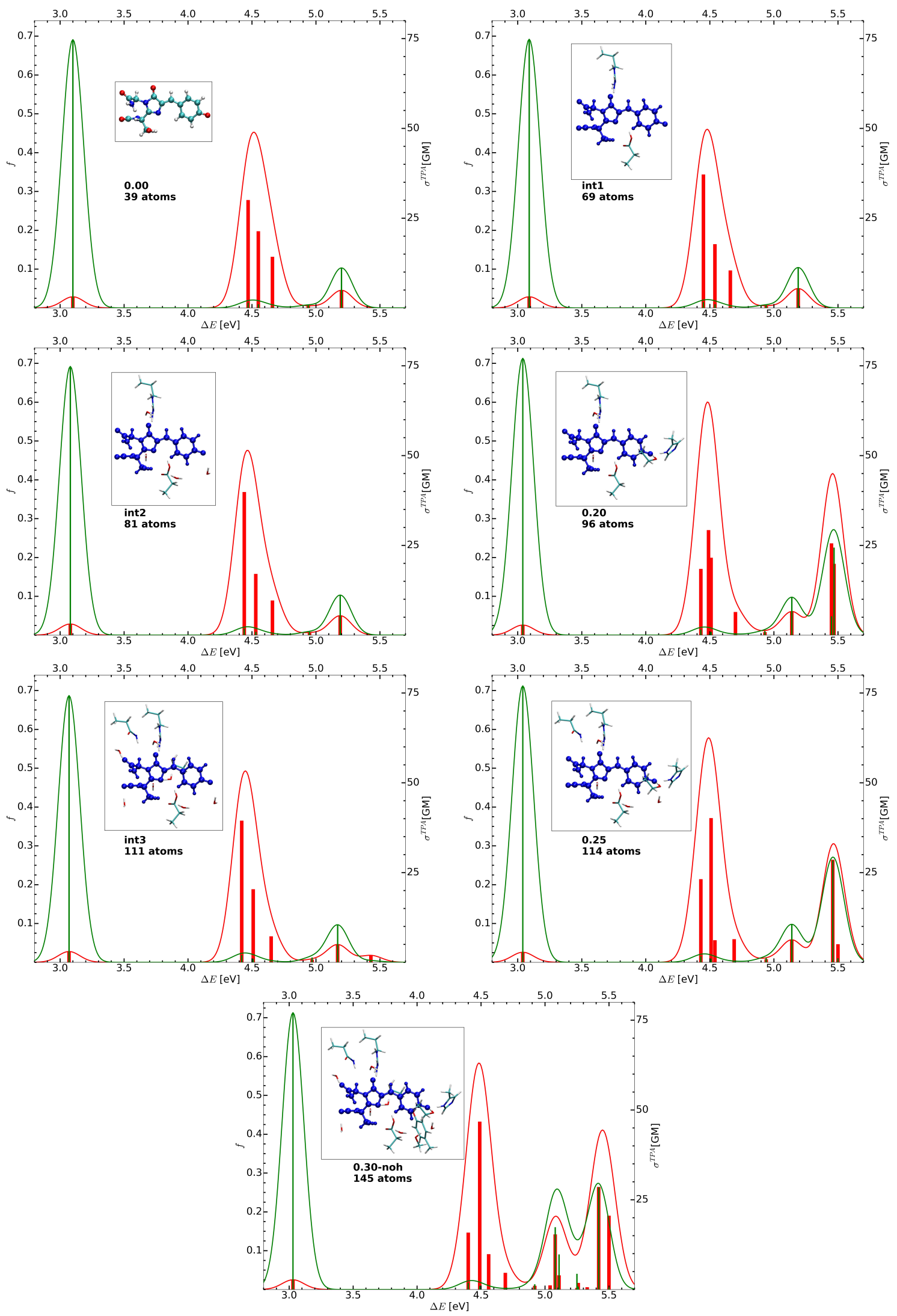

Figure S8: OPA (green) and TPA (red) spectra of avGFP-a models with the polarizable embedding applied. 

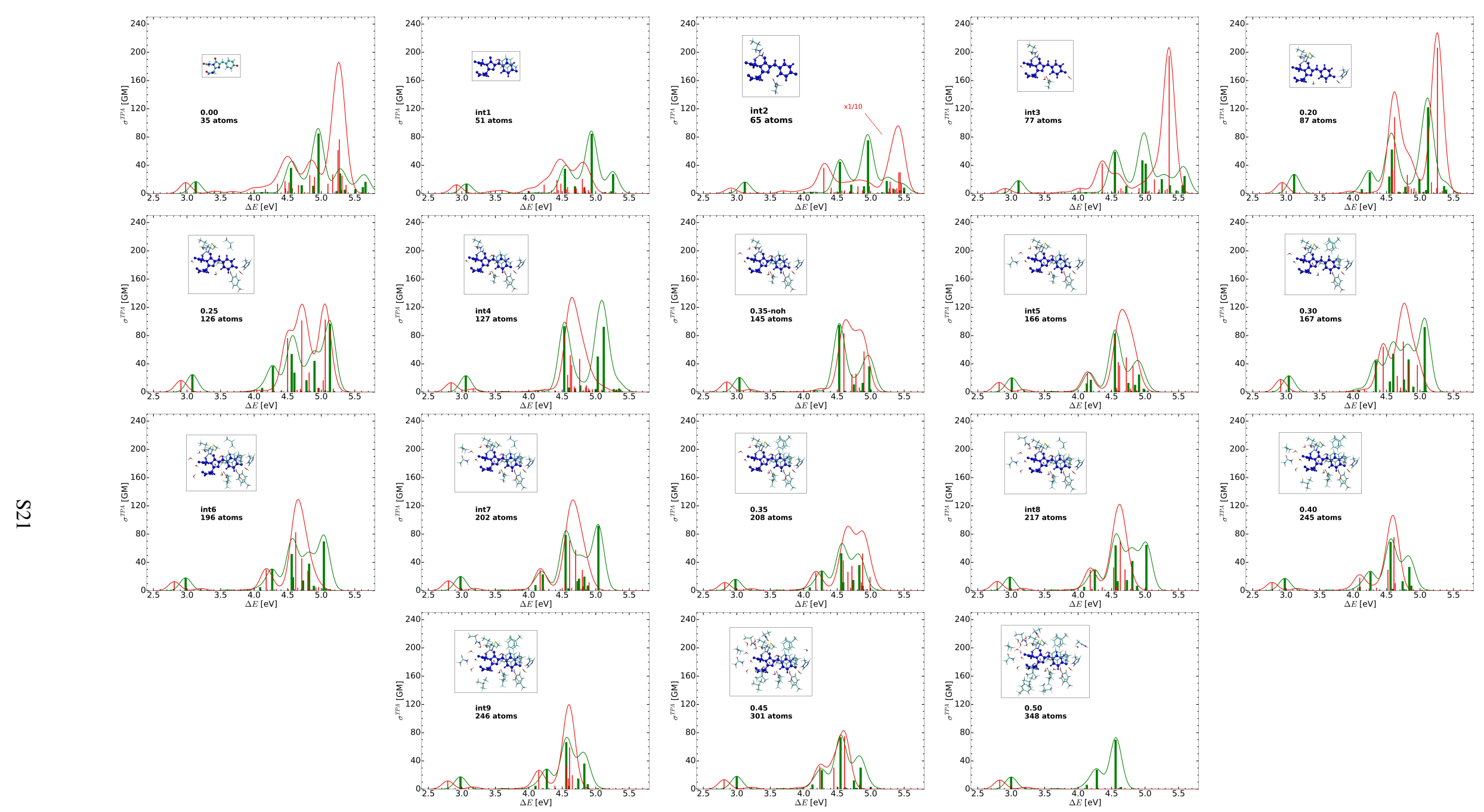

Figure S9: TPA spectra for Citrine models. No embedding in red, electrostatic embedding in green. In case of the int $2 / \mathrm{NEmodels,} \mathrm{we} \mathrm{rescaled} \mathrm{all} \sigma^{T P A}$ values by $1 / 10$ if correspoding $\Delta E>5.0 \mathrm{eV}$. 

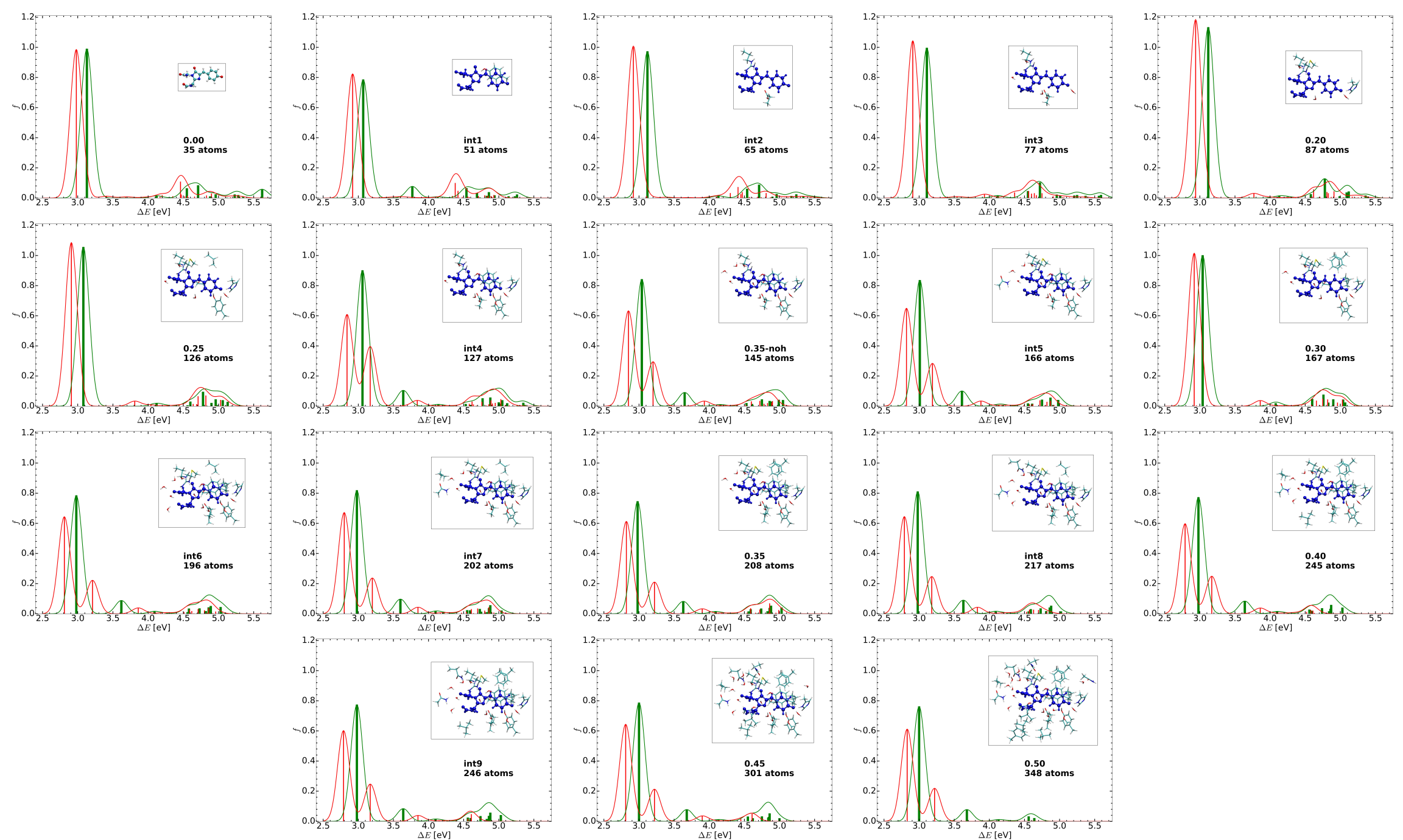

Figure S10: OPA spectra for Citrine models. No embedding in red, electrostatic embedding in green. 

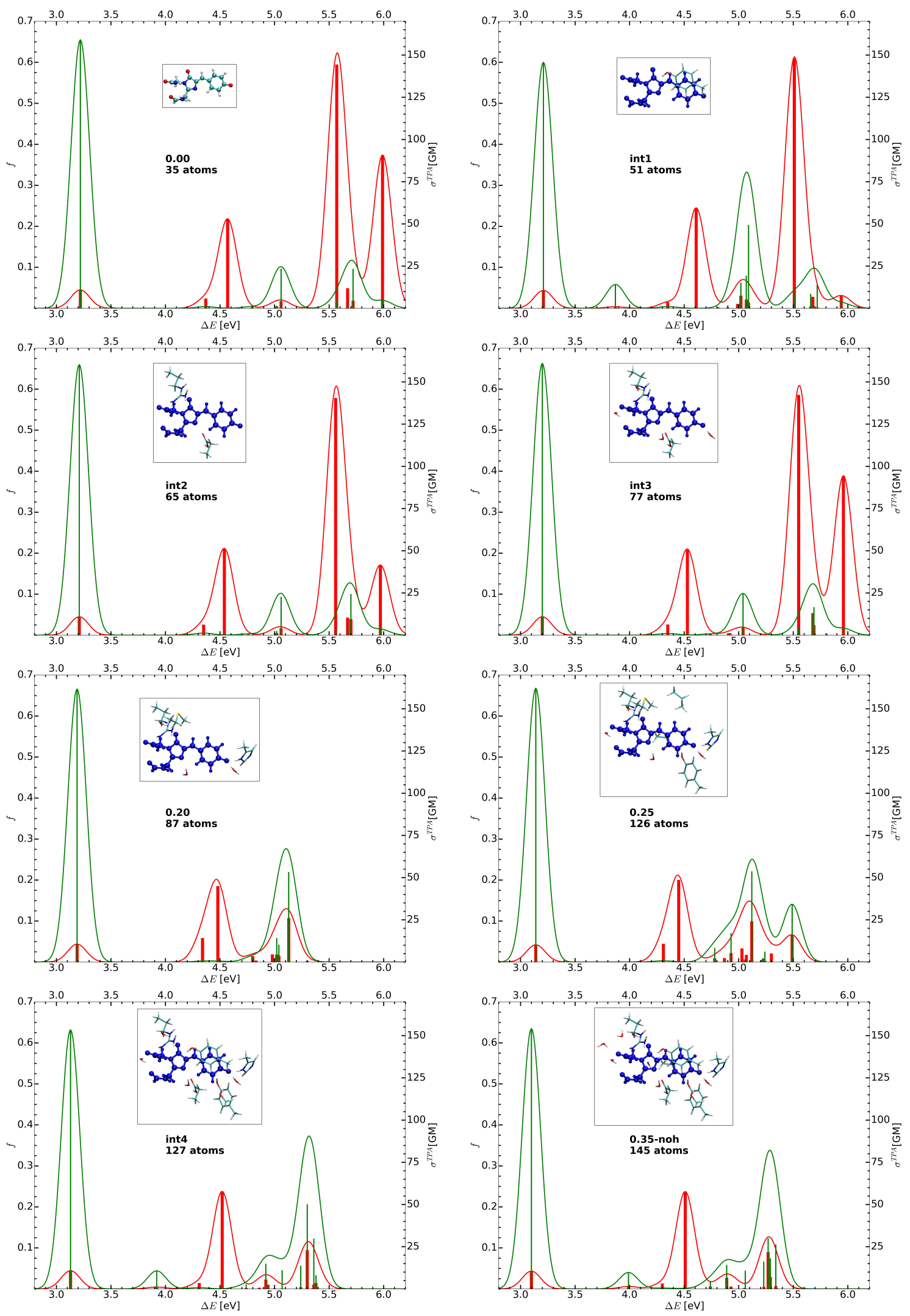

Figure S11: OPA (green) and TPA (red) spectra of Citrine models with the polarizable embedding applied. 


\section{S5 $\triangle E, \sigma^{T P A}$ and $f$ values obtained for different models of FPs}

Table S10: Impact of QM cluster size on excitation energy $(\Delta E[\mathrm{eV}])$, two-photon absorption cross-section $\left(\sigma^{T P A}[\mathrm{GM}]\right)$ and one-photon oscillator strength $(f)$ for avGFP-n. For composition of clusters, see Tables S1-S3. Number of atoms is given in parentheses.

\begin{tabular}{|c|c|c|c|c|c|c|c|c|c|c|c|c|c|c|c|c|c|c|c|c|c|}
\hline \multirow[b]{2}{*}{ State } & \multicolumn{3}{|c|}{$0.00(40)$} & \multicolumn{3}{|c|}{$0.20(56)$} & \multicolumn{3}{|c|}{ int1 (69) } & \multicolumn{3}{|c|}{ int2 (84) } & \multicolumn{3}{|c|}{ int3 (117) } & \multicolumn{3}{|c|}{$0.25(140)$} & \multicolumn{3}{|c|}{ 0.30-noh (141) } \\
\hline & $\Delta E$ & $\sigma^{T P A}$ & $f$ & $\Delta E$ & $\sigma^{T P A}$ & $f$ & $\Delta E$ & $\sigma^{T P A}$ & $f$ & $\Delta E$ & $\sigma^{T P}$ & $f$ & $\Delta E$ & $\sigma^{T P A}$ & $f$ & $\Delta E$ & $\sigma^{T P A}$ & $f$ & $\Delta E$ & $\sigma^{T P A}$ & $f$ \\
\hline$S_{1}$ & 3.31 & 2.5 & 0.817 & 3.28 & 3.9 & 0.814 & 2.95 & 0.1 & 0.000 & 3.15 & 6.8 & 0.798 & 3.13 & 9.3 & 0.924 & 3.13 & 7.6 & 0.843 & 3.12 & 9.0 & 0.877 \\
\hline$S_{2}$ & 4.15 & 4.1 & 0.012 & 4.13 & 0.9 & 0.000 & 3.12 & 2.3 & 0.124 & 3.65 & 2.1 & 0.003 & 3.64 & 0.1 & 0.000 & 3.68 & 0.1 & 0.000 & 3.81 & 6.1 & 0.000 \\
\hline$S_{3}$ & 4.52 & 22.8 & 0.059 & 4.19 & 7.8 & 0.015 & 3.19 & 3.6 & 0.637 & 3.68 & 0.3 & 0.005 & 3.80 & 1.9 & 0.003 & 3.99 & 0.4 & 0.002 & 4.27 & 1.6 & 0.002 \\
\hline$S_{4}$ & 4.57 & 76.7 & 0.018 & 4.47 & 2.5 & 0.008 & 3.33 & 0.1 & 0.001 & 3.75 & 0.1 & 0.000 & 3.88 & 0.4 & 0.004 & 4.03 & 1.9 & 0.003 & 4.31 & 12.2 & 0.007 \\
\hline$S_{5}$ & 4.73 & 0.2 & 0.002 & 4.48 & 0.1 & 0.000 & 3.58 & 0.0 & 0.000 & 4.07 & 0.4 & 0.001 & 3.96 & 0.4 & 0.002 & 4.12 & 0.4 & 0.002 & 4.34 & 43.9 & 0.010 \\
\hline$S_{6}$ & 5.02 & 11.6 & 0.002 & 4.53 & 9.9 & 0.030 & 3.66 & 0.0 & 0.000 & 4.18 & 0.4 & 0.002 & 4.16 & 1.9 & 0.002 & 4.17 & 10.9 & 0.001 & 4.40 & 2.6 & 0.001 \\
\hline$S_{7}$ & 5.17 & 97.8 & 0.020 & 4.56 & 1.3 & 0.003 & 3.70 & 2.4 & 0.004 & 4.26 & 8.8 & 0.006 & 4.23 & 11.3 & 0.001 & 4.20 & 2.4 & 0.002 & 4.42 & 1.4 & 0.001 \\
\hline$S_{8}$ & 5.29 & 15.7 & 0.007 & 4.59 & 91.9 & 0.020 & 3.74 & 0.2 & 0.004 & 4.32 & 0.0 & 0.000 & 4.32 & 48.0 & 0.020 & 4.30 & 45.5 & 0.018 & 4.50 & 3.5 & 0.001 \\
\hline$S_{9}$ & 5.31 & 35.6 & 0.008 & 4.64 & 0.1 & 0.007 & 3.82 & 0.0 & 0.000 & 4.33 & 27.8 & 0.013 & 4.36 & 3.1 & 0.002 & 4.49 & 6.3 & 0.000 & 4.62 & 50.1 & 0.007 \\
\hline$S_{10}$ & 5.36 & 7.6 & 0.004 & 4.66 & 0.6 & 0.006 & 3.88 & 0.1 & 0.000 & 4.41 & 0.0 & 0.000 & 4.43 & 0.0 & 0.000 & 4.60 & 43.8 & 0.010 & 4.68 & 0.0 & 0.000 \\
\hline$S_{11}$ & & & & 4.81 & 7.1 & 0.002 & 4.02 & 0.0 & 0.000 & 4.50 & 7.6 & 0.001 & 4.46 & 0.0 & 0.000 & 4.65 & 2.6 & 0.002 & 4.72 & 0.0 & 0.000 \\
\hline$S_{12}$ & & & & 4.84 & 0.1 & 0.000 & 4.07 & 0.0 & 0.000 & 4.60 & 24.0 & 0.007 & 4.49 & 2.1 & 0.001 & 4.68 & 0.0 & 0.000 & 4.83 & 0.0 & 0.000 \\
\hline$S_{13}$ & & & & 5.00 & 73.5 & 0.017 & 4.09 & 0.0 & 0.000 & 4.62 & 18.3 & 0.005 & 4.63 & 52.9 & 0.012 & 4.73 & 0.0 & 0.000 & 4.88 & 2.5 & 0.004 \\
\hline$S_{14}$ & & & & 5.09 & 23.5 & 0.005 & 4.18 & 23.5 & 0.033 & 4.71 & 0.0 & 0.000 & 4.64 & 0.0 & 0.000 & 4.77 & 0.0 & 0.000 & 5.02 & 1.2 & 0.018 \\
\hline$S_{15}$ & & & & 5.09 & 3.7 & 0.001 & 4.24 & 0.5 & 0.001 & 4.77 & 0.5 & 0.000 & 4.72 & 0.0 & 0.000 & 4.87 & 0.7 & 0.007 & 5.13 & 12.3 & 0.096 \\
\hline$S_{16}$ & & & & & & & 4.30 & 0.0 & 0.000 & 4.80 & 0.0 & 0.000 & 4.82 & 0.0 & 0.000 & 4.89 & 0.0 & 0.000 & & & \\
\hline$S_{17}$ & & & & & & & 4.46 & 0.0 & 0.000 & 4.81 & 0.0 & 0.000 & 4.84 & 0.0 & 0.000 & 5.04 & 0.5 & 0.004 & & & \\
\hline$S_{18}$ & & & & & & & 4.48 & 33.9 & 0.015 & 4.91 & 0.3 & 0.000 & 4.87 & 0.2 & 0.002 & 5.05 & 0.0 & 0.000 & & & \\
\hline$S_{19}$ & & & & & & & 4.50 & 0.0 & 0.000 & 4.91 & 1.2 & 0.001 & 4.88 & 0.7 & 0.007 & 5.06 & 4.0 & 0.002 & & & \\
\hline$S_{20}$ & & & & & & & 4.58 & 22.2 & 0.009 & 5.00 & 0.0 & 0.000 & 4.89 & 0.0 & 0.000 & 5.09 & 0.1 & 0.000 & & & \\
\hline$S_{21}$ & & & & & & & 4.66 & 0.0 & 0.000 & 5.13 & 0.6 & 0.064 & 4.95 & 0.2 & 0.000 & 5.09 & 0.6 & 0.001 & & & \\
\hline$S_{22}$ & & & & & & & 4.68 & 0.1 & 0.001 & 5.14 & 0.6 & 0.017 & 4.96 & 0.6 & 0.006 & 5.13 & 11.5 & 0.131 & & & \\
\hline$S_{23}$ & & & & & & & 4.71 & 0.1 & 0.001 & 5.16 & 6.5 & 0.050 & 5.03 & 0.0 & 0.000 & 5.23 & 0.0 & 0.000 & & & \\
\hline$S_{24}$ & & & & & & & 4.83 & 0.2 & 0.000 & 5.17 & 0.0 & 0.000 & 5.05 & 0.0 & 0.000 & 5.23 & 5.7 & 0.001 & & & \\
\hline$S_{25}$ & & & & & & & 4.85 & 0.0 & 0.000 & 5.20 & 9.0 & 0.002 & 5.07 & 2.4 & 0.007 & 5.27 & 12.3 & 0.005 & & & \\
\hline- & & & & & & & Cont & ued on $\mathrm{F}$ & ge S26 & & & & & & & & & & & & \\
\hline & & int $4(14$ & & & 35-noh (1 & & & int5 (20 & & & int6 (20 & & & int7 (23 & & & $0.30(23$ & & & int8 (242 & \\
\hline State & $\Delta E$ & $\sigma^{T P A}$ & $f$ & $\Delta E$ & $\sigma^{T P A}$ & $f$ & $\Delta E$ & $\sigma^{T P A}$ & $f$ & $\Delta E$ & $\sigma^{T P A}$ & $f$ & $\Delta E$ & $\sigma^{T P A}$ & $f$ & $\Delta E$ & $\sigma^{T P A}$ & $f$ & $\Delta E$ & $\sigma^{T P A}$ & $f$ \\
\hline$S_{1}$ & 3.13 & 9.5 & 0.899 & 3.13 & 8.8 & 0.853 & 3.11 & 9.2 & 0.886 & 3.12 & 7.9 & $\begin{array}{l}0.814 \\
\end{array}$ & 3.12 & 8.4 & 0.830 & 3.10 & 7.7 & 0.841 & 3.12 & 8.9 & 0.856 \\
\hline$S_{2}$ & 4.05 & 7.4 & 0.000 & 3.78 & 5.6 & 0.000 & 3.85 & 1. & 0.000 & 3.86 & 1.6 & 0.000 & 3.81 & 1. & 0.000 & 3.94 & 1.7 & 0.000 & 3.80 & 1.7 & 0.000 \\
\hline$S_{3}$ & 4.11 & 0.2 & 0.003 & 4.13 & 0.1 & 0.000 & 4.31 & 28.8 & 0.009 & 4.20 & 0.1 & 0.000 & 4.15 & 0.1 & 0.000 & 4.33 & 57.0 & 0.010 & 4.14 & 0.1 & 0.000 \\
\hline$S_{4}$ & 4.19 & 2.3 & 0.002 & 4.33 & 40.6 & 0.014 & 4.33 & 26.9 & 0.007 & 4.31 & 50.5 & 0.017 & 4.33 & 53.0 & 0.013 & 4.40 & 1.7 & 0.005 & 4.33 & 55.7 & 0.014 \\
\hline$S_{5}$ & 4.34 & 52.4 & 0.020 & 4.36 & 12.0 & 0.002 & 4.36 & 9.2 & 0.001 & 4.45 & 4.9 & 0.001 & 4.45 & 4.8 & 0.001 & 4.40 & 0.7 & 0.000 & 4.43 & 4.3 & 0.001 \\
\hline$S_{6}$ & 4.45 & 3.4 & 0.001 & 4.50 & 6.7 & 0.001 & 4.41 & 3.3 & 0.001 & 4.51 & 8.4 & 0.001 & 4.52 & 6.9 & 0.000 & 4.47 & 0.9 & 0.002 & 4.51 & 8.3 & 0.000 \\
\hline$S_{7}$ & 4.53 & 3.8 & 0.000 & 4.52 & 4.1 & 0.000 & 4.48 & 1.9 & 0.001 & 4.56 & 12.1 & 0.000 & 4.61 & 40.3 & 0.003 & 4.55 & 0.2 & 0.000 & 4.59 & 5.0 & 0.001 \\
\hline$S_{8}$ & 4.58 & 0.5 & 0.000 & 4.61 & 45.6 & 0.002 & 4.51 & 1.3 & 0.000 & 4.60 & 18.2 & 0.003 & 4.62 & 18.0 & 0.001 & 4.59 & 0.8 & 0.002 & 4.62 & 57.4 & 0.004 \\
\hline$S_{9}$ & 4.60 & 4.4 & 0.004 & 4.63 & 12.0 & 0.008 & 4.62 & 57.4 & 0.006 & 4.61 & 24.1 & 0.005 & 4.64 & 2.3 & 0.005 & 4.62 & 58.4 & 0.006 & 4.63 & 2.6 & 0.005 \\
\hline$S_{10}$ & 4.64 & 50.5 & 0.010 & 4.75 & 2.3 & 0.002 & 4.73 & 0.0 & 0.000 & 4.67 & 1.8 & 0.001 & 4.76 & 2.2 & 0.002 & 4.86 & 0.0 & 0.000 & 4.75 & 2.2 & 0.002 \\
\hline$S_{11}$ & 4.71 & 0.0 & & 4.82 & 0.0 & & 4.80 & 0.0 & & & 0.0 & & 4.93 & 0.0 & 0.000 & 4.93 & 1.5 & 0.007 & 4.90 & 0.0 & 0.000 \\
\hline$S_{12}$ & 4.88 & 0.0 & 0.000 & 4.89 & 0.1 & 0.000 & 4.88 & 0.0 & 0.000 & 4.91 & 0.0 & 0.000 & 4.94 & 0.0 & 0.000 & 5.01 & 0.0 & 0.000 & 4.93 & 0.0 & 0.000 \\
\hline$S_{13}$ & 4.89 & 0.0 & 0.000 & 4.99 & 0.0 & 0.057 & 4.89 & 3.1 & 0.004 & 4.99 & 0.0 & 0.050 & 4.99 & 0.0 & 0.051 & 5.04 & 6.4 & 0.115 & 4.99 & 0.0 & 0.051 \\
\hline
\end{tabular}




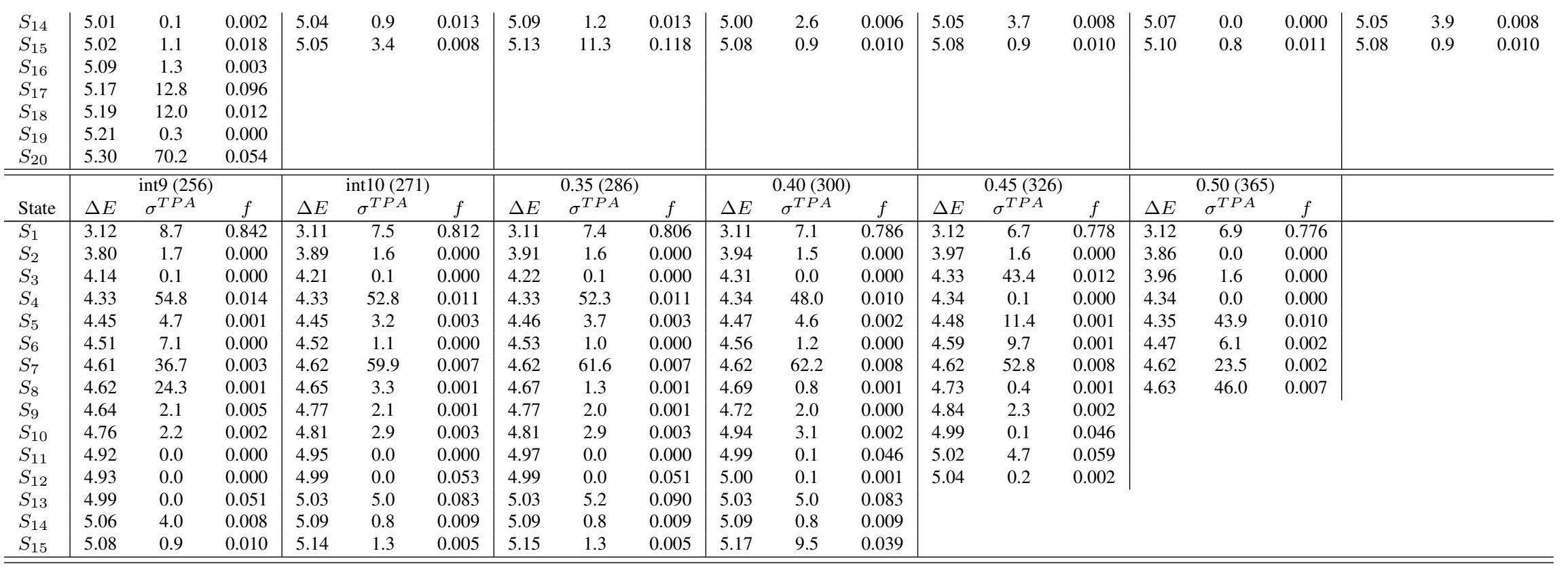


Table S10 continued for the int1 QM cluster.

\begin{tabular}{l|ccc} 
& \multicolumn{3}{|c}{ int1 (69) } \\
State & $\Delta E$ & $\sigma^{T P A}$ & $f$ \\
\hline$S_{26}$ & 4.90 & 0.1 & 0.000 \\
$S_{27}$ & 4.90 & 0.0 & 0.000 \\
$S_{28}$ & 4.92 & 0.1 & 0.002 \\
$S_{29}$ & 4.94 & 0.0 & 0.001 \\
$S_{30}$ & 4.96 & 2.3 & 0.001 \\
$S_{31}$ & 4.99 & 0.8 & 0.000 \\
$S_{32}$ & 5.07 & 0.2 & 0.000 \\
$S_{33}$ & 5.08 & 0.1 & 0.000 \\
$S_{34}$ & 5.10 & 0.0 & 0.000 \\
$S_{35}$ & 5.13 & 0.0 & 0.000 \\
$S_{36}$ & 5.18 & 2.7 & 0.125 \\
$S_{37}$ & 5.22 & 0.1 & 0.000 \\
$S_{38}$ & 5.22 & 2.0 & 0.008 \\
$S_{39}$ & 5.23 & 13.1 & 0.005 \\
$S_{40}$ & 5.26 & 6.1 & 0.002 \\
$S_{41}$ & 5.28 & 0.2 & 0.000 \\
$S_{42}$ & 5.28 & 2.4 & 0.002 \\
$S_{43}$ & 5.33 & 1.0 & 0.003 \\
$S_{44}$ & 5.35 & 0.2 & 0.001 \\
$S_{45}$ & 5.36 & 0.5 & 0.001 \\
$S_{46}$ & 5.36 & 3.2 & 0.003 \\
$S_{47}$ & 5.36 & 12.2 & 0.001 \\
$S_{48}$ & 5.38 & 8.5 & 0.019 \\
$S_{49}$ & 5.39 & 0.8 & 0.001 \\
$S_{50}$ & 5.40 & 0.9 & 0.001 \\
\hline \hline
\end{tabular}


Table S11: Impact of QM cluster size on excitation energy ( $\Delta E[\mathrm{eV}])$, two-photon absorption cross-section $\left(\sigma^{T P A}\right.$ [GM]) and one-photon oscillator strength $(f)$ for avGFP-n. For composition of clusters, see Tables S1-S3. Number of atoms is given in parentheses. The MM subsystem is described by electrostatic embedding.

\begin{tabular}{|c|c|c|c|c|c|c|c|c|c|c|c|c|c|c|c|c|c|c|c|c|c|}
\hline \multirow[b]{2}{*}{ State } & \multicolumn{3}{|c|}{$0.00(40)$} & \multicolumn{3}{|c|}{$0.20(56)$} & \multicolumn{3}{|c|}{ int1 (69) } & \multicolumn{3}{|c|}{ int2 (84) } & \multicolumn{3}{|c|}{ int3 (117) } & \multicolumn{3}{|c|}{$0.25(140)$} & \multicolumn{3}{|c|}{ 0.30-noh (141) } \\
\hline & $\Delta E$ & $\sigma^{T P A}$ & $f$ & $\Delta E$ & $\sigma^{T P A}$ & $f$ & $\Delta E$ & $\sigma^{T P A}$ & $f$ & $\Delta E$ & $\sigma^{T P A}$ & $f$ & $\Delta E$ & $\sigma^{T P A}$ & $f$ & $\Delta E$ & & $f$ & $\Delta E$ & $\sigma^{T P A}$ & $f$ \\
\hline$S_{1}$ & 3.28 & 4.8 & 0.858 & 3.25 & 4.9 & 0.821 & 3.25 & 4.3 & 0.767 & 3.23 & 5.3 & 0.782 & 3.22 & 7.0 & 0.892 & 3.20 & 5.8 & 0.826 & 3.19 & 6.9 & 0.853 \\
\hline$S_{2}$ & 4.31 & 1.6 & 0.001 & 4.28 & 1.4 & 0.001 & 4.26 & 1.5 & 0.001 & 4.27 & 1.5 & 0.001 & 4.33 & 34.1 & 0.013 & 4.31 & 0.6 & 0.001 & 4.25 & 1.3 & 0.001 \\
\hline$S_{3}$ & 4.35 & 24.8 & 0.022 & 4.34 & 24.9 & 0.021 & 4.33 & 22.2 & 0.022 & 4.35 & 27.3 & 0.020 & 4.35 & 9.4 & 0.012 & 4.33 & 34.9 & 0.020 & 4.29 & 12.9 & 0.002 \\
\hline$S_{4}$ & 4.57 & 22.0 & 0.026 & 4.56 & 19.8 & 0.026 & 4.56 & 24.8 & 0.022 & 4.56 & 22.8 & 0.019 & 4.39 & 2.0 & 0.001 & 4.41 & 5.9 & 0.000 & 4.35 & 30.9 & 0.016 \\
\hline$S_{5}$ & 4.65 & 73.1 & 0.017 & 4.64 & 70.9 & 0.017 & 4.64 & 58.2 & 0.019 & 4.64 & 61.3 & 0.018 & 4.56 & 39.3 & 0.023 & 4.54 & 33.9 & 0.015 & 4.54 & 32.2 & 0.010 \\
\hline$S_{6}$ & 4.89 & 6.0 & 0.001 & 4.81 & 5.8 & 0.001 & 4.70 & 0.4 & 0.005 & 4.87 & 3.1 & 0.003 & 4.64 & 70.4 & 0.015 & 4.62 & 65.9 & 0.015 & 4.63 & 72.2 & 0.013 \\
\hline$S_{7}$ & 5.29 & 39.4 & 0.006 & 4.84 & 0.6 & 0.004 & 4.75 & 0.2 & 0.001 & 4.90 & 0.4 & 0.002 & 4.86 & 2.3 & 0.036 & 4.85 & 2.5 & 0.035 & 4.86 & 2.7 & 0.038 \\
\hline$S_{8}$ & 5.31 & 35.3 & 0.052 & 4.90 & 0.2 & 0.001 & 4.89 & 2.7 & 0.002 & 4.99 & 0.4 & 0.002 & 4.89 & 2.0 & 0.005 & 4.94 & 0.3 & 0.003 & 5.03 & 0.3 & 0.004 \\
\hline$S_{9}$ & 5.38 & 13.4 & 0.089 & 5.19 & 1.1 & 0.000 & 5.04 & 0.3 & 0.000 & 5.21 & 26.3 & 0.007 & 4.90 & 2.9 & 0.001 & 5.03 & 0.4 & 0.002 & 5.04 & 0.6 & 0.003 \\
\hline$S_{10}$ & 5.47 & 22.1 & 0.013 & 5.25 & 41.8 & 0.024 & 5.23 & 32.3 & 0.010 & 5.26 & 0.9 & 0.000 & 4.99 & 0.4 & 0.002 & 5.04 & 0.6 & 0.003 & 5.06 & 0.0 & 0.000 \\
\hline$S_{11}$ & & & & 5.29 & 5.4 & 0.003 & 5.28 & 23.0 & 0.004 & 5.29 & 33.2 & 0.006 & 5.23 & 36.1 & 0.008 & 5.21 & 1.1 & 0.002 & 5.12 & 0.2 & 0.004 \\
\hline$S_{12}$ & & & & 5.32 & 27.9 & 0.002 & 5.35 & 3.1 & 0.121 & 5.32 & 3.3 & 0.051 & 5.23 & 11.7 & 0.003 & 5.23 & 1.7 & 0.000 & 5.22 & 103.4 & 0.036 \\
\hline$S_{13}$ & & & & 5.37 & 2.6 & 0.121 & 5.47 & 48.8 & 0.029 & 5.34 & 2.1 & 0.076 & 5.25 & 0.9 & 0.001 & 5.23 & 108.7 & 0.022 & 5.25 & 7.6 & 0.071 \\
\hline$S_{14}$ & & & & 5.46 & 54.9 & 0.036 & 5.49 & 3.6 & 0.007 & 5.45 & 55.4 & 0.031 & 5.28 & 4.2 & 0.001 & 5.28 & 3.9 & 0.080 & 5.29 & 3.1 & 0.013 \\
\hline$S_{15}$ & & & & 5.59 & 3.1 & 0.015 & 5.50 & 0.5 & 0.000 & 5.52 & 3.5 & 0.007 & 5.33 & 27.8 & 0.040 & 5.30 & 2.6 & 0.021 & 5.34 & 11.2 & 0.003 \\
\hline & \multicolumn{3}{|c|}{ int4 (144) } & \multicolumn{3}{|c|}{0.35 -noh (178) } & \multicolumn{3}{|c|}{ int5 (205) } & \multicolumn{3}{|c|}{ int6 (208) } & \multicolumn{3}{|c|}{ int7 (231) } & \multicolumn{3}{|c|}{$0.30(234$} & \multicolumn{3}{|c|}{ int8 (242) } \\
\hline State & $\Delta E$ & $\sigma^{T P A}$ & $f$ & $\Delta E$ & $\sigma^{T P A}$ & $f$ & $\Delta E$ & $\sigma^{T P}$ & $f$ & $\Delta E$ & $\sigma^{T H}$ & $f$ & $\Delta E$ & $\sigma^{T H}$ & $f$ & $\Delta E$ & $\sigma^{T}$ & $f$ & $\Delta E$ & $\sigma^{T P A}$ & $f$ \\
\hline$S_{1}$ & 3.20 & 7.7 & 0.869 & 3.17 & 7.2 & 0.835 & 3.18 & 7.1 & 0.865 & 3.16 & 7.2 & 0.809 & 3.16 & 7.3 & 0.818 & 3.17 & 5.9 & 0.829 & 3.16 & 7.3 & 0.840 \\
\hline$S_{2}$ & 4.16 & 1.0 & 0.001 & 4.06 & 1.0 & 0.001 & 4.22 & 1.4 & 0.001 & 3.95 & 1.0 & 0.001 & 3.99 & 1.0 & 0.001 & 4.22 & 1.5 & 0.001 & 4.04 & 1.0 & 0.001 \\
\hline$S_{3}$ & 4.27 & 10.8 & 0.001 & 4.30 & 12.7 & 0.002 & 4.27 & 2.9 & 0.001 & 4.20 & 2.0 & 0.000 & 4.23 & 2.1 & 0.000 & 4.35 & 40.0 & 0.014 & 4.28 & 2.6 & 0.001 \\
\hline$S_{4}$ & 4.36 & 34.0 & 0.022 & 4.36 & 30.4 & 0.015 & 4.34 & 40.8 & 0.016 & 4.37 & 39.0 & 0.014 & 4.36 & 39.8 & 0.014 & 4.36 & 0.5 & 0.001 & 4.35 & 40.1 & 0.016 \\
\hline$S_{5}$ & 4.56 & 32.8 & 0.017 & 4.50 & 0.1 & 0.000 & 4.53 & 35.2 & 0.010 & 4.47 & 0.0 & 0.000 & 4.50 & 0.1 & 0.000 & 4.51 & 20.1 & 0.007 & 4.53 & 30.5 & 0.007 \\
\hline$S_{6}$ & 4.64 & 73.7 & 0.016 & 4.54 & 31.2 & 0.007 & 4.63 & 80.2 & 0.012 & 4.54 & 26.6 & 0.005 & 4.54 & 28.3 & 0.006 & 4.62 & 87.5 & 0.016 & 4.54 & 3.5 & 0.001 \\
\hline$S_{7}$ & 4.84 & 3.8 & 0.001 & 4.63 & 75.0 & 0.014 & 4.78 & 2.9 & 0.030 & 4.64 & 0.0 & 0.000 & 4.64 & 79.7 & 0.013 & 4.79 & 2.8 & 0.029 & 4.63 & 81.8 & 0.013 \\
\hline$S_{8}$ & 4.88 & 2.8 & 0.041 & 4.85 & 0.0 & 0.000 & 5.00 & 0.0 & 0.000 & 4.64 & 77.3 & 0.013 & 4.71 & 0.0 & 0.000 & 5.03 & 0.3 & 0.027 & 4.78 & 2.9 & 0.029 \\
\hline$S_{9}$ & 4.92 & 0.0 & 0.000 & 4.87 & 2.7 & 0.041 & 5.00 & 1.0 & 0.004 & 4.78 & 2.9 & 0.029 & 4.78 & 2.9 & 0.029 & 5.07 & 8.8 & 0.055 & 4.80 & 0.0 & 0.000 \\
\hline$S_{10}$ & 5.01 & 0.3 & 0.003 & 5.00 & 0.4 & 0.004 & 5.10 & 0.4 & 0.010 & 4.90 & 0.0 & 0.000 & 4.97 & 0.0 & 0.000 & 5.07 & 0.0 & 0.000 & 5.01 & 0.1 & 0.048 \\
\hline$S_{11}$ & 5.10 & 0.3 & 0.002 & 5.01 & 0.2 & 0.045 & 5.18 & 0.4 & 0.011 & 5.01 & 0.2 & 0.048 & 5.01 & 0.2 & 0.048 & 5.09 & 2.6 & 0.003 & 5.01 & 1.1 & 0.002 \\
\hline$S_{12}$ & 5.15 & 30.6 & 0.005 & 5.03 & 0.0 & 0.000 & 5.21 & 74.0 & 0.018 & 5.03 & 2.1 & 0.003 & 5.02 & 2.1 & 0.003 & 5.17 & 0.7 & 0.002 & 5.06 & 0.0 & 0.000 \\
\hline$S_{13}$ & 5.27 & 30.0 & 0.003 & 5.04 & 0.3 & 0.004 & 5.24 & 4.6 & 0.091 & 5.08 & 0.3 & 0.007 & 5.05 & 0.3 & 0.005 & 5.21 & 43.1 & 0.009 & 5.10 & 0.3 & 0.008 \\
\hline$S_{14}$ & 5.31 & 10.1 & 0.104 & 5.13 & 0.2 & 0.004 & 5.28 & 12.2 & 0.010 & 5.13 & 37.8 & 0.006 & 5.14 & 19.8 & 0.003 & 5.22 & 20.2 & 0.001 & 5.16 & 5.4 & 0.039 \\
\hline$S_{15}$ & 5.37 & 3.5 & 0.001 & 5.18 & 77.4 & 0.014 & 5.29 & 14.7 & 0.015 & 5.16 & 2.5 & 0.036 & 5.15 & 28.7 & 0.011 & 5.25 & 4.0 & 0.036 & 5.18 & 59.1 & 0.005 \\
\hline & & int9 $(25$ & & & int10 (2) & & & $0.35(28$ & & & $0.40(30$ & & & $0.45(32$ & & & $0.50(36$ & & & & \\
\hline State & $\Delta E$ & $\sigma^{T P A}$ & $f$ & $\Delta E$ & $\sigma^{T P}$ & $f$ & $\Delta E$ & $\sigma^{T 1}$ & $f$ & $\Delta E$ & $\sigma^{T P A}$ & $f$ & $\Delta E$ & $\sigma^{T H}$ & $f$ & $\Delta E$ & $\sigma^{T}$ & $f$ & & & \\
\hline$S_{1}$ & 3.16 & 7.0 & 0.825 & 3.16 & 6.1 & 0.802 & 3.16 & 5.9 & 0.797 & 3.16 & 5.8 & 0.779 & 3.16 & 5.4 & 0.770 & 3.16 & 5.5 & 0.763 & & & \\
\hline$S_{2}$ & 4.07 & 1.0 & 0.001 & 4.04 & 1.0 & 0.001 & 4.04 & 1.0 & 0.001 & 3.97 & 1.0 & 0.001 & 3.96 & 0.9 & 0.001 & 3.90 & 0.9 & 0.000 & & & \\
\hline$S_{3}$ & 4.30 & 3.3 & 0.001 & 4.35 & 33.9 & 0.012 & 4.35 & 38.9 & 0.015 & 4.35 & 36.2 & 0.014 & 4.34 & 32.9 & 0.014 & 4.35 & 31.8 & 0.014 & & & \\
\hline$S_{4}$ & 4.35 & 38.1 & 0.016 & 4.36 & 5.7 & 0.003 & 4.37 & 0.2 & 0.000 & 4.39 & 0.7 & 0.000 & 4.46 & 1.1 & 0.000 & 4.51 & 1.6 & 0.002 & & & \\
\hline$S_{5}$ & 4.53 & 32.5 & 0.008 & 4.52 & 19.5 & 0.005 & 4.52 & 19.4 & 0.006 & 4.51 & 18.5 & 0.005 & 4.51 & 20.2 & 0.008 & 4.52 & 19.9 & 0.006 & & & \\
\hline$S_{6}$ & 4.57 & 0.9 & 0.000 & 4.61 & 1.6 & 0.000 & 4.62 & 4.5 & 0.001 & 4.56 & 0.3 & 0.000 & 4.62 & 85.4 & 0.018 & 4.63 & 86.1 & 0.018 & & & \\
\hline$S_{7}$ & 4.63 & 79.7 & 0.013 & 4.63 & 85.8 & 0.017 & 4.63 & 83.4 & 0.017 & 4.63 & 85.6 & 0.018 & 4.63 & 1.1 & 0.000 & 4.70 & 0.0 & 0.000 & & & \\
\hline$S_{8}$ & 4.78 & 2.9 & 0.029 & 4.78 & 2.8 & 0.028 & 4.78 & 2.8 & 0.028 & 4.76 & 2.8 & 0.027 & 4.76 & 2.8 & 0.027 & 4.77 & 2.8 & 0.027 & & & \\
\hline$S_{9}$ & 4.85 & 0.0 & 0.000 & 4.88 & 0.0 & 0.000 & 4.88 & 0.0 & 0.000 & 4.84 & 0.0 & 0.000 & 4.88 & 0.3 & 0.002 & & & & & & \\
\hline$S_{10}$ & 5.00 & 1.0 & 0.003 & 5.01 & 0.3 & 0.048 & 5.01 & 0.3 & 0.047 & 4.99 & 0.2 & 0.007 & 4.89 & 0.0 & 0.000 & & & & & & \\
\hline
\end{tabular}




\begin{tabular}{l|l|l|ll|lll|lll|l}
$S_{11}$ & & 5.03 & 0.2 & 0.018 & 5.00 & 0.3 & 0.042 & 5.00 & 0.4 & 0.041 \\
$S_{12}$ & & 5.08 & 7.7 & 0.049 & 5.00 & 0.0 & 0.000 & 5.05 & 0.0 & 0.000 & \\
$S_{13}$ & & 5.10 & 6.0 & 0.015 & & & & 5.07 & 1.4 & 0.013 & \\
$S_{14}$ & & 5.12 & 0.0 & 0.000 & & & & 5.10 & 6.1 & 0.071 \\
$S_{15}$ & & 5.16 & 6.0 & 0.039 & & & & 5.14 & 4.0 & 0.035 & \\
\hline \hline
\end{tabular}

Table S12: Impact of QM cluster size on excitation energy $(\Delta E[\mathrm{eV}])$, two-photon absorption cross-section $\left(\sigma^{T P A}[\mathrm{GM}]\right)$ and one-photon oscillator strength $(f)$ for avGFP-n. For composition of clusters, see Tables S1-S3. Number of atoms is given in parentheses. The MM subsystem is described by the polarizable embedding.

\begin{tabular}{|c|c|c|c|c|c|c|c|c|c|c|c|c|c|c|c|c|c|c|c|c|c|}
\hline \multirow[b]{2}{*}{ State } & \multicolumn{3}{|c|}{$0.00(40)$} & \multicolumn{3}{|c|}{$0.20(56)$} & \multicolumn{3}{|c|}{ int1 (69) } & \multicolumn{3}{|c|}{ int2 (84) } & \multicolumn{3}{|c|}{ int3 (117) } & \multicolumn{3}{|c|}{$0.25(140)$} & \multicolumn{3}{|c|}{ 0.30-noh (141) } \\
\hline & $\Delta E$ & $\sigma^{T P A}$ & $f$ & $\Delta E$ & $\sigma^{T P A}$ & $f$ & $\Delta E$ & & $f$ & $\Delta E$ & $\sigma^{T P A}$ & $f$ & $\Delta E$ & $\sigma^{T P A}$ & $f$ & $\Delta E$ & $\sigma^{T P A}$ & $f$ & $\Delta E$ & $\sigma^{T P A}$ & $f$ \\
\hline$S_{1}$ & 3.26 & 0.7 & 0.493 & 3.26 & 0.8 & 0.499 & 3.24 & 0.8 & 0.492 & 3.24 & 0.9 & 0.497 & 3.23 & 0.9 & 0.501 & 3.22 & 0.9 & 0.500 & 3.22 & 1.1 & 0.509 \\
\hline$S_{2}$ & 4.25 & 6.9 & 0.021 & 4.26 & 7.4 & 0.020 & 4.24 & 7.6 & 0.022 & 4.24 & 7.9 & 0.022 & 4.24 & 8.6 & 0.023 & 4.23 & 8.2 & 0.022 & 4.25 & 8.3 & 0.019 \\
\hline$S_{3}$ & 4.60 & 53.1 & 0.038 & 4.59 & 53.1 & 0.037 & 4.57 & 52.9 & 0.038 & 4.56 & 53.2 & 0.037 & 4.55 & 52.2 & 0.037 & 4.55 & 53.2 & 0.038 & 4.57 & 55.6 & 0.033 \\
\hline$S_{4}$ & 4.64 & 1.1 & 0.031 & 4.64 & 1.1 & 0.031 & 4.64 & 1.3 & 0.029 & 4.64 & 1.3 & 0.030 & 4.65 & 1.4 & 0.028 & 4.63 & 1.4 & 0.022 & 4.63 & 2.4 & 0.021 \\
\hline$S_{5}$ & 5.34 & 39.3 & 0.033 & 5.24 & 11.0 & 0.016 & 5.18 & 9.5 & 0.014 & 5.21 & 13.3 & 0.016 & 5.00 & 4.8 & 0.049 & 4.95 & 3.2 & 0.015 & 5.02 & 14.3 & 0.173 \\
\hline$S_{6}$ & 5.53 & 98.8 & 0.030 & 5.36 & 21.7 & 0.008 & 5.31 & 21.3 & 0.009 & 5.33 & 20.5 & 0.008 & 5.05 & 19.4 & 0.174 & 5.02 & 21.3 & 0.206 & 5.07 & 10.7 & 0.048 \\
\hline$S_{7}$ & 5.56 & 10.5 & 0.008 & 5.44 & 4.7 & 0.014 & 5.39 & 4.6 & 0.011 & 5.41 & 2.3 & 0.009 & 5.18 & 9.9 & 0.013 & 5.16 & 13.6 & 0.016 & 5.25 & 14.3 & 0.018 \\
\hline$S_{8}$ & 5.61 & 4.0 & 0.006 & 5.53 & 108.1 & 0.032 & 5.52 & 101.9 & 0.031 & 5.52 & 2.8 & 0.021 & 5.29 & 20.4 & 0.009 & 5.28 & 18.3 & 0.006 & 5.37 & 26.1 & 0.009 \\
\hline$S_{9}$ & 5.68 & 32.3 & 0.068 & 5.58 & 0.4 & 0.007 & 5.54 & 4.4 & 0.010 & 5.52 & 95.4 & 0.023 & 5.36 & 3.4 & 0.008 & 5.36 & 2.2 & 0.011 & 5.40 & 6.2 & 0.018 \\
\hline$S_{10}$ & 5.78 & 1.7 & 0.006 & 5.60 & 12.5 & 0.002 & 5.56 & 7.3 & 0.001 & 5.55 & 19.7 & 0.003 & 5.46 & 3.6 & 0.005 & 5.46 & 21.7 & 0.051 & 5.46 & 69.4 & 0.055 \\
\hline$S_{11}$ & & & & 5.61 & 8.6 & 0.015 & 5.60 & 3.6 & 0.004 & 5.60 & 4.1 & 0.006 & 5.49 & 7.5 & 0.009 & 5.47 & 77.3 & 0.010 & 5.55 & 34.2 & 0.012 \\
\hline$S_{12}$ & & & & 5.68 & 23.4 & 0.058 & 5.67 & 30.9 & 0.065 & 5.66 & 28.6 & 0.060 & 5.51 & 95.8 & 0.032 & 5.53 & 2.5 & 0.002 & 5.58 & 1.1 & 0.001 \\
\hline$S_{13}$ & & & & 5.77 & 1.1 & 0.007 & 5.76 & 2.3 & 0.009 & 5.75 & 1.5 & 0.011 & 5.54 & 12.6 & 0.006 & 5.55 & 1.1 & 0.001 & 5.59 & 31.8 & 0.022 \\
\hline$S_{14}$ & & & & & & & 5.80 & 1.7 & 0.002 & 5.81 & 2.1 & 0.003 & 5.60 & 4.8 & 0.006 & 5.59 & 33.2 & 0.020 & 5.63 & 4.8 & 0.018 \\
\hline$S_{15}$ & & & & & & & 5.94 & 5.2 & 0.003 & 5.95 & 8.6 & 0.004 & 5.65 & 35.6 & 0.061 & 5.62 & 16.8 & 0.034 & 5.68 & 5.1 & 0.000 \\
\hline & & int4 (144 & & & & & & & & & & & & & & & & & & & \\
\hline State & $\Delta E$ & $\sigma^{T P A}$ & $f$ & & & & & & & & & & & & & & & & & & \\
\hline$S_{1}$ & 3.22 & 0.9 & 0.506 & & & & & & & & & & & & & & & & & & \\
\hline$S_{2}$ & 4.24 & 9.4 & 0.024 & & & & & & & & & & & & & & & & & & \\
\hline$S_{3}$ & 4.54 & 52.1 & 0.037 & & & & & & & & & & & & & & & & & & \\
\hline$S_{4}$ & 4.63 & 1.7 & 0.031 & & & & & & & & & & & & & & & & & & \\
\hline$S_{5}$ & 5.17 & 15.0 & 0.213 & & & & & & & & & & & & & & & & & & \\
\hline$S_{6}$ & 5.24 & 9.9 & 0.016 & & & & & & & & & & & & & & & & & & \\
\hline$S_{7}$ & 5.27 & 17.3 & 0.019 & & & & & & & & & & & & & & & & & & \\
\hline$S_{8}$ & 5.38 & 27.7 & 0.012 & & & & & & & & & & & & & & & & & & \\
\hline$S_{9}$ & 5.43 & 1.4 & 0.006 & & & & & & & & & & & & & & & & & & \\
\hline$S_{10}$ & 5.51 & 113.5 & 0.030 & & & & & & & & & & & & & & & & & & \\
\hline$S_{11}$ & 5.56 & 8.1 & 0.014 & & & & & & & & & & & & & & & & & & \\
\hline$S_{12}$ & 5.59 & 5.5 & 0.001 & & & & & & & & & & & & & & & & & & \\
\hline$S_{13}$ & 5.60 & 2.2 & 0.002 & & & & & & & & & & & & & & & & & & \\
\hline$S_{14}$ & 5.61 & 21.7 & 0.029 & & & & & & & & & & & & & & & & & & \\
\hline$S_{15}$ & 5.65 & 0.3 & 0.020 & & & & & & & & & & & & & & & & & & \\
\hline
\end{tabular}


Table S13: Impact of QM cluster size on excitation energy $(\Delta E[\mathrm{eV}])$, two-photon absorption cross-section $\left(\sigma^{T P A}[\mathrm{GM}]\right)$ and one-photon oscillator strength $(f)$ for avGFP-a. For composition of clusters, see Tables S4-S6. Number of atoms is given in parentheses.

\begin{tabular}{|c|c|c|c|c|c|c|c|c|c|c|c|c|c|c|c|c|c|c|c|c|c|}
\hline \multirow[b]{2}{*}{ State } & \multicolumn{3}{|c|}{0.00 (39) } & \multicolumn{3}{|c|}{ int1 (69) } & \multicolumn{3}{|c|}{ int2 $(8$} & & & \multicolumn{3}{|c|}{$25(114)$} & \multicolumn{3}{|c|}{ 0.30-noh } \\
\hline & $\Delta E$ & & $f$ & $\Delta E$ & $\sigma^{T P A}$ & $f$ & $\Delta E$ & $\sigma^{T P A}$ & $f$ & $\Delta E$ & $\sigma^{T P A}$ & $f$ & $\Delta E$ & & $f$ & $\Delta E$ & & $f$ & $\Delta E$ & & $f$ \\
\hline$S_{1}$ & 3.00 & 2.4 & 1.060 & 2.30 & 4.9 & 0.006 & 2.54 & 3.7 & 0.009 & 2.94 & 4.9 & 0.575 & 2.88 & 3.8 & 0.082 & 2.95 & 5.1 & 0.743 & 2.95 & 2.4 & 1.118 \\
\hline$S_{2}$ & 3.22 & 0.7 & 0.002 & 2.82 & 8.2 & 0.020 & 3.00 & 0.9 & 0.929 & 3.01 & 0.9 & 0.576 & 2.99 & 0.3 & 0.943 & 3.02 & 0.9 & 0.444 & 3.46 & 4.4 & 0.003 \\
\hline$S_{3}$ & 3.56 & 1.0 & 0.001 & 2.93 & 0.1 & 0.000 & 3.06 & 7.4 & 0.113 & 3.50 & 8.4 & 0.006 & 3.41 & 2.7 & 0.003 & 3.54 & 8.6 & 0.005 & 3.55 & 0.5 & 0.000 \\
\hline$S_{4}$ & 83 & 1.8 & 0.000 & 3.01 & 0.3 & 1.018 & 3.26 & 0.1 & 0.001 & 3.57 & 0.1 & 0.000 & 3.61 & 0.4 & 0.001 & 3.60 & 0.1 & 0.000 & 3.98 & 0.0 & 0.000 \\
\hline$S_{5}$ & .87 & 0.3 & 0.002 & 3.26 & 1.7 & 0.004 & 3.48 & 1.3 & 0.003 & 3.65 & 0.5 & 0.000 & 3.75 & 0.0 & 0.000 & 3.68 & 0.5 & 0.000 & 4.01 & 3.6 & 0.002 \\
\hline$S_{6}$ & 4.03 & 0.0 & 0.001 & 3.51 & 0.4 & 0.002 & 3.77 & 0.3 & 0.001 & 3.75 & 0.2 & 0.000 & 3.78 & 3.2 & 0.004 & 3.84 & 0.4 & 0.000 & 4.02 & 0.2 & 0.000 \\
\hline$S_{7}$ & 11 & 2.1 & 0.003 & 3.74 & 0.2 & 0.001 & 3.87 & 0.0 & 0.000 & 3.94 & 0.7 & 0.002 & 3.93 & 0.2 & 0.000 & 3.96 & 0.9 & 0.002 & 4.23 & 0.9 & 0.000 \\
\hline$S_{8}$ & 4.18 & 1.4 & 0.042 & 3.78 & 0.0 & 0.000 & 3.98 & 0.5 & 0.001 & 4.02 & 0.0 & 0.000 & 4.21 & 1.2 & 0.003 & 4.08 & 0.0 & 0.000 & 4.27 & 2.6 & 0.001 \\
\hline$S_{9}$ & 4.23 & 5.6 & 0.028 & 3.97 & 1.6 & 0.002 & 4.14 & 0.4 & 0.000 & 4.19 & 0.0 & 0.000 & 4.34 & 1.8 & 0.009 & 4.22 & 0.2 & 0.001 & 4.36 & 3.8 & 0.004 \\
\hline$S_{10}$ & 31 & 23.0 & 0.004 & 4.08 & 0.4 & 0.003 & 4.24 & 1.2 & 0.003 & 4.25 & 2.2 & 0.000 & 4.40 & 2.2 & 0.000 & 4.26 & 0.0 & 0.000 & 4.39 & 0.0 & 0.000 \\
\hline$S_{11}$ & 4.35 & 0.4 & 0.002 & 11 & 1.0 & 0.001 & 4.29 & 1.0 & 0.006 & 4.28 & 0.6 & 0.001 & 4.44 & 1.4 & 0.000 & 4.30 & 3.0 & 0.001 & 4.40 & 0.3 & 0.000 \\
\hline$S_{12}$ & 48 & 18.8 & 0.006 & 4.19 & 5.8 & 0.000 & 4.32 & 2.9 & 0.024 & 4.36 & 1.3 & 0.007 & 4.50 & 4.3 & 0.007 & 4.40 & 0.9 & 0.003 & 4.42 & 0.0 & 0.000 \\
\hline$S_{13}$ & & 16.6 & 0.011 & .28 & 0.5 & 0.000 & 4.40 & 7.1 & 0.001 & 4.43 & 1.0 & 0.001 & 4.59 & 0.9 & 0.008 & 4.51 & 3. & 0.003 & 4.54 & 1.0 & 0.001 \\
\hline$S_{14}$ & 4.70 & 15.9 & 0.014 & 30 & 6.0 & 0.044 & 4.42 & 1.3 & 0.001 & 4.49 & 0.0 & 0.000 & 4.61 & 20.6 & 0.052 & 4.55 & 0.0 & 0.000 & 4.56 & 0.0 & 0.000 \\
\hline$S_{15}$ & .72 & 5.0 & 0.031 & & 8. & 0.001 & 52 & 0. & 0.001 & 4.59 & 11.4 & 0.005 & 4.66 & 8.2 & 0.004 & 4.62 & 0.1 & 0.000 & 4.64 & 56.0 & 0.006 \\
\hline$S_{16}$ & & & 10 & & 6. & 0.001 & 59 & 10.3 & 0.005 & 4.63 & 42.6 & 0.014 & 4.79 & 14. & 0.001 & 4.63 & 20.4 & 0.006 & 4.76 & 13 & 0.080 \\
\hline$S_{17}$ & 4.84 & 16.1 & 0.006 & & 1.1 & 0.001 & 4.63 & 7.0 & 0.003 & 4.66 & 0.1 & 0.000 & 4.84 & 12.4 & 0.029 & 4.64 & 38.7 & 0.007 & 4.81 & & 0.002 \\
\hline$S_{18}$ & 4.99 & 0.4 & 0.016 & & 21.6 & 0.001 & 4.66 & 13.3 & 0. & 4.70 & 1. & 0.006 & 4.86 & 37 & 0.025 & 4.68 & 0.9 & 0.000 & 4.82 & & 0.001 \\
\hline$S_{19}$ & & 2.4 & 012 & 66 & 40.5 & 0.040 & 4.74 & 2.2 & 0.002 & 4.74 & 1.4 & 0.001 & 4.92 & 19.2 & 0.051 & 4.78 & 3.3 & 0.025 & 4.84 & & 0.000 \\
\hline$S_{20}$ & 01 & 28.4 & 0.048 & 4.69 & 13.9 & 0.006 & 4.84 & 2.0 & 0.001 & 4.82 & 11.4 & 0.089 & 4.94 & 3.0 & 0.003 & 4.82 & 12.5 & 0.080 & 4.94 & & 0.000 \\
\hline$S_{21}$ & .05 & 45.9 & 0.011 & 4. & 13.8 & 0.002 & & 9. & 0.039 & & 2. & 0.004 & 4.96 & 7 & 0.003 & 4.86 & 0.9 & 0.002 & 4.96 & & 0.063 \\
\hline$S_{22}$ & & 1. & 008 & & 0.5 & 000 & 4.92 & 39. & 0.011 & 4.89 & 9 & 0.006 & 4.98 & 5. & 0.001 & 4.91 & 3.7 & 0.004 & 4.97 & 7.0 & 0.008 \\
\hline$S_{23}$ & & 49.7 & 008 & & 53.8 & 029 & 4.95 & 4. & 0.001 & 4.91 & 7.0 & 0.003 & 5.05 & 1. & 0.001 & 4.93 & 10.4 & 0.001 & 5.02 & 0.0 & 0.000 \\
\hline$S_{24}$ & 5.25 & 2.7 & 0.004 & 82 & 13.5 & 0.013 & 98 & 17.3 & 0.058 & 4.94 & 0. & 0.000 & 5.16 & 8.3 & 0.003 & 5.01 & 0.1 & 0.000 & 5.05 & 1. & 0.000 \\
\hline$S_{25}$ & 5.37 & 26.2 & 0.002 & 4.88 & 6.5 & 0.007 & 5.03 & 20.4 & 0.001 & 5.03 & 8.5 & 0.009 & 5.19 & 12.6 & 0.011 & 5.06 & 3.8 & 0.006 & 5.11 & 8.1 & 0.002 \\
\hline- & & & & Con & nued on & ge S31 & Cont & lued on & ge S31 & Cont & fued on & ge $S 31$ & & & & & & & & & \\
\hline & & int4 (1 & & & 0.30 & & & int5 & & & int6 & & & int7 ( & & & 0.35 & & & int8 & \\
\hline State & $\Delta E$ & $\sigma^{T P}$ & $f$ & $\Delta E$ & & $f$ & $\Delta E$ & & $f$ & $\Delta E$ & & $f$ & $\Delta E$ & & $f$ & $\Delta E$ & & $f$ & $\Delta E$ & & $f$ \\
\hline$S_{1}$ & 2.95 & 2.8 & 1.041 & 2.93 & 1.7 & 0.966 & 2.95 & 2.8 & 1.022 & 2.94 & 2.7 & 0.999 & 2.94 & 2.6 & 0.991 & 2.93 & 1.8 & 0.961 & 2.94 & 2.6 & 0.992 \\
\hline$S_{2}$ & 3.50 & 4 & 0.003 & & 3.5 & 002 & 3.56 & & 0.003 & 3.51 & & 0.003 & 3.56 & 4.1 & 0.002 & 3.47 & 3. & 0.003 & 3.67 & 3. & 0.002 \\
\hline$S_{3}$ & & & & & & 0.000 & 3.66 & & 0.000 & 3.7 & & 0.000 & 3.72 & & 0.000 & 3.72 & & 0.000 & 3.72 & & 0.000 \\
\hline$S_{4}$ & .04 & & & & & & & & & & & 0.001 & 4.11 & 3 & 0.002 & 4.0 & & 0.0 & 4.20 & & 0.000 \\
\hline$S_{5}$ & 4.15 & 0 & 000 & & 0 & 0.000 & 4.14 & & 0.000 & 4.16 & & 0.000 & 4.19 & 0. & 0.000 & 4.15 & 0. & 0.000 & 4.22 & 3.7 & 0.002 \\
\hline$S_{6}$ & 4.17 & 0. & 000 & & & 0.000 & 4.18 & & 0.000 & 4.1 & & 0.000 & 4.21 & 0 & 0.000 & 4.15 & 0. & 0.000 & 4.30 & & 0.000 \\
\hline$S_{7}$ & & & & & & & & & & & & 0.001 & 4.30 & & 0.000 & 4.2 & & 0.000 & 4.33 & & 0.000 \\
\hline$S_{8}$ & 4.30 & 3 & & 4.26 & 2 & & 4.34 & 2. & 0.001 & 4.30 & 4.0 & 0.000 & 4.34 & 2.6 & 0.001 & 4.25 & 3. & 0.003 & 4.37 & 4.6 & 0.004 \\
\hline$S_{9}$ & 38 & 2. & 03 & & 2 & 03 & 4. & & 0.004 & 4.3 & & 0.004 & 4.42 & 2.4 & 0.004 & 4.40 & 2. & 0.004 & 4.47 & 0.7 & 0.001 \\
\hline$S_{10}$ & & & & & & & & & & & & & & & & & & & 4.50 & & 0.002 \\
\hline$S_{11}$ & 4.51 & 0. & 000 & & 0 & 000 & 4.57 & 0.0 & 0.000 & 4.53 & 0.0 & 0.000 & 4.61 & 0.0 & 0.000 & 4.52 & 0.0 & 0.000 & 4.63 & 61.2 & 0.011 \\
\hline$S_{12}$ & & 1. & & & & & 4.6 & & & 4. & 2 & 0.001 & 4.63 & 56.6 & 0.011 & 4.61 & 53.7 & 0.013 & 4.74 & 0.0 & 0.000 \\
\hline$S_{13}$ & & & & & & & & & & & & & & 0.0 & & & 0.3 & & 4.76 & 0.4 & 0.002 \\
\hline$S_{14}$ & 4.63 & 63.2 & 0.008 & 4.66 & 0.0 & 0.000 & 4.64 & 13.0 & 0.001 & 4.62 & 60.5 & 0.008 & 4.64 & 5.3 & 0.000 & 4.66 & 1.4 & 0.001 & 4.76 & 1.1 & 0.005 \\
\hline$S_{15}$ & .73 & 0.0 & 0.000 & 4.67 & 4.6 & 0.001 & 4.7 & 0.0 & 0.000 & 4.7 & 0. & 0.000 & 4.79 & 0. & 0.001 & 4.68 & 0.0 & 0.000 & 4.79 & 14.5 & 0.071 \\
\hline$S_{16}$ & & 11.3 & & & 15.1 & & 4.7 & 14.4 & & 4.7 & 11 & 0.075 & 4.79 & 14.2 & 0.079 & 4.75 & 14.1 & 0.078 & 4.84 & 1.7 & 0.001 \\
\hline$S_{17}$ & 4.83 & 4.9 & 0.001 & 4.87 & 2.3 & 0.012 & 4.86 & 2.5 & 0.002 & 4.85 & 5.1 & 0.001 & 4.87 & 2.8 & 0.002 & 4.86 & 3.2 & 0.019 & 4.89 & 0.0 & 0.000 \\
\hline
\end{tabular}




\begin{tabular}{|c|c|c|c|c|c|c|c|c|c|c|c|c|c|c|c|c|c|c|c|c|c|}
\hline$S_{18}$ & 4.89 & 5.9 & 0.018 & 4.92 & 1.4 & 0.002 & 4.93 & 5.9 & 0.012 & 4.90 & 4.9 & 0.017 & 4.92 & 5.5 & 0.014 & 4.91 & 1.5 & 0.001 & 4.93 & 6.2 & 0.018 \\
\hline$S_{19}$ & 4.94 & 0.0 & 0.001 & 4.95 & 0.1 & 0.004 & 4.95 & 0.1 & 0.003 & 4.96 & 0.0 & 0.044 & 4.96 & 0.0 & 0.053 & 4.96 & 0.0 & 0.053 & 4.96 & 0.0 & 0.053 \\
\hline$S_{20}$ & 4.96 & 0.0 & 0.056 & 4.96 & 0.0 & 0.056 & 4.96 & 0.0 & 0.055 & 4.96 & 0.0 & 0.010 & 5.02 & 0.0 & 0.000 & 4.98 & 0.0 & 0.000 & 5.08 & 2.8 & 0.003 \\
\hline & \multicolumn{3}{|c|}{ int9 (260) } & \multicolumn{3}{|c|}{$0.40(284)$} & \multicolumn{3}{|c|}{$0.45(313)$} & \multicolumn{3}{|c|}{$0.50(342)$} & & & & & & & & & \\
\hline State & $\Delta E$ & $\sigma^{T P A}$ & $f$ & $\Delta E$ & $\sigma^{T P A}$ & $f$ & $\Delta E$ & $\sigma^{T P A}$ & $f$ & $\Delta E$ & $\sigma^{T P A}$ & $f$ & & & & & & & & & \\
\hline$S_{2}$ & 3.57 & 3.3 & 0.002 & 3.68 & 2.9 & 0.001 & 3.85 & 0.5 & 0.000 & 3.98 & 0.5 & 0.000 & & & & & & & & & \\
\hline$S_{3}$ & 3.79 & 0.5 & 0.000 & 3.78 & 0.5 & 0.000 & 4.07 & 0.3 & 0.000 & 4.13 & 0.1 & 0.000 & & & & & & & & & \\
\hline$S_{4}$ & 4.15 & 3.4 & 0.002 & 4.23 & 0.1 & 0.000 & 4.29 & 1.7 & 0.001 & 4.30 & 3.7 & 0.000 & & & & & & & & & \\
\hline$S_{5}$ & 4.23 & 0.1 & 0.000 & 4.26 & 5.2 & 0.004 & 4.30 & 2.8 & 0.000 & 4.36 & 0.6 & 0.000 & & & & & & & & & \\
\hline$S_{6}$ & 4.29 & 3.5 & 0.001 & 4.29 & 2.7 & 0.001 & 4.50 & 1.2 & 0.002 & & & & & & & & & & & & \\
\hline$S_{8}$ & 4.34 & 1.4 & 0.001 & 4.42 & 0.0 & 0.000 & 4.63 & 5.2 & 0.001 & & & & & & & & & & & & \\
\hline$S_{9}$ & 4.44 & 1.6 & 0.003 & 4.47 & 0.7 & 0.001 & 4.65 & 27.9 & 0.011 & & & & & & & & & & & & \\
\hline$S_{10}$ & 4.47 & 0.6 & 0.001 & 4.52 & 0.5 & 0.001 & 4.71 & 5.2 & 0.002 & & & & & & & & & & & & \\
\hline$S_{11}$ & 4.63 & 57.3 & 0.018 & 4.63 & 58.0 & 0.020 & 4.80 & 18.6 & 0.080 & & & & & & & & & & & & \\
\hline$S_{12}$ & 4.67 & 0.0 & 0.000 & 4.79 & 16.1 & 0.073 & 4.88 & 1.1 & 0.001 & & & & & & & & & & & & \\
\hline$S_{13}$ & 4.74 & 0.0 & 0.000 & 4.81 & 0.0 & 0.000 & 4.90 & 0.0 & 0.000 & & & & & & & & & & & & \\
\hline$S_{14}$ & 4.75 & 0.9 & 0.001 & 4.84 & 1.8 & 0.005 & 4.96 & 0.1 & 0.054 & & & & & & & & & & & & \\
\hline$S_{15}$ & 4.79 & 16.2 & 0.076 & 4.86 & 0.2 & 0.001 & 4.97 & 37.7 & 0.013 & & & & & & & & & & & & \\
\hline$S_{16}$ & 4.85 & 0.0 & 0.000 & 4.86 & 1.1 & 0.004 & & & & & & & & & & & & & & & \\
\hline$S_{17}$ & 4.87 & 1.7 & 0.008 & 4.93 & 8.3 & 0.012 & & & & & & & & & & & & & & & \\
\hline$S_{20}$ & 5.01 & 0.8 & 0.001 & 5.04 & 3.0 & 0.001 & & & & & & & & & & & & & & & \\
\hline
\end{tabular}


Table S13 continued for the int 1, int2 and 0.20 QM clusters.

\begin{tabular}{|c|c|c|c|c|c|c|c|c|c|}
\hline \multirow[b]{2}{*}{ State } & \multicolumn{3}{|c|}{ int1 (69) } & \multicolumn{3}{|c|}{ int2 (81) } & \multicolumn{3}{|c|}{$0.20(96)$} \\
\hline & $\Delta E$ & $\sigma^{T P A}$ & $f$ & $\Delta E$ & $\sigma^{T P A}$ & $f$ & $\Delta E$ & $\sigma^{T P A}$ & $f$ \\
\hline$S_{26}$ & 4.90 & 6.1 & 0.025 & 5.04 & 91.1 & 0.001 & 5.04 & 0.9 & 0.000 \\
\hline$S_{27}$ & 4.92 & 7.5 & 0.012 & 5.08 & 854.7 & 0.017 & 5.10 & 4.4 & 0.001 \\
\hline$S_{28}$ & 4.92 & 16.7 & 0.028 & 5.10 & 30.5 & 0.008 & 5.17 & 15.5 & 0.004 \\
\hline$S_{29}$ & 4.95 & 1.4 & 0.017 & 5.11 & 40.8 & 0.001 & 5.20 & 0.2 & 0.000 \\
\hline$S_{30}$ & 4.98 & 4.6 & 0.000 & 5.15 & 8.5 & 0.011 & 5.23 & 38.2 & 0.029 \\
\hline$S_{31}$ & 5.01 & 2.5 & 0.000 & 5.16 & 26.2 & 0.002 & 5.23 & 52.9 & 0.039 \\
\hline$S_{32}$ & 5.01 & 0.5 & 0.000 & 5.18 & 6.2 & 0.002 & 5.24 & 0.2 & 0.001 \\
\hline$S_{33}$ & 5.03 & 0.9 & 0.001 & 5.27 & 26.1 & 0.006 & 5.28 & 0.1 & 0.000 \\
\hline$S_{34}$ & 5.08 & 8.8 & 0.001 & 5.28 & 12.9 & 0.001 & 5.32 & 25.6 & 0.003 \\
\hline$S_{35}$ & 5.13 & 9.8 & 0.003 & 5.32 & 0.6 & 0.000 & 5.34 & 1.3 & 0.001 \\
\hline$S_{36}$ & 5.20 & 0.2 & 0.000 & 5.36 & 63.6 & 0.002 & 5.35 & 203.3 & 0.001 \\
\hline$S_{37}$ & 5.21 & 17.0 & 0.018 & 5.38 & 62.1 & 0.004 & 5.38 & 22.1 & 0.000 \\
\hline$S_{38}$ & 5.26 & 34.4 & 0.001 & 5.44 & 157.3 & 0.001 & 5.38 & 5.4 & 0.000 \\
\hline$S_{39}$ & 5.30 & 40.1 & 0.001 & 5.47 & 2.1 & 0.000 & 5.39 & 104.8 & 0.002 \\
\hline$S_{40}$ & 5.30 & 0.2 & 0.001 & 5.47 & 7.4 & 0.004 & 5.39 & 38.7 & 0.005 \\
\hline$S_{41}$ & 5.33 & 7.0 & 0.003 & 5.50 & 37.8 & 0.002 & & & \\
\hline$S_{42}$ & 5.35 & 6.9 & 0.006 & 5.54 & 111.2 & 0.002 & & & \\
\hline$S_{43}$ & 5.39 & 3.3 & 0.003 & 5.57 & 76.0 & 0.003 & & & \\
\hline$S_{44}$ & 5.40 & 14.5 & 0.001 & 5.58 & 665.1 & 0.001 & & & \\
\hline$S_{45}$ & 5.42 & 104.7 & 0.001 & 5.60 & 3101.4 & 0.002 & & & \\
\hline$S_{46}$ & 5.45 & 844.8 & 0.003 & 5.60 & 5.5 & 0.001 & & & \\
\hline$S_{47}$ & 5.50 & 4.5 & 0.000 & 5.63 & 2470.0 & 0.009 & & & \\
\hline$S_{48}$ & 5.52 & 475.0 & 0.004 & 5.64 & 1137.5 & 0.001 & & & \\
\hline$S_{49}$ & 5.56 & 52.7 & 0.003 & 5.68 & 758.3 & 0.001 & & & \\
\hline$S_{50}$ & 5.60 & 1720.3 & 0.001 & 5.69 & 319.2 & 0.000 & & & \\
\hline
\end{tabular}


Table S14: Impact of QM cluster size on excitation energy $(\Delta E[\mathrm{eV}])$, two-photon absorption cross-section $\left(\sigma^{T P A}[\mathrm{GM}]\right)$ and one-photon oscillator strength $(f)$ for avGFP-a. For composition of clusters, see Tables S4-S6. Number of atoms is given in parentheses. The MM subsystem is described by electrostatic embedding.

\begin{tabular}{|c|c|c|c|c|c|c|c|c|c|c|c|c|c|c|c|c|c|c|c|c|c|}
\hline \multirow[b]{2}{*}{ State } & \multicolumn{3}{|c|}{$0.00(39)$} & \multicolumn{3}{|c|}{ int1 (69) } & \multicolumn{3}{|c|}{ int2 (81) } & \multicolumn{3}{|c|}{$0.20(96)$} & \multicolumn{3}{|c|}{ int3 (111) } & \multicolumn{3}{|c|}{$0.25(114)$} & \multicolumn{3}{|c|}{ 0.30-noh (154) } \\
\hline & $\Delta E$ & $\sigma^{T P A}$ & $f$ & $\Delta E$ & $\sigma^{T P A}$ & $f$ & $\Delta E$ & $\sigma^{T P A}$ & $f$ & $\Delta E$ & $\sigma^{T P A}$ & $f$ & $\Delta E$ & $\sigma^{T P A}$ & $f$ & $\Delta E$ & $\sigma^{T P A}$ & $f$ & $\Delta E$ & $\sigma^{T P A}$ & $f$ \\
\hline$S_{1}$ & 3.10 & 3.1 & 1.064 & 3.09 & 2.9 & 1.013 & 3.07 & 2.9 & 1.015 & 3.04 & 5.3 & 1.130 & 3.04 & 2.8 & 0.975 & 3.03 & 5.7 & 1.167 & 3.00 & 5.2 & 1.089 \\
\hline$S_{2}$ & 4.15 & 1.2 & 0.002 & 4.17 & 0.6 & 0.003 & 4.15 & 0.6 & 0.003 & 4.23 & 1.0 & 0.003 & 4.32 & 3.1 & 0.015 & 4.20 & 0.8 & 0.003 & 4.32 & 5.9 & 0.009 \\
\hline$S_{3}$ & 4.35 & 1.9 & 0.018 & 4.34 & 2.8 & 0.019 & 4.34 & 2.8 & 0.014 & 4.34 & 4.9 & 0.007 & 4.49 & 0.9 & 0.001 & 4.34 & 5.5 & 0.012 & 4.48 & 0.4 & 0.000 \\
\hline$S_{4}$ & 4.55 & 0.3 & 0.003 & 4.55 & 0.4 & 0.003 & 4.53 & 0.4 & 0.004 & 4.35 & 0.6 & 0.007 & 4.53 & 0.5 & 0.003 & 4.43 & 1.6 & 0.001 & 4.50 & 3.3 & 0.001 \\
\hline$S_{5}$ & 4.62 & 48.4 & 0.021 & 4.60 & 48.0 & 0.015 & 4.61 & 49.6 & 0.014 & 4.58 & 88.0 & 0.007 & 4.60 & 49.7 & 0.013 & 4.58 & 95.1 & 0.006 & 4.57 & 89.5 & 0.006 \\
\hline$S_{6}$ & 4.87 & 1.4 & 0.008 & 4.77 & 0.3 & 0.001 & 4.71 & 0.2 & 0.001 & 4.70 & 0.5 & 0.007 & 4.68 & 0.6 & 0.001 & 4.69 & 0.6 & 0.008 & 4.63 & 0.2 & 0.000 \\
\hline$S_{7}$ & 4.92 & 0.5 & 0.001 & 4.82 & 1.0 & 0.001 & 4.80 & 1.1 & 0.002 & 4.87 & 0.7 & 0.012 & 4.75 & 0.7 & 0.001 & 4.82 & 0.7 & 0.006 & 4.68 & 1.0 & 0.002 \\
\hline$S_{8}$ & 4.95 & 4.3 & 0.104 & 4.92 & 0.7 & 0.001 & 4.92 & 8.5 & 0.076 & 4.90 & 1.6 & 0.005 & 4.83 & 1.9 & 0.001 & 4.88 & 1.9 & 0.010 & 4.71 & 1.0 & 0.008 \\
\hline$S_{9}$ & 5.00 & 2.8 & 0.001 & 4.95 & 8.9 & 0.104 & 4.94 & 3.1 & 0.013 & 4.92 & 4.5 & 0.013 & 4.92 & 9.3 & 0.075 & 4.90 & 4.7 & 0.001 & 4.77 & 1.4 & 0.000 \\
\hline$S_{11}$ & 5.28 & 15.2 & 0.043 & 4.99 & 1.5 & 0.002 & 4.95 & 0.2 & 0.003 & 5.03 & 0.4 & 0.004 & 4.94 & 0.3 & 0.002 & 5.01 & 1.2 & 0.006 & 4.91 & 0.9 & 0.022 \\
\hline$S_{12}$ & 5.33 & 22.8 & 0.005 & 5.18 & 2.9 & 0.012 & 5.12 & 2.1 & 0.004 & 5.16 & 0.3 & 0.002 & 5.06 & 0.9 & 0.009 & 5.09 & 0.2 & 0.002 & 4.93 & 6.1 & 0.032 \\
\hline$S_{13}$ & 5.39 & 32.1 & 0.013 & 5.24 & 1.6 & 0.011 & 5.20 & 0.3 & 0.009 & 5.20 & 2.6 & 0.003 & 5.11 & 0.7 & 0.002 & 5.17 & 2.8 & 0.003 & 5.01 & 0.3 & 0.049 \\
\hline$S_{14}$ & 5.51 & 20.0 & 0.000 & 5.27 & 7.5 & 0.001 & 5.24 & 4.3 & 0.007 & 5.26 & 10.0 & 0.007 & 5.20 & 4.5 & 0.012 & 5.22 & 9.6 & 0.008 & 5.07 & 0.6 & 0.001 \\
\hline$S_{15}$ & 5.54 & 88.3 & 0.003 & 5.31 & 28.9 & 0.036 & 5.27 & 26.9 & 0.038 & 5.27 & 5.6 & 0.011 & 5.23 & 23.9 & 0.024 & 5.24 & 0.9 & 0.006 & 5.10 & 3.4 & 0.004 \\
\hline & \multicolumn{3}{|c|}{ int4 (197) } & \multicolumn{3}{|c|}{$0.30(219)$} & \multicolumn{3}{|c|}{ int5 (220) } & \multicolumn{3}{|c|}{ int6 (225) } & \multicolumn{3}{|c|}{ int7 (245) } & \multicolumn{3}{|c|}{$0.35(254)$} & \multicolumn{3}{|c|}{ int8 (254) } \\
\hline State & $\Delta E$ & $\sigma^{T P A}$ & $f$ & $\Delta E$ & $\sigma^{T P A}$ & $f$ & $\Delta E$ & $\sigma^{T P A}$ & $f$ & $\Delta E$ & $\sigma^{T P A}$ & $f$ & $\Delta E$ & $\sigma^{T P A}$ & $f$ & $\Delta E$ & $\sigma^{T P A}$ & $f$ & $\Delta E$ & $\sigma^{T P A}$ & $f$ \\
\hline$S_{1}$ & 2.98 & 4.7 & 1.021 & 2.99 & 3.7 & 0.951 & 3.00 & 4.8 & 0.997 & 2.98 & 4.3 & 0.981 & 2.98 & 4.3 & 0.973 & 2.98 & 3.7 & 0.949 & 2.98 & 4.5 & 0.970 \\
\hline$S_{2}$ & 4.32 & 6.2 & 0.005 & 4.31 & 4.8 & 0.007 & 4.32 & 5.6 & 0.011 & 4.32 & 5.8 & 0.006 & 4.32 & 5.7 & 0.006 & 4.31 & 4.9 & 0.005 & 4.32 & 6.0 & 0.008 \\
\hline$S_{3}$ & 4.40 & 0.1 & 0.000 & 4.43 & 0.2 & 0.000 & 4.56 & 73.5 & 0.005 & 4.38 & 0.2 & 0.000 & 4.39 & 0.7 & 0.000 & 4.41 & 0.1 & 0.000 & 4.54 & 0.4 & 0.000 \\
\hline$S_{6}$ & 4.60 & 20.8 & 0.001 & 4.58 & 32.4 & 0.005 & 4.68 & 0.4 & 0.000 & 4.59 & 69.1 & 0.007 & 4.58 & 66.9 & 0.006 & 4.58 & 64.9 & 0.010 & 4.70 & 0.6 & 0.001 \\
\hline$S_{7}$ & 4.68 & 0.3 & 0.000 & 4.68 & 0.1 & 0.001 & 4.74 & 1.1 & 0.012 & 4.67 & 0.2 & 0.000 & 4.67 & 0.2 & 0.000 & 4.62 & 0.2 & 0.000 & 4.71 & 0.2 & 0.003 \\
\hline$S_{8}$ & 4.70 & 1.2 & 0.007 & 4.68 & 0.8 & 0.000 & 4.78 & 0.2 & 0.000 & 4.71 & 1.2 & 0.008 & 4.67 & 1.5 & 0.000 & 4.66 & 0.3 & 0.000 & 4.74 & 1.4 & 0.010 \\
\hline$S_{9}$ & 4.72 & 1.9 & 0.001 & 4.71 & 2.8 & 0.008 & 4.80 & 1.4 & 0.000 & 4.72 & 1.8 & 0.001 & 4.72 & 1.5 & 0.011 & 4.71 & 2.5 & 0.009 & 4.78 & 2.2 & 0.000 \\
\hline$S_{10}$ & 4.87 & 7.8 & 0.050 & 4.74 & 0.8 & 0.005 & 4.87 & 5.8 & 0.011 & 4.85 & 1.6 & 0.002 & 4.81 & 1.3 & 0.000 & 4.72 & 0.7 & 0.002 & 4.83 & 1.0 & 0.001 \\
\hline$S_{11}$ & 4.89 & 4.6 & 0.018 & 4.82 & 1.6 & 0.004 & 4.90 & 2.1 & 0.008 & 4.88 & 9.6 & 0.064 & 4.87 & 7.3 & 0.053 & 4.83 & 1.9 & 0.000 & 4.88 & 6.1 & 0.029 \\
\hline$S_{12}$ & 4.92 & 1.1 & 0.014 & 4.87 & 8.6 & 0.057 & 4.93 & 8.0 & 0.068 & 4.92 & 1.3 & 0.017 & 4.90 & 1.8 & 0.004 & 4.87 & 9.7 & 0.072 & 4.90 & 2.0 & 0.023 \\
\hline$S_{13}$ & 4.97 & 0.0 & 0.000 & 4.91 & 1.0 & 0.012 & 5.00 & 1.2 & 0.001 & 4.94 & 2.7 & 0.001 & 4.91 & 1.4 & 0.014 & 4.92 & 0.5 & 0.009 & 4.92 & 2.4 & 0.018 \\
\hline$S_{14}$ & 4.97 & 4.9 & 0.003 & 4.96 & 0.7 & 0.009 & 5.02 & 0.7 & 0.044 & 4.97 & 3.8 & 0.002 & 4.93 & 5.2 & 0.007 & 4.94 & 0.1 & 0.000 & 4.94 & 4.2 & 0.018 \\
\hline$S_{15}$ & 5.00 & 2.3 & 0.038 & 4.99 & 3.9 & 0.005 & 5.05 & 2.2 & 0.004 & 5.00 & 3.5 & 0.029 & 4.96 & 0.2 & 0.007 & 4.95 & 4.1 & 0.008 & 4.98 & 0.5 & 0.001 \\
\hline & & int9 (26 & & & $0.40(28$ & & & $0.45(31$ & & & $0.50(34$ & & & & & & & & & & \\
\hline State & $\Delta E$ & $\sigma^{T P A}$ & $f$ & $\Delta E$ & $\sigma^{T P A}$ & $f$ & $\Delta E$ & $\sigma^{T P A}$ & $f$ & $\Delta E$ & $\sigma^{T P A}$ & $f$ & & & & & & & & & \\
\hline$S_{1}$ & 2.98 & 3.7 & 0.927 & 2.98 & 3.8 & 0.922 & 2.98 & 4.0 & 0.926 & 2.98 & 4.0 & 0.914 & & & & & & & & & \\
\hline$S_{2}$ & 4.32 & 5.3 & 0.005 & 4.32 & 5.0 & 0.006 & 4.31 & 5.3 & 0.007 & 4.31 & 4.6 & 0.008 & & & & & & & & & \\
\hline$S_{3}$ & 4.40 & 0.2 & 0.000 & 4.42 & 0.3 & 0.000 & 4.51 & 0.5 & 0.000 & 4.36 & 0.8 & 0.000 & & & & & & & & & \\
\hline$S_{4}$ & 4.51 & 8.3 & 0.004 & 4.53 & 19.1 & 0.007 & 4.55 & 49.7 & 0.012 & 4.55 & 80.3 & 0.015 & & & & & & & & & \\
\hline$S_{5}$ & 4.52 & 1.1 & 0.000 & 4.55 & 2.3 & 0.001 & 4.58 & 34.5 & 0.004 & 4.59 & 0.8 & 0.001 & & & & & & & & & \\
\hline$S_{6}$ & 4.58 & 72.7 & 0.014 & 4.58 & 62.2 & 0.010 & 4.60 & 1.9 & 0.000 & & & & & & & & & & & & \\
\hline$S_{7}$ & 4.66 & 1.6 & 0.000 & 4.65 & 1.7 & 0.000 & 4.63 & 1.4 & 0.000 & & & & & & & & & & & & \\
\hline$S_{8}$ & 4.67 & 0.3 & 0.000 & 4.69 & 0.4 & 0.000 & 4.74 & 0.4 & 0.000 & & & & & & & & & & & & \\
\hline$S_{9}$ & 4.70 & 1.7 & 0.001 & 4.69 & 1.4 & 0.000 & 4.75 & 2.0 & 0.017 & & & & & & & & & & & & \\
\hline
\end{tabular}




\begin{tabular}{l|lll|lll|}
$S_{11}$ & 4.81 & 1.7 & 0.000 & 4.79 & 1.9 & 0.000 \\
$S_{12}$ & 4.86 & 7.3 & 0.050 & 4.86 & 4.8 & 0.033 \\
$S_{13}$ & 4.90 & 0.2 & 0.000 & 4.87 & 0.3 & 0.002 \\
$S_{14}$ & 4.91 & 1.2 & 0.005 & 4.90 & 2.2 & 0.002 \\
$S_{15}$ & 4.93 & 5.0 & 0.020 & 4.91 & 0.4 & 0.002 \\
\hline
\end{tabular}

Table S15: Impact of QM cluster size on excitation energy $(\Delta E[\mathrm{eV}])$, two-photon absorption cross-section $\left(\sigma^{T P A}[\mathrm{GM}]\right)$ and one-photon oscillator strength $(f)$ for avGFP-a. For composition of clusters, see Tables S4-S6. Number of atoms is given in parentheses. The MM subsystem is described by the polarizable embedding.

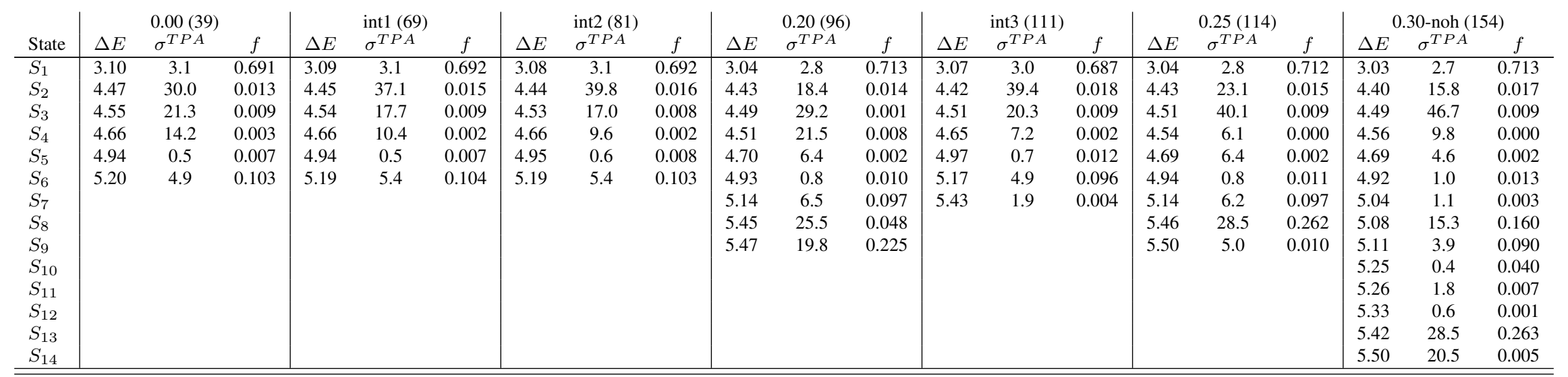


Table S16: Impact of QM cluster size on excitation energy $(\Delta E[\mathrm{eV}])$, two-photon absorption cross-section $\left(\sigma^{T P A}[\mathrm{GM}]\right)$ and one-photon oscillator strength $(f)$ for Citrine. For composition of clusters, see Tables S7-S9. Number of atoms is given in parentheses.

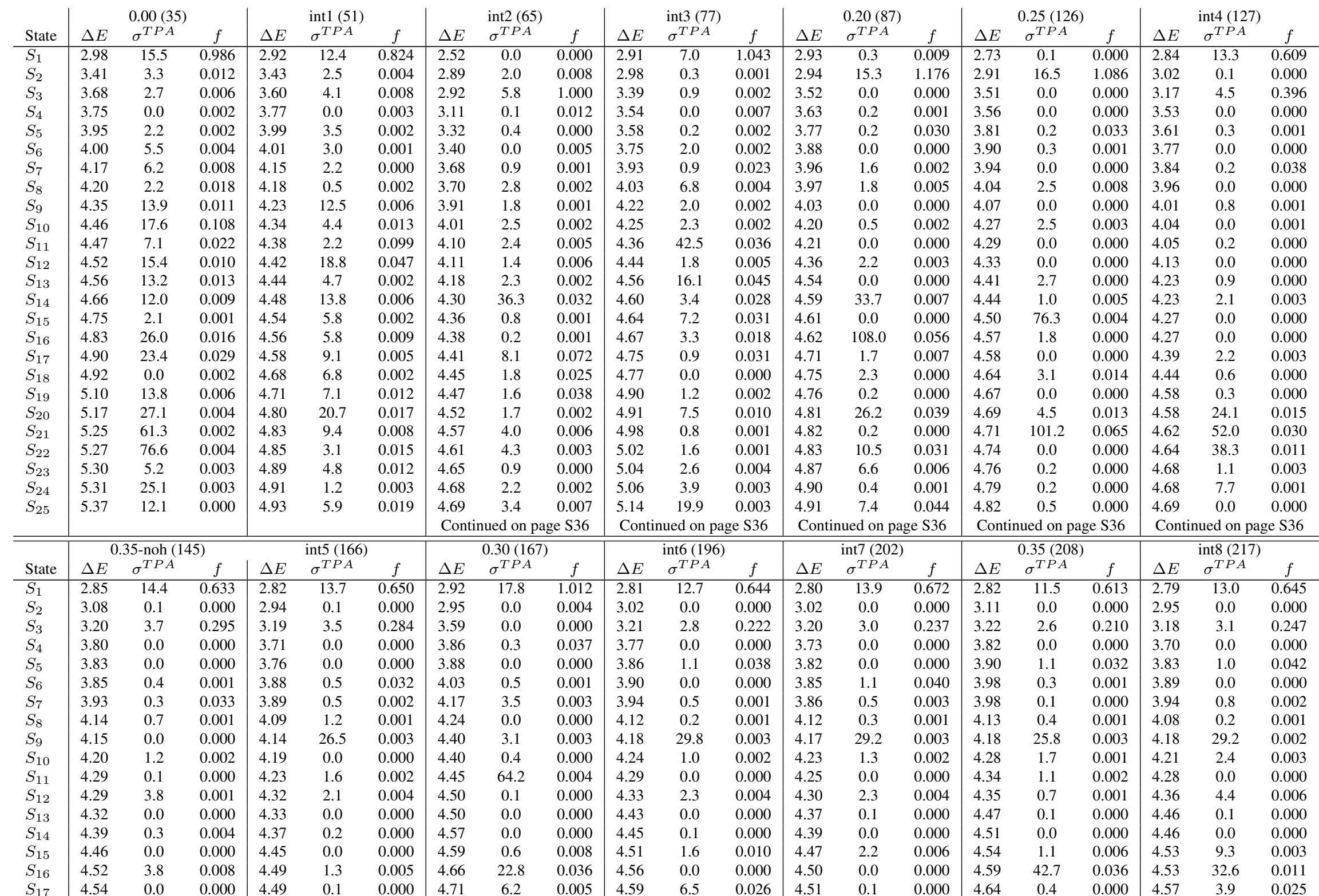




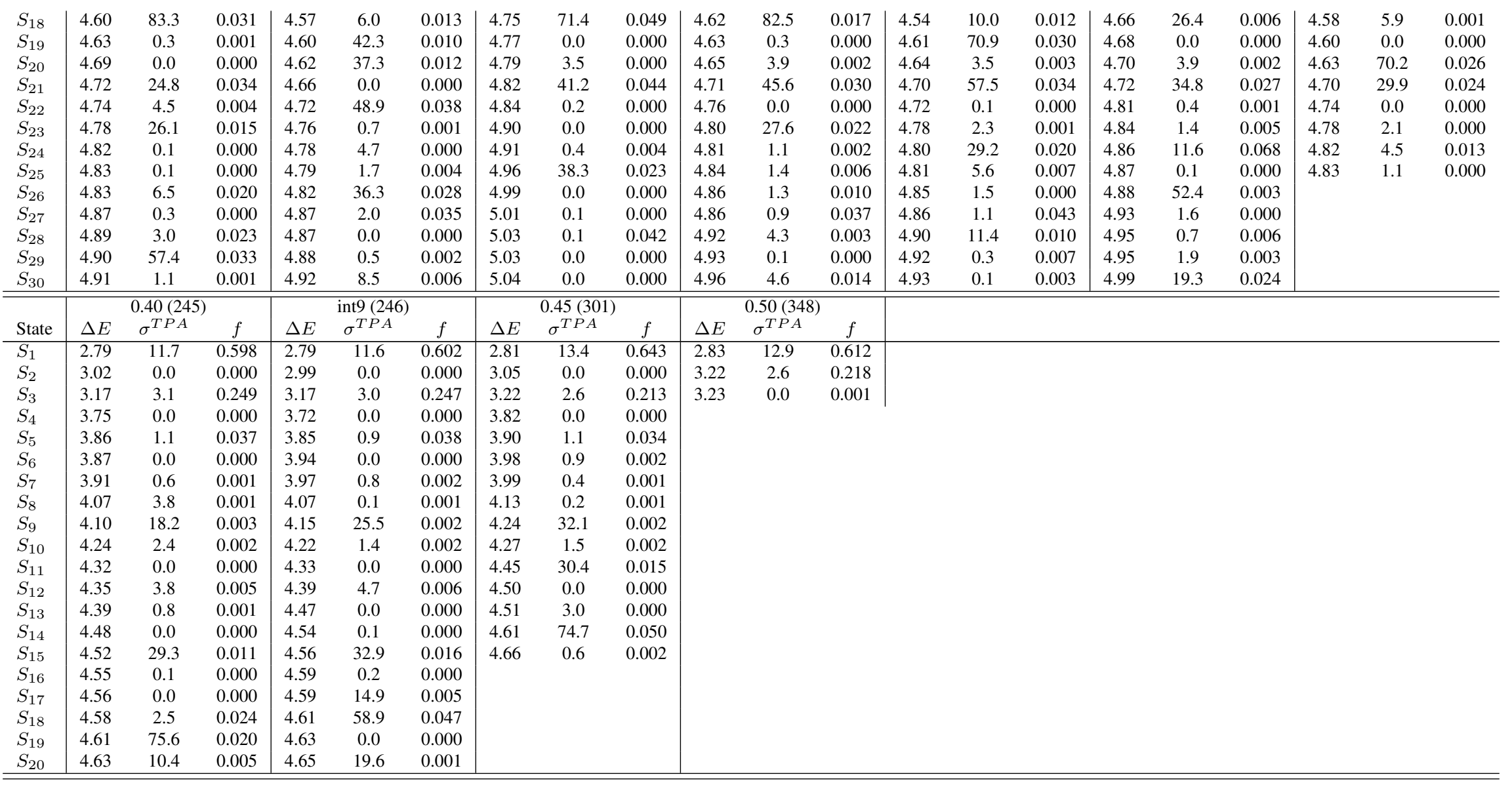


Table S16 continued for the int2, int3, 0.20, 0.25 and int4 QM clusters.

\begin{tabular}{|c|c|c|c|c|c|c|c|c|c|c|c|c|c|c|c|}
\hline \multirow[b]{2}{*}{ State } & \multicolumn{3}{|c|}{ int2 (65) } & \multicolumn{3}{|c|}{ int3 (77) } & \multicolumn{3}{|c|}{$0.20(87)$} & \multicolumn{3}{|c|}{0.25 (126) } & \multicolumn{3}{|c|}{ int4 (127) } \\
\hline & $\Delta E$ & $\sigma^{T P A}$ & $f$ & $\Delta E$ & $\sigma^{T P A}$ & $f$ & $\Delta E$ & $\sigma^{T P A}$ & $f$ & $\Delta E$ & $\sigma^{T P A}$ & $f$ & $\Delta E$ & $\sigma^{T P A}$ & $f$ \\
\hline$S_{26}$ & 4.74 & 1.6 & 0.005 & 5.19 & 1.2 & 0.001 & 4.94 & 2.8 & 0.001 & 4.82 & 27.6 & 0.070 & 4.73 & 0.0 & 0.000 \\
\hline$S_{27}$ & 4.77 & 0.4 & 0.001 & 5.21 & 5.7 & 0.002 & 5.00 & 0.4 & 0.001 & 4.89 & 0.0 & 0.000 & 4.76 & 46.7 & 0.028 \\
\hline$S_{28}$ & 4.81 & 10.2 & 0.032 & 5.30 & 4.7 & 0.001 & 5.01 & 0.2 & 0.001 & 4.95 & 0.1 & 0.001 & 4.80 & 0.0 & 0.000 \\
\hline$S_{29}$ & 4.87 & 0.6 & 0.003 & 5.33 & 6.4 & 0.000 & 5.04 & 0.8 & 0.004 & 4.96 & 6.0 & 0.002 & 4.82 & 2.6 & 0.004 \\
\hline$S_{30}$ & 4.88 & 4.4 & 0.003 & 5.36 & 194.9 & 0.005 & 5.09 & 0.0 & 0.000 & 4.96 & 0.1 & 0.000 & 4.85 & 6.8 & 0.002 \\
\hline$S_{31}$ & 4.94 & 0.1 & 0.000 & & & & 5.10 & 2.5 & 0.000 & 5.00 & 0.0 & 0.004 & 4.86 & 9.4 & 0.012 \\
\hline$S_{32}$ & 4.98 & 10.3 & 0.001 & & & & 5.17 & 15.7 & 0.008 & 5.03 & 0.5 & 0.002 & 4.89 & 4.9 & 0.037 \\
\hline$S_{33}$ & 5.03 & 6.5 & 0.003 & & & & 5.18 & 0.2 & 0.000 & 5.03 & 16.4 & 0.007 & 4.90 & 0.1 & 0.000 \\
\hline$S_{34}$ & 5.06 & 2.3 & 0.001 & & & & 5.21 & 0.9 & 0.003 & 5.03 & 0.1 & 0.041 & 4.91 & 0.5 & 0.002 \\
\hline$S_{35}$ & 5.08 & 2.7 & 0.002 & & & & 5.23 & 0.2 & 0.000 & 5.06 & 102.5 & 0.002 & 4.91 & 2.7 & 0.017 \\
\hline$S_{36}$ & 5.12 & 8.5 & 0.003 & & & & 5.25 & 7.4 & 0.002 & 5.10 & 0.5 & 0.003 & 4.92 & 0.0 & 0.001 \\
\hline$S_{37}$ & 5.13 & 1.4 & 0.000 & & & & 5.26 & 206.3 & 0.003 & 5.11 & 0.1 & 0.002 & 4.95 & 3.9 & 0.021 \\
\hline$S_{38}$ & 5.14 & 0.2 & 0.000 & & & & 5.30 & 1.1 & 0.006 & 5.14 & 0.3 & 0.002 & 4.98 & 0.0 & 0.000 \\
\hline$S_{39}$ & 5.19 & 1.0 & 0.000 & & & & 5.33 & 3.4 & 0.001 & 5.18 & 4.8 & 0.002 & 4.98 & 0.0 & 0.001 \\
\hline$S_{40}$ & 5.26 & 23.5 & 0.003 & & & & 5.34 & 0.2 & 0.000 & 5.20 & 0.0 & 0.000 & 5.03 & 0.1 & 0.046 \\
\hline$S_{41}$ & 5.27 & 13.5 & 0.001 & & & & & & & & & & 5.04 & 0.3 & 0.001 \\
\hline$S_{42}$ & 5.28 & 71.1 & 0.001 & & & & & & & & & & 5.04 & 2.9 & 0.000 \\
\hline$S_{43}$ & 5.29 & 167.7 & 0.003 & & & & & & & & & & 5.09 & 3.3 & 0.005 \\
\hline$S_{44}$ & 5.33 & 69.1 & 0.001 & & & & & & & & & & 5.09 & 0.1 & 0.000 \\
\hline$S_{45}$ & 5.36 & 3.6 & 0.000 & & & & & & & & & & 5.11 & 0.9 & 0.001 \\
\hline$S_{46}$ & 5.37 & 54.8 & 0.001 & & & & & & & & & & 5.13 & 0.1 & 0.000 \\
\hline$S_{47}$ & 5.39 & 72.9 & 0.001 & & & & & & & & & & 5.15 & 0.4 & 0.005 \\
\hline$S_{48}$ & 5.42 & 295.7 & 0.000 & & & & & & & & & & 5.21 & 0.1 & 0.000 \\
\hline$S_{49}$ & 5.44 & 301.6 & 0.003 & & & & & & & & & & 5.22 & 0.5 & 0.000 \\
\hline$S_{50}$ & 5.46 & 144.7 & 0.002 & & & & & & & & & & 5.22 & 0.3 & 0.002 \\
\hline
\end{tabular}


Table S17: Impact of QM cluster size on excitation energy $(\Delta E[\mathrm{eV}])$, two-photon absorption cross-section $\left(\sigma^{T P A}[\mathrm{GM}]\right)$ and one-photon oscillator strength $(f)$ for Citrine. For composition of clusters, see Tables S7-S9. Number of atoms is given in parentheses. The MM subsystem is described by electrostatic embedding.

\begin{tabular}{|c|c|c|c|c|c|c|c|c|c|c|c|c|c|c|c|c|c|c|c|c|c|}
\hline \multirow[b]{2}{*}{ State } & \multicolumn{3}{|c|}{$0.00(35)$} & \multicolumn{3}{|c|}{ int1 (51) } & \multicolumn{3}{|c|}{ int2 (65) } & \multicolumn{3}{|c|}{ int3 (77) } & \multicolumn{3}{|c|}{$0.20(87)$} & \multicolumn{3}{|c|}{$0.25(126)$} & \multicolumn{3}{|c|}{ int4 (127) } \\
\hline & $\Delta E$ & $\sigma^{T P A}$ & $f$ & $\Delta E$ & $\sigma^{T P A}$ & $f$ & $\Delta E$ & $\sigma^{T P A}$ & $f$ & $\Delta E$ & $\sigma^{T P A}$ & $f$ & $\Delta E$ & $\sigma^{T P A}$ & $f$ & $\Delta E$ & $\sigma^{T P A}$ & $f$ & $\Delta E$ & $\sigma^{T P A}$ & $f$ \\
\hline$S_{1}$ & 3.13 & 16.9 & 0.990 & 3.07 & 13.8 & 0.786 & 3.12 & 16.3 & 0.973 & 3.11 & 18.4 & 0.996 & 3.12 & 27.3 & 1.133 & 3.08 & 24.8 & 1.056 & 3.06 & 23.3 & 0.901 \\
\hline$S_{2}$ & 4.12 & 1.6 & 0.015 & 3.77 & 0.8 & 0.077 & 4.13 & 1.9 & 0.014 & 4.12 & 1.6 & 0.016 & 4.13 & 6.0 & 0.012 & 4.12 & 5.8 & 0.019 & 3.64 & 0.9 & 0.105 \\
\hline$S_{3}$ & 4.33 & 0.6 & 0.000 & 4.13 & 1.1 & 0.008 & 4.33 & 0.7 & 0.000 & 4.36 & 1.6 & 0.000 & 4.17 & 0.2 & 0.000 & 4.18 & 0.1 & 0.000 & 4.14 & 0.1 & 0.001 \\
\hline$S_{4}$ & 4.55 & 36.3 & 0.064 & 4.35 & 0.3 & 0.001 & 4.50 & 1.5 & 0.002 & 4.51 & 1.2 & 0.001 & 4.25 & 29.9 & 0.007 & 4.28 & 36.6 & 0.004 & 4.14 & 2.2 & 0.011 \\
\hline$S_{5}$ & 4.56 & 7.5 & 0.004 & 4.54 & 34.9 & 0.065 & 4.54 & 45.0 & 0.061 & 4.55 & 58.9 & 0.046 & 4.54 & 23.9 & 0.000 & 4.56 & 53.7 & 0.005 & 4.53 & 93.1 & 0.014 \\
\hline$S_{6}$ & 4.71 & 11.6 & 0.084 & 4.55 & 2.5 & 0.001 & 4.71 & 12.5 & 0.088 & 4.72 & 10.8 & 0.103 & 4.58 & 62.2 & 0.025 & 4.60 & 27.4 & 0.030 & 4.59 & 0.4 & 0.008 \\
\hline$S_{7}$ & 4.88 & 10.8 & 0.010 & 4.69 & 10.1 & 0.033 & 4.90 & 10.1 & 0.008 & 4.96 & 46.8 & 0.022 & 4.68 & 2.3 & 0.002 & 4.78 & 16.6 & 0.095 & 4.60 & 6.3 & 0.007 \\
\hline$S_{8}$ & 4.96 & 84.9 & 0.024 & 4.73 & 0.8 & 0.003 & 4.96 & 75.2 & 0.026 & 5.01 & 42.2 & 0.012 & 4.78 & 9.4 & 0.125 & 4.83 & 1.5 & 0.002 & 4.69 & 5.2 & 0.002 \\
\hline$S_{9}$ & 5.12 & 0.2 & 0.002 & 4.83 & 8.2 & 0.014 & 5.12 & 0.2 & 0.001 & 5.05 & 0.6 & 0.001 & 4.99 & 20.5 & 0.011 & 4.90 & 43.9 & 0.021 & 4.77 & 9.3 & 0.052 \\
\hline$S_{10}$ & 5.23 & 3.3 & 0.023 & 4.86 & 0.1 & 0.038 & 5.17 & 1.8 & 0.013 & 5.21 & 1.0 & 0.016 & 5.05 & 2.2 & 0.002 & 4.96 & 5.5 & 0.045 & 4.84 & 0.4 & 0.001 \\
\hline$S_{11}$ & 5.28 & 28.3 & 0.019 & 4.94 & 84.4 & 0.017 & 5.24 & 17.7 & 0.024 & 5.25 & 20.2 & 0.019 & 5.09 & 4.7 & 0.036 & 4.99 & 0.1 & 0.006 & 4.88 & 0.8 & 0.057 \\
\hline$S_{12}$ & 5.35 & 5.2 & 0.007 & 5.11 & 0.2 & 0.001 & 5.34 & 6.4 & 0.011 & 5.37 & 11.7 & 0.014 & 5.12 & 122.0 & 0.043 & 5.06 & 0.9 & 0.039 & 5.00 & 1.4 & 0.017 \\
\hline$S_{13}$ & 5.51 & 6.3 & 0.002 & 5.14 & 2.0 & 0.011 & 5.44 & 4.5 & 0.002 & 5.47 & 4.2 & 0.001 & 5.24 & 0.5 & 0.001 & 5.06 & 2.2 & 0.001 & 5.00 & 4.1 & 0.025 \\
\hline$S_{14}$ & 5.62 & 9.0 & 0.056 & 5.23 & 3.0 & 0.011 & 5.50 & 8.1 & 0.006 & 5.56 & 11.6 & 0.014 & 5.36 & 10.4 & 0.016 & 5.13 & 96.6 & 0.028 & 5.03 & 50.2 & 0.016 \\
\hline$S_{15}$ & 5.66 & 16.4 & 0.001 & 5.26 & 27.3 & 0.023 & 5.55 & 0.5 & 0.001 & 5.59 & 24.6 & 0.020 & 5.39 & 5.4 & 0.009 & 5.19 & 1.0 & 0.003 & 5.05 & 0.3 & 0.039 \\
\hline$S_{16}$ & & & & & & & & & & & & & & & & & & & 5.12 & 92.2 & 0.019 \\
\hline$S_{17}$ & & & & & & & & & & & & & & & & & & & 5.27 & 4.2 & 0.005 \\
\hline$S_{18}$ & & & & & & & & & & & & & & & & & & & 5.31 & 3.2 & 0.001 \\
\hline$S_{19}$ & & & & & & & & & & & & & & & & & & & 5.35 & 4.9 & 0.022 \\
\hline$S_{20}$ & & & & & & & & & & & & & & & & & & & 5.37 & 3.2 & 0.008 \\
\hline & \multicolumn{3}{|c|}{0.35 -noh (145) } & \multicolumn{3}{|c|}{ int5 (166) } & \multicolumn{3}{|c|}{$0.30(167)$} & \multicolumn{3}{|c|}{ int6 (196) } & \multicolumn{3}{|c|}{ int7 (202) } & \multicolumn{3}{|c|}{$0.35(208)$} & \multicolumn{3}{|c|}{$\overline{\text { int8 (217) }}$} \\
\hline$S_{1}$ & 3.04 & 20.7 & 0.842 & 3.01 & 20.3 & 0.836 & 3.04 & 23.2 & 1.001 & 2.98 & 18.1 & 0.785 & 2.98 & 20.1 & 0.819 & 2.98 & 16.1 & 0.746 & 2.98 & 19.3 & 0.811 \\
\hline$S_{2}$ & 3.65 & 0.9 & 0.091 & 3.61 & 0.9 & 0.101 & 4.08 & 3.2 & 0.019 & 3.62 & 1.0 & 0.088 & 3.60 & 1.0 & 0.097 & 3.63 & 0.9 & 0.082 & 3.63 & 1.0 & 0.090 \\
\hline$S_{3}$ & 4.16 & 2.2 & 0.011 & 4.13 & 12.1 & 0.008 & 4.09 & 1.4 & 0.008 & 4.09 & 4.6 & 0.016 & 4.10 & 7.6 & 0.016 & 4.09 & 4.3 & 0.015 & 4.09 & 5.3 & 0.016 \\
\hline$S_{4}$ & 4.21 & 0.1 & 0.000 & 4.19 & 17.2 & 0.007 & 4.34 & 44.4 & 0.002 & 4.14 & 0.1 & 0.000 & 4.15 & 0.2 & 0.000 & 4.15 & 0.1 & 0.000 & 4.15 & 0.1 & 0.000 \\
\hline$S_{5}$ & 4.53 & 94.7 & 0.020 & 4.20 & 0.1 & 0.000 & 4.55 & 14.9 & 0.001 & 4.27 & 30.0 & 0.003 & 4.21 & 22.9 & 0.005 & 4.27 & 27.3 & 0.002 & 4.25 & 28.8 & 0.003 \\
\hline$S_{6}$ & 4.61 & 1.5 & 0.014 & 4.55 & 82.9 & 0.018 & 4.60 & 54.1 & 0.048 & 4.56 & 51.7 & 0.010 & 4.55 & 78.8 & 0.023 & 4.56 & 52.7 & 0.015 & 4.56 & 63.9 & 0.015 \\
\hline$S_{7}$ & 4.62 & 1.6 & 0.004 & 4.57 & 3.3 & 0.002 & 4.73 & 0.6 & 0.001 & 4.57 & 0.8 & 0.004 & 4.55 & 0.5 & 0.003 & 4.58 & 0.6 & 0.004 & 4.58 & 0.7 & 0.005 \\
\hline$S_{8}$ & 4.75 & 10.7 & 0.045 & 4.61 & 1.3 & 0.016 & 4.76 & 17.7 & 0.076 & 4.58 & 18.7 & 0.034 & 4.59 & 3.1 & 0.022 & 4.59 & 11.7 & 0.029 & 4.59 & 13.3 & 0.030 \\
\hline$S_{9}$ & 4.86 & 1.9 & 0.034 & 4.75 & 12.8 & 0.043 & 4.83 & 46.1 & 0.023 & 4.70 & 0.3 & 0.002 & 4.73 & 13.2 & 0.031 & 4.69 & 0.3 & 0.002 & 4.70 & 0.4 & 0.002 \\
\hline$S_{10}$ & 4.88 & 12.9 & 0.030 & 4.85 & 10.1 & 0.001 & 4.89 & 0.0 & 0.002 & 4.73 & 14.2 & 0.035 & 4.75 & 16.8 & 0.013 & 4.74 & 14.9 & 0.034 & 4.73 & 14.8 & 0.035 \\
\hline$S_{11}$ & 4.91 & 0.1 & 0.001 & 4.87 & 1.7 & 0.056 & 4.90 & 7.5 & 0.045 & 4.82 & 37.9 & 0.017 & 4.83 & 19.7 & 0.010 & 4.83 & 36.0 & 0.016 & 4.81 & 41.7 & 0.017 \\
\hline$S_{12}$ & 4.98 & 36.3 & 0.007 & 4.91 & 4.0 & 0.004 & 5.04 & 1.1 & 0.044 & 4.86 & 0.3 & 0.042 & 4.86 & 0.3 & 0.036 & 4.86 & 0.4 & 0.038 & 4.86 & 0.3 & 0.041 \\
\hline$S_{13}$ & 4.99 & 4.5 & 0.042 & 4.91 & 24.7 & 0.007 & 5.07 & 91.9 & 0.024 & 4.88 & 0.0 & 0.001 & 4.88 & 6.9 & 0.056 & 4.88 & 6.7 & 0.053 & 4.88 & 6.6 & 0.053 \\
\hline$S_{14}$ & 5.00 & 2.7 & 0.001 & 4.98 & 4.6 & 0.041 & 5.08 & 9.9 & 0.002 & 4.89 & 6.7 & 0.051 & 4.96 & 0.1 & 0.004 & 4.89 & 0.3 & 0.000 & 4.89 & 0.2 & 0.000 \\
\hline$S_{15}$ & 5.05 & 0.6 & 0.040 & 5.04 & 1.8 & 0.001 & 5.12 & 0.1 & 0.000 & 5.03 & 2.8 & 0.044 & 5.04 & 91.4 & 0.021 & 5.03 & 0.8 & 0.041 & 5.02 & 64.6 & 0.015 \\
\hline$S_{16}$ & & & & & & & & & & 5.04 & 69.4 & 0.010 & & & & & & & & & \\
\hline$S_{17}$ & & & & & & & & & & 5.07 & 2.5 & 0.001 & & & & & & & & & \\
\hline$S_{18}$ & & & & & & & & & & 5.10 & 0.1 & 0.004 & & & & & & & & & \\
\hline$S_{19}$ & & & & & & & & & & 5.13 & 1.9 & 0.002 & & & & & & & & & \\
\hline$S_{20}$ & & & & & & & & & & 5.15 & 0.7 & 0.002 & & & & & & & & & \\
\hline & & $0.40(24$ & & & int9 (24 & & & $0.45(30$ & & & $0.50(3$ & & & & & & & & & & \\
\hline
\end{tabular}




\begin{tabular}{l|ccc|ccc|ccc|ccc|l}
\hline$S_{1}$ & 2.98 & 17.4 & 0.773 & 2.98 & 17.5 & 0.774 & 3.00 & 18.2 & 0.787 & 3.00 & 17.2 & 0.762 & \\
$S_{2}$ & 3.64 & 0.8 & 0.084 & 3.64 & 0.9 & 0.084 & 3.68 & 0.8 & 0.078 & 3.68 & 0.7 & 0.078 & \\
$S_{3}$ & 4.10 & 5.1 & 0.015 & 4.10 & 4.7 & 0.015 & 4.13 & 6.4 & 0.011 & 4.13 & 6.0 & 0.011 & \\
$S_{4}$ & 4.20 & 0.0 & 0.000 & 4.18 & 0.0 & 0.000 & 4.27 & 26.6 & 0.003 & 4.28 & 27.4 & 0.003 & \\
$S_{5}$ & 4.26 & 26.5 & 0.003 & 4.27 & 27.7 & 0.003 & 4.28 & 0.1 & 0.000 & 4.37 & 0.0 & 0.000 & \\
$S_{6}$ & 4.56 & 68.8 & 0.027 & 4.56 & 66.3 & 0.024 & 4.55 & 73.4 & 0.031 & 4.56 & 69.7 & 0.032 & \\
$S_{7}$ & 4.59 & 0.8 & 0.004 & 4.59 & 0.7 & 0.006 & 4.62 & 2.5 & 0.020 & 4.63 & 1.9 & 0.003 & \\
$S_{8}$ & 4.60 & 2.3 & 0.019 & 4.60 & 4.6 & 0.021 & 4.64 & 1.0 & 0.000 & 4.64 & 2.8 & 0.021 & \\
$S_{9}$ & 4.74 & 13.0 & 0.036 & 4.70 & 0.5 & 0.002 & 4.75 & 12.2 & 0.032 & & & & \\
$S_{10}$ & 4.77 & 1.7 & 0.001 & 4.74 & 14.9 & 0.034 & 4.78 & 1.6 & 0.002 & & & & \\
$S_{11}$ & 4.84 & 33.4 & 0.016 & 4.83 & 36.1 & 0.015 & 4.84 & 6.0 & 0.024 & & & & \\
$S_{12}$ & 4.86 & 0.5 & 0.032 & 4.86 & 0.4 & 0.035 & 4.85 & 30.3 & 0.028 & & & & \\
$S_{13}$ & 4.87 & 6.9 & 0.058 & 4.88 & 6.9 & 0.057 & 4.86 & 1.8 & 0.052 & & & & \\
$S_{14}$ & 4.93 & 0.1 & 0.002 & 4.91 & 0.1 & 0.001 & 5.00 & 0.3 & 0.020 & & & & \\
$S_{15}$ & 5.03 & 0.9 & 0.040 & 5.03 & 1.1 & 0.041 & 5.00 & 2.8 & 0.002 & & & & \\
\hline \hline
\end{tabular}

Table S18: Impact of QM cluster size on excitation energy $(\Delta E[\mathrm{eV}])$, two-photon absorption cross-section $\left(\sigma^{T P A}[\mathrm{GM}]\right)$ and one-photon oscillator strength $(f)$ for Citrine. For composition of clusters, see Tables S7-S9. Number of atoms is given in parentheses. The MM subsystem is described by polarizable embedding.

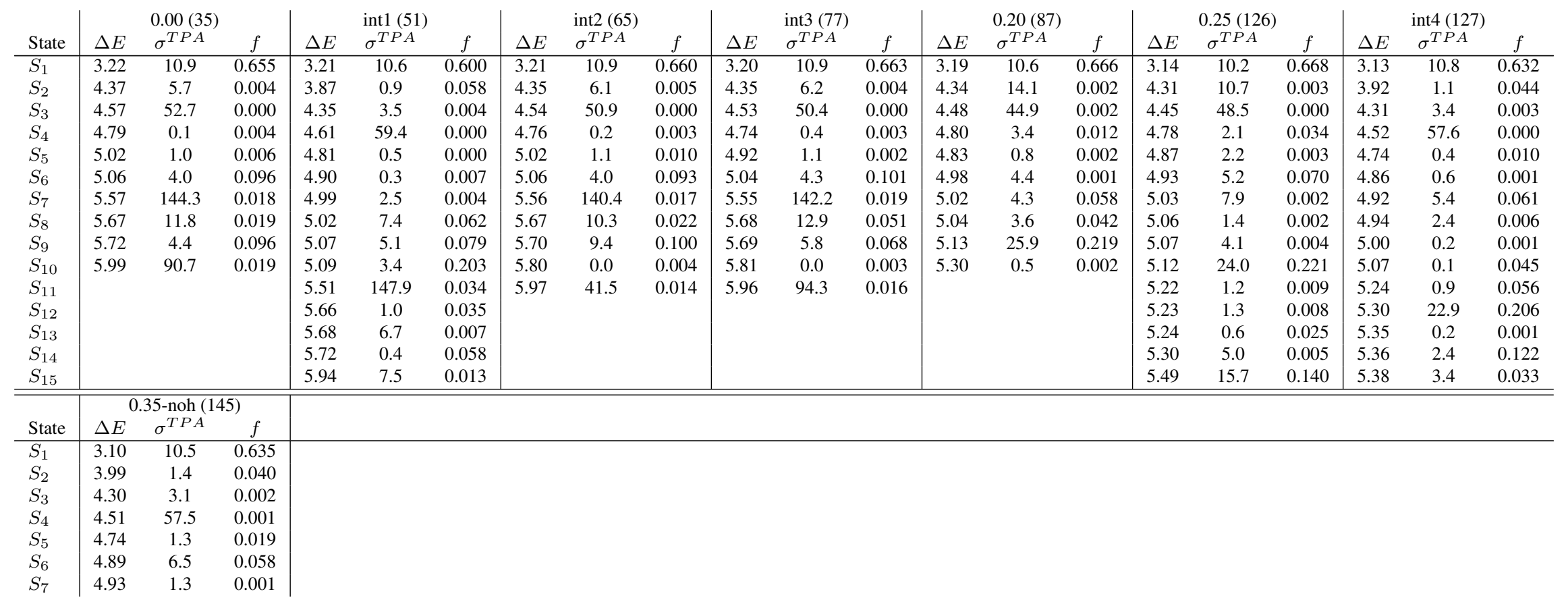




\begin{tabular}{l|ccc|l|}
$S_{8}$ & 4.93 & 0.9 & 0.002 & \\
$S_{9}$ & 5.05 & 0.2 & 0.002 & \\
$S_{10}$ & 5.06 & 0.1 & 0.044 & \\
$S_{11}$ & 5.23 & 1.1 & 0.066 & \\
$S_{12}$ & 5.27 & 21.7 & 0.123 & \\
$S_{13}$ & 5.29 & 7.0 & 0.074 & \\
$S_{14}$ & 5.34 & 1.6 & 0.108 & \\
$S_{15}$ & 5.35 & 0.2 & 0.001 & \\
\hline \hline
\end{tabular}




\section{S6 Remaining supporting information}

S6.1 Comparison of $\triangle E, \sigma^{T P A}$ and $f$ values obtained with BHandHLYP and CAM-B3LYP functionals for chosen models of avGFP-n and Citrine

Table S19: Impact of QM cluster size on excitation energy ( $\Delta E[\mathrm{eV}])$, two-photon absorption cross-section $\left(\sigma^{T P A}[\mathrm{GM}]\right)$ and one-photon oscillator strength $(f)$ for avGFP-n. For composition of clusters, see Tables S1-S3. Number of atoms is given in parentheses. The MM subsystem is described by electrostatic embedding.

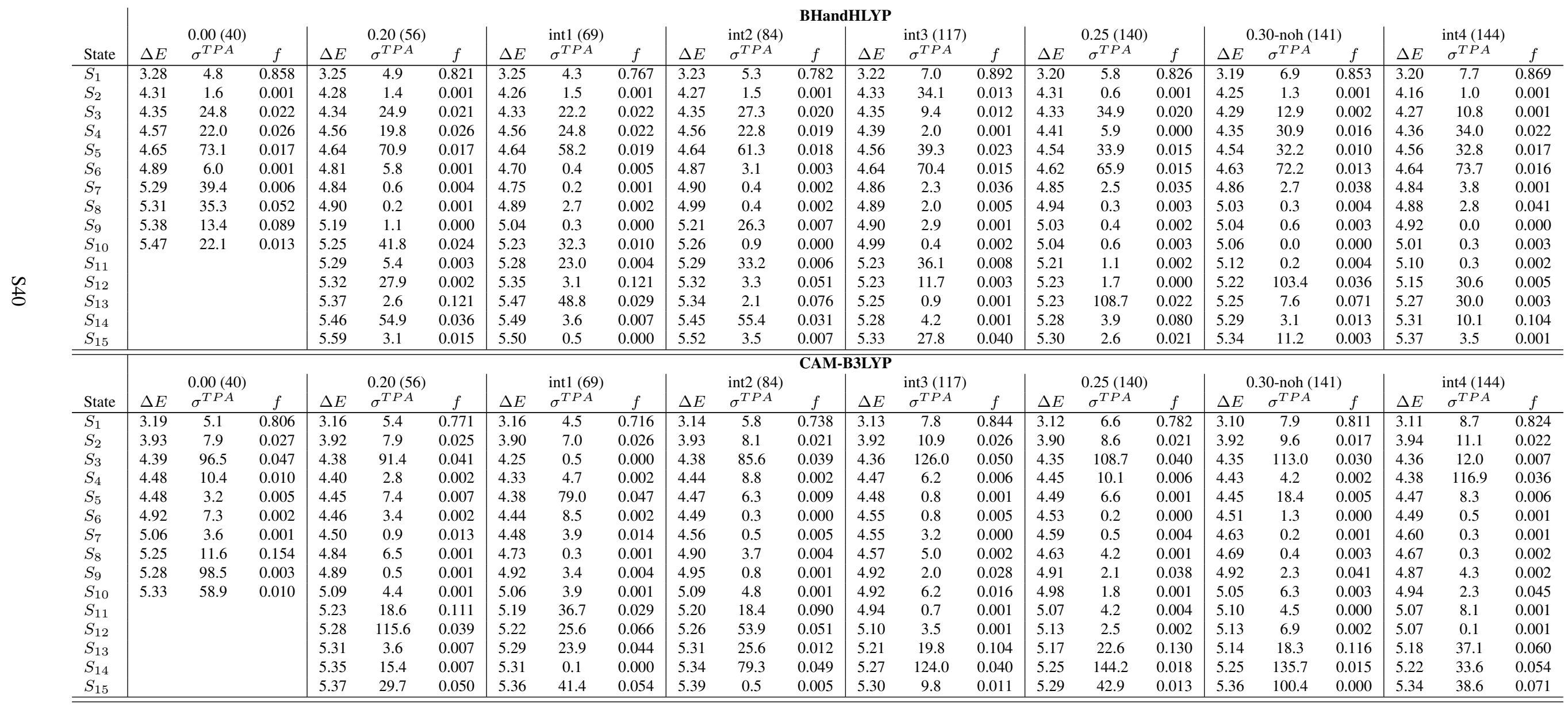


Table S20: Impact of QM cluster size on excitation energy ( $\triangle E[\mathrm{eV}])$, two-photon absorption cross-section $\left(\sigma^{T P A}[\mathrm{GM}]\right)$ and one-photon oscillator strength $(f)$ for Citrine. For composition of clusters, see Tables S7-S9. Number of atoms is given in parentheses. The MM subsystem is described by electrostatic embedding.

\begin{tabular}{|c|c|c|c|c|c|c|c|c|c|c|c|c|c|c|c|c|c|c|c|c|c|c|c|c|}
\hline & & & & & & & & & & & & BHan & HLYP & & & & & & & & & & & \\
\hline & & $0.00(35)$ & & & int1 (51) & & & int2 (65) & & & int3 (77) & & & $0.20(87$ & & & $0.25(12 \theta$ & & & int4 (127 & & & $35-\operatorname{noh}(1$ & \\
\hline State & $\Delta E$ & $\sigma^{T P}$ & $f$ & $\Delta E$ & $\sigma^{T P}$ & $f$ & $\Delta E$ & $\sigma^{T P}$ & $f$ & $\Delta E$ & $\sigma^{T P A}$ & $f$ & $\Delta E$ & $\sigma^{T P A}$ & $f$ & $\Delta E$ & $\sigma^{T P A}$ & $f$ & $\Delta E$ & $\sigma^{T P A}$ & $f$ & $\Delta E$ & $\sigma^{T P A}$ & $f$ \\
\hline$S_{1}$ & 3.13 & 16.9 & 0.990 & 3.07 & 13.8 & 0.786 & 3.12 & 16.3 & 0.973 & 3.11 & 18.4 & 0.996 & 3.12 & 27.3 & 1.133 & 3.08 & 24.8 & 1.056 & 3.06 & 23.3 & 0.901 & 3.04 & 20.7 & 0.842 \\
\hline$S_{2}$ & 4.12 & 1.6 & 0.015 & 3.77 & 0.8 & 0.077 & 4.13 & 1.9 & 0.014 & 4.12 & 1.6 & 0.016 & 4.13 & 6.0 & 0.012 & 4.12 & 5.8 & 0.019 & 3.64 & 0.9 & 0.105 & 3.65 & 0.9 & 0.091 \\
\hline$S_{3}$ & 4.33 & 0.6 & 0.000 & 4.13 & 1.1 & 0.008 & 4.33 & 0.7 & 0.000 & 4.36 & 1.6 & 0.000 & 4.17 & 0.2 & 0.000 & 4.18 & 0.1 & 0.000 & 4.14 & 0.1 & 0.001 & 4.16 & 2.2 & 0.011 \\
\hline$S_{4}$ & 4.55 & 36.3 & 0.064 & 4.35 & 0.3 & 0.001 & 4.50 & 1.5 & 0.002 & 4.51 & 1.2 & 0.001 & 4.25 & 29.9 & 0.007 & 4.28 & 36.6 & 0.004 & 4.14 & 2.2 & 0.011 & 4.21 & 0.1 & 0.000 \\
\hline$S_{5}$ & 4.56 & 7.5 & 0.004 & 4.54 & 34.9 & 0.065 & 4.54 & 45.0 & 0.061 & 4.55 & 58.9 & 0.046 & 4.54 & 23.9 & 0.000 & 4.56 & 53.7 & 0.005 & 4.53 & 93.1 & 0.014 & 4.53 & 94.7 & 0.020 \\
\hline$S_{6}$ & 4.71 & 11.6 & 0.084 & 4.55 & 2.5 & 0.001 & 4.71 & 12.5 & 0.088 & 4.72 & 10.8 & 0.103 & 4.58 & 62.2 & 0.025 & 4.60 & 27.4 & 0.030 & 4.59 & 0.4 & 0.008 & 4.61 & 1.5 & 0.014 \\
\hline$S_{7}$ & 4.88 & 10.8 & 0.010 & 4.69 & 10.1 & 0.033 & 4.90 & 10.1 & 0.008 & 4.96 & 46.8 & 0.022 & 4.68 & 2.3 & 0.002 & 4.78 & 16.6 & 0.095 & 4.60 & 6.3 & 0.007 & 4.62 & 1.6 & 0.004 \\
\hline$S_{8}$ & 4.96 & 84.9 & 0.024 & 4.73 & 0.8 & 0.003 & 4.96 & 75.2 & 0.026 & 5.01 & 42.2 & 0.012 & 4.78 & 9.4 & 0.125 & 4.83 & 1.5 & 0.002 & 4.69 & 5.2 & 0.002 & 4.75 & 10.7 & 0.045 \\
\hline$S_{9}$ & 5.12 & 0.2 & 0.002 & 4.83 & 8.2 & 0.014 & 5.12 & 0.2 & 0.001 & 5.05 & 0.6 & 0.001 & 4.99 & 20.5 & 0.011 & 4.90 & 43.9 & 0.021 & 4.77 & 9.3 & 0.052 & 4.86 & 1.9 & 0.034 \\
\hline$S_{10}$ & 5.23 & 3.3 & 0.023 & 4.86 & 0.1 & 0.038 & 5.17 & 1.8 & 0.013 & 5.21 & 1.0 & 0.016 & 5.05 & 2.2 & 0.002 & 4.96 & 5.5 & 0.045 & 4.84 & 0.4 & 0.001 & 4.88 & 12.9 & 0.030 \\
\hline$S_{11}$ & 5.28 & 28.3 & 0.019 & 4.94 & 84.4 & 0.017 & 5.24 & 17.7 & 0.024 & 5.25 & 20.2 & 0.019 & 5.09 & 4.7 & 0.036 & 4.99 & 0.1 & 0.006 & 4.88 & 0.8 & 0.057 & 4.91 & 0.1 & 0.001 \\
\hline$S_{12}$ & 5.35 & 5.2 & 0.007 & 5.11 & 0.2 & 0.001 & 5.34 & 6.4 & 0.011 & 5.37 & 11.7 & 0.014 & 5.12 & 122.0 & 0.043 & 5.06 & 0.9 & 0.039 & 5.00 & 1.4 & 0.017 & 4.98 & 36.3 & 0.007 \\
\hline$S_{13}$ & 5.51 & 6.3 & 0.002 & 5.14 & 2.0 & 0.011 & 5.44 & 4.5 & 0.002 & 5.47 & 4.2 & 0.001 & 5.24 & 0.5 & 0.001 & 5.06 & 2.2 & 0.001 & 5.00 & 4.1 & 0.025 & 4.99 & 4.5 & 0.042 \\
\hline$S_{14}$ & 5.62 & 9.0 & 0.056 & 5.23 & 3.0 & 0.011 & 5.50 & 8.1 & 0.006 & 5.56 & 11.6 & 0.014 & 5.36 & 10.4 & 0.016 & 5.13 & 96.6 & 0.028 & 5.03 & 50.2 & 0.016 & 5.00 & 2.7 & 0.001 \\
\hline$S_{15}$ & 5.66 & 16.4 & 0.001 & 5.26 & 27.3 & 0.023 & 5.55 & 0.5 & 0.001 & 5.59 & 24.6 & 0.020 & 5.39 & 5.4 & 0.009 & 5.19 & 1.0 & 0.003 & 5.05 & 0.3 & 0.039 & 5.05 & 0.6 & 0.040 \\
\hline & & & & & & & & & & & & 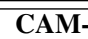 & $\overline{3 \mathrm{LLYP}}$ & & & & & & & & & & & \\
\hline & & $0.00(35)$ & & & int1 (51) & & & int2 (65) & & & int3 (77) & & & $0.20(87$ & & & 0.25 & & & int4 (1 & & & 35-noh (1 & \\
\hline State & $\Delta E$ & $\sigma^{T P A}$ & $f$ & $\Delta E$ & $\sigma^{T P A}$ & $f$ & $\Delta E$ & $\sigma^{T P A}$ & $f$ & $\Delta E$ & $\sigma^{T P A}$ & $f$ & $\Delta E$ & $\sigma^{T P A}$ & $f$ & $\Delta E$ & $\sigma^{T P A}$ & $f$ & $\Delta E$ & $\sigma^{T P A}$ & $f$ & $\Delta E$ & $\sigma^{T P A}$ & $f$ \\
\hline$S_{1}$ & 3.03 & 16.3 & 0.961 & 2.97 & 13.4 & 0.773 & 3.02 & 15.7 & 0.944 & 3.01 & 18.0 & 0.968 & 3.01 & 27.4 & 1.104 & 2.97 & 25.2 & 1.027 & 2.96 & 23.9 & 0.892 & 2.93 & 21.3 & 0.829 \\
\hline$S_{2}$ & 3.89 & 0.3 & 0.008 & 3.72 & 1.1 & 0.063 & 3.89 & 0.3 & 0.007 & 3.92 & 0.2 & 0.008 & 4.01 & 5.3 & 0.008 & 4.01 & 6.1 & 0.012 & 3.58 & 1.0 & 0.085 & 3.59 & 1.0 & 0.076 \\
\hline$S_{3}$ & 4.12 & 2.9 & 0.003 & 3.92 & 0.2 & 0.004 & 4.12 & 3.5 & 0.002 & 4.11 & 4.3 & 0.004 & 4.12 & 44.9 & 0.007 & 4.15 & 52.9 & 0.005 & 4.03 & 2.0 & 0.007 & 4.04 & 2.3 & 0.007 \\
\hline$S_{4}$ & 4.37 & 36.2 & 0.033 & 4.12 & 1.9 & 0.001 & 4.36 & 39.7 & 0.032 & 4.36 & 50.6 & 0.020 & 4.21 & 1.2 & 0.000 & 4.24 & 0.9 & 0.000 & 4.24 & 16.1 & 0.001 & 4.26 & 20.5 & 0.000 \\
\hline$S_{5}$ & 4.55 & 4.8 & 0.071 & 4.36 & 32.3 & 0.033 & 4.50 & 1.6 & 0.005 & 4.52 & 1.7 & 0.005 & 4.36 & 0.4 & 0.000 & 4.36 & 0.3 & 0.000 & 4.32 & 2.0 & 0.000 & 4.34 & 63.2 & 0.012 \\
\hline$S_{6}$ & 4.58 & 5.4 & 0.038 & 4.54 & 5.2 & 0.059 & 4.56 & 7.7 & 0.106 & 4.57 & 6.2 & 0.113 & 4.39 & 57.8 & 0.009 & 4.40 & 51.9 & 0.015 & 4.35 & 68.9 & 0.010 & 4.39 & 0.1 & 0.001 \\
\hline$S_{7}$ & 4.72 & 0.4 & 0.006 & 4.56 & 4.1 & 0.001 & 4.72 & 0.3 & 0.004 & 4.65 & 1.5 & 0.009 & 4.62 & 4.7 & 0.112 & 4.62 & 8.8 & 0.101 & 4.58 & 1.1 & 0.000 & 4.59 & 2.1 & 0.028 \\
\hline$S_{8}$ & 4.95 & 116.2 & 0.043 & 4.69 & 0.4 & 0.028 & 4.96 & 118.6 & 0.049 & 4.98 & 130.4 & 0.050 & 4.66 & 4.4 & 0.029 & 4.67 & 6.9 & 0.019 & 4.58 & 1.0 & 0.012 & 4.60 & 1.4 & 0.003 \\
\hline$S_{9}$ & 5.04 & 26.4 & 0.007 & 4.70 & 0.4 & 0.003 & 5.06 & 8.3 & 0.005 & 5.12 & 4.5 & 0.008 & 4.69 & 2.6 & 0.002 & 4.89 & 0.8 & 0.042 & 4.62 & 8.9 & 0.057 & 4.61 & 8.2 & 0.040 \\
\hline$S_{10}$ & 5.21 & 9.5 & 0.015 & 4.76 & 0.2 & 0.015 & 5.16 & 3.5 & 0.013 & 5.21 & 3.5 & 0.009 & 5.05 & 151.3 & 0.050 & 4.95 & 61.5 & 0.021 & 4.71 & 2.9 & 0.003 & 4.72 & 0.3 & 0.053 \\
\hline$S_{11}$ & 5.29 & 5.8 & 0.010 & 4.93 & 89.2 & 0.032 & 5.27 & 7.7 & 0.010 & 5.29 & 8.6 & 0.009 & 5.10 & 23.3 & 0.011 & 4.99 & 81.8 & 0.020 & 4.73 & 0.4 & 0.053 & 4.89 & 0.7 & 0.038 \\
\hline$S_{12}$ & 5.35 & 4.0 & 0.003 & 5.01 & 54.7 & 0.008 & 5.34 & 8.3 & 0.004 & 5.35 & 10.8 & 0.004 & 5.12 & 4.6 & 0.044 & 5.00 & 5.2 & 0.048 & 4.88 & 0.4 & 0.025 & 4.98 & 72.9 & 0.015 \\
\hline$S_{13}$ & 5.36 & 10.8 & 0.038 & 5.15 & 4.9 & 0.013 & 5.35 & 10.9 & 0.032 & 5.37 & 48.0 & 0.042 & 5.20 & 21.7 & 0.005 & 5.07 & 0.1 & 0.001 & 4.92 & 0.3 & 0.015 & 4.98 & 1.3 & 0.004 \\
\hline$S_{14}$ & 5.45 & 280.1 & 0.009 & 5.24 & 4.7 & 0.008 & 5.44 & 220.1 & 0.003 & 5.46 & 617.1 & 0.015 & 5.35 & 20.4 & 0.007 & 5.10 & 27.1 & 0.009 & 5.04 & 4.1 & 0.039 & 5.03 & 3.9 & 0.041 \\
\hline$S_{15}$ & 5.49 & 240.9 & 0.043 & 5.27 & 3.7 & 0.030 & 5.48 & 307.0 & 0.040 & 5.50 & 36.0 & 0.019 & 5.36 & 6.8 & 0.008 & 5.18 & 36.9 & 0.009 & 5.06 & 198.2 & 0.044 & 5.05 & 112.1 & 0.019 \\
\hline
\end{tabular}



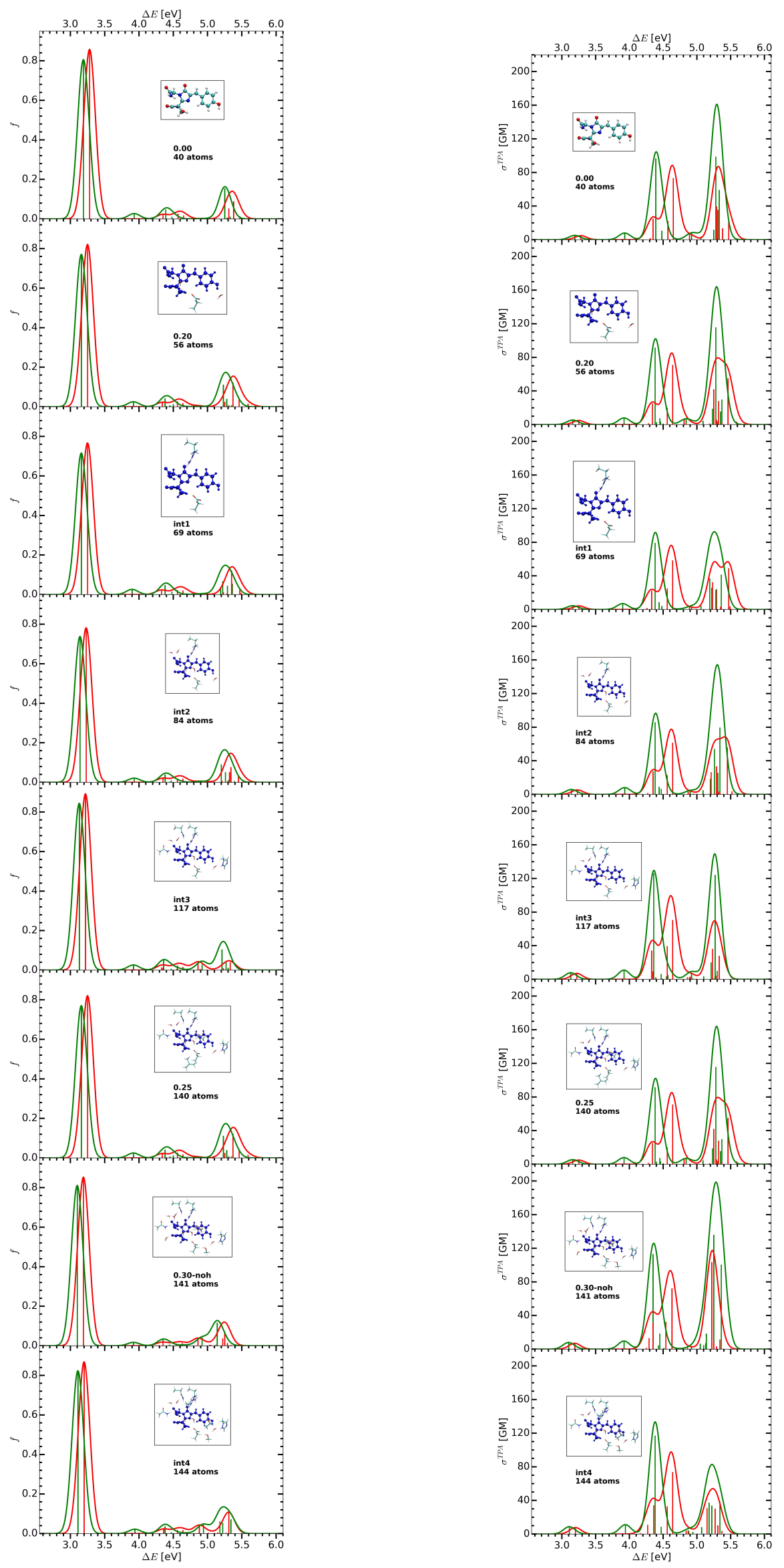

Figure S12: OPA (left) and TPA (right) spectra for chosen QM subsystems of avGFP-n models obtained at BHandHLYP/EE (red) and CAM-B3LYP/EE (green) levels of theory. The values of $\triangle E, \sigma^{T P A}$ and $f$ are availabe in Table S19. 

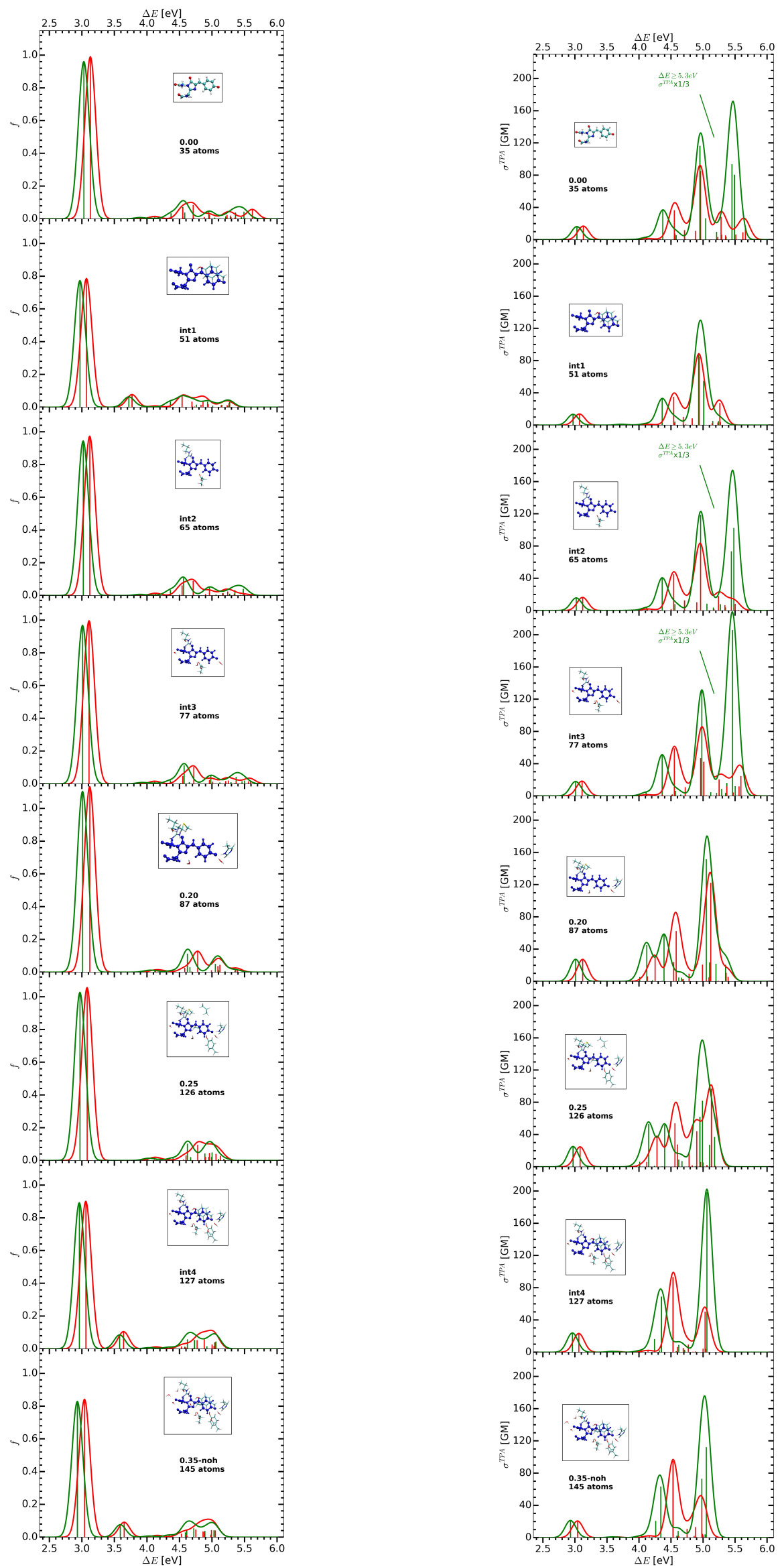

Figure S13: OPA (left) and TPA (right) spectra for chosen QM subsystems of Citrine models obtained at BHandHLYP/EE (red) and CAM-B3LYP/EE (green) levels of theory. The values of $\Delta E, \sigma^{T P A}$ and $f$ are availabe in Table S20. 
According to OPA and TPA spectra in Figures S12-S13, the CAM-B3LYP functional usually predicts red-shift of ca. $0.1-0.2 \mathrm{eV}$ in absorption peaks position compared to BHandHLYP functional. Nevertheless, in case of the $S_{0} \rightarrow S_{1}$ transitions, we observe quantitatively the same trends in $\Delta E, \sigma^{T P A}$ and $f$ values as a function of the QM region size using either of the functionals (Tables S19-S20). It is somehow less evident for excitations to higher excited states because according to BHandHLYP functional, the brightest TPA bands are more more fragmented in terms of excited states number. However, the effect of extending the QM region size is similar when the Gaussian-convoluted TPA bands are analyzed (Figures $\mathrm{S} 12-\mathrm{S} 13$ ) at least for $\Delta E$ up to ca. $5.2 \mathrm{eV}$. For higher-lying excited states, there are more visible differences in $\sigma^{T P A}$ values obtained with BHandHLYP and CAM-B3LYP functionals. This is presumably due to plausible charge-transfer transitions in this excitation energies range. Contrary to BHandHLYP functional, CAM-B3LYP functional is better suited to describe spectral properties related to such excitations [5].

\section{S6.2 Comparison of $\triangle E, \sigma^{T P A}$ and $f$ values obtained in PE approximation with water molecules included in 3.0, 5.0, and 7.0 $\AA$ radius from protein}

Table S21: Excitation energy ( $\Delta E[\mathrm{eV}])$, two-photon absorption cross-section $\left(\sigma^{T P A}[\mathrm{GM}]\right)$ and one-photon oscillator strength $(f)$ for $\mathbf{0 . 0 0 / P E}$ model of avGFP-n with water molecules included in the polarizable force field within 3.0, 5.0 and 7.0 ̊ radius from the protein. Number of water molecules included in PE is given together with time required to converge linear and quadratic response functions.

\begin{tabular}{l|ccc|ccc|ccc} 
& \multicolumn{5}{|c|}{$3.0 \AA$} & \multicolumn{3}{c|}{$5.0 \AA$} & \multicolumn{3}{c}{$7.0 \AA$} \\
State & $\Delta E$ & $\sigma^{T P A}$ & $f$ & $\Delta E$ & $\sigma^{T P A}$ & $f$ & $\Delta E$ & $\sigma^{T P A}$ \\
\hline$S_{1}$ & 3.26 & 0.7 & 0.493 & 3.27 & 0.9 & 0.509 & 3.26 & 1.2 & 0.521 \\
$S_{2}$ & 4.25 & 6.9 & 0.021 & 4.28 & 6.6 & 0.017 & 4.30 & 7.7 & 0.016 \\
$S_{3}$ & 4.60 & 53.1 & 0.038 & 4.63 & 56.6 & 0.038 & 4.64 & 57.7 & 0.033 \\
$S_{4}$ & 4.64 & 1.1 & 0.031 & 4.65 & 4.0 & 0.025 & 4.67 & 5.7 & 0.024 \\
$S_{5}$ & 5.34 & 39.3 & 0.033 & 5.46 & 48.8 & 0.036 & 5.50 & 74.4 & 0.024 \\
$S_{6}$ & 5.53 & 98.8 & 0.030 & 5.52 & 107.4 & 0.046 & 5.51 & 74.9 & 0.074 \\
$S_{7}$ & 5.56 & 10.5 & 0.008 & 5.59 & 5.3 & 0.006 & 5.58 & 6.3 & 0.010 \\
$S_{8}$ & 5.61 & 4.0 & 0.006 & 5.62 & 6.3 & 0.015 & 5.62 & 16.9 & 0.019 \\
$S_{9}$ & 5.68 & 32.3 & 0.068 & 5.69 & 26.7 & 0.049 & 5.69 & 24.3 & 0.032 \\
$S_{10}$ & 5.78 & 1.7 & 0.006 & 5.79 & 1.8 & 0.005 & 5.80 & 3.0 & 0.005 \\
\hline $\mathrm{N}$ & \multicolumn{3}{|c}{618} & 1251 & \multicolumn{5}{c}{1992} \\
Time [h] & $7.5 / 24 \mathrm{CPUs}$ & \multicolumn{3}{c}{$20.3 / 12 \mathrm{CPUs}$} & $30.1 / 12 \mathrm{CPUs}$ \\
\hline \hline
\end{tabular}

Table S22: Excitation energy $(\Delta E[\mathrm{eV}])$, two-photon absorption cross-section $\left(\sigma^{T P A}[\mathrm{GM}]\right)$ and one-photon oscillator strength $(f)$ for $\mathbf{0 . 0 0 / P E}$ model of avGFP-a with water molecules included in the polarizable force field within 3.0, 5.0 and 7.0 $\AA$ radius from the protein. Number of water molecules included in PE is given together with time required to converge linear and quadratic response functions.

\begin{tabular}{l|ccc|ccc|ccc} 
& \multicolumn{3}{|c|}{$3.0 \AA$} & \multicolumn{3}{c|}{$5.0 \AA$} & \multicolumn{3}{c}{$7.0 \AA$} \\
State & $\Delta E$ & $\sigma^{T P A}$ & $f$ & $\Delta E$ & $\sigma^{T P A}$ & $f$ & $\Delta E$ & $\sigma^{T P A}$ \\
\hline$S_{1}$ & 3.10 & 3.1 & 0.691 & 3.09 & 3.2 & 0.700 & 3.09 & 3.2 & 0.709 \\
$S_{2}$ & 4.47 & 30.0 & 0.013 & 4.48 & 26.5 & 0.012 & 4.48 & 26.7 & 0.012 \\
$S_{3}$ & 4.55 & 21.3 & 0.009 & 4.56 & 26.9 & 0.009 & 4.56 & 28.2 & 0.009 \\
$S_{4}$ & 4.66 & 14.2 & 0.003 & 4.68 & 13.8 & 0.003 & 4.68 & 13.2 & 0.003 \\
$S_{5}$ & 4.94 & 0.5 & 0.007 & 4.89 & 0.4 & 0.005 & 4.87 & 0.4 & 0.005 \\
$S_{6}$ & 5.20 & 4.9 & 0.103 & 5.18 & 5.4 & 0.107 & 5.17 & 5.7 & 0.108 \\
\hline $\mathrm{N}$ & \multicolumn{4}{|c|}{585} & \multicolumn{4}{c|}{1243} & \multicolumn{3}{c}{2019} \\
Time [h] & $6.5 / 24$ CPUs & $20.0 / 12$ CPUs & $30.5 / 12$ CPUs \\
\hline \hline
\end{tabular}

The computations cost increases with a number of polarization sites, because the induced dipoles are optimized for ground and each electronic excitation until self-consistency, in terms of induced dipoles vector components values, is reached [6]. The impact of water molecules more distant than $3.0 \AA$ from the protein on the $\Delta E, \sigma^{T P A}$ and $f$ values 
of the $S_{0} \rightarrow S_{1}$ transition in avGFP models with both neutral and anionic chromophore is very small (Tables S21-S22). This is quite similarly the case for higher excited states spectral properties. In case of avGFP-n, the $S_{3}$ excited state, displaying large $\sigma^{T P A}$ value, gains by up to $4.6 \mathrm{GM}$ in TPA intensity as the cut-off radius for neglecting water molecules increases from $3.0 \AA$ to $7.0 \AA$. This is only $8.7 \%$ in reference to value obtained with the shortest cut-off radius. The excitation energy becomes blue-shifted by $0.04 \mathrm{eV}$ which is well below the accuracy of utilized TDDFT functionals [7,8]. Quite surprisingly, the effect of including more water molecules in polarizable force field is smaller for avGFP-a model (Table S22). For a bunch of $S_{2}-S_{4}$ excited states, the change in $\Delta E$ is only $0.01-0.02 \mathrm{eV}$ when results obtained with 3.0 $\AA$ vs. $5.0 \AA$ cut-off are compared, and is reduced to zero for $5.0 \AA$ vs $7.0 \AA$ cut-off. We also observe some TPA intensity transfer between $S_{2}$ and $S_{3}$ excited states with cut-off radius above $3.0 \AA$. Nevertheless, the sum of $\sigma^{T P A}$ for $S_{2}-S_{4}$ excited states is in range of 65.5-68.1 GM for three cut-off values, thus the overall effect is rather negligible. The same does not apply for TPA bright excited states $S_{5}-S_{6}$ in avGFP-n, where we observe more extensive TPA intensity transfer that leads to sum $\sigma^{T P A}$ values in range of 138.1-156.2 GM (Table S21).

Since the chromophore size along long axis is about $10 \AA$ and the chromophore is roughly placed centrally in the $\beta$-barrel that is about $24 \AA$ in diameter, we estimate that we include electrostatic and polarization interactions between the $0.00 \mathrm{QM}$ region and water molecules that are up to ca. $10 \AA$ from the chromophore plane. According to Beerepoot et al. [9] including more distant polarizable sites leads to changes in $\Delta E, \sigma^{T P A}$, and $f$ values up to ca. $0.02 \mathrm{eV}, 1 \mathrm{GM}$, and 0.015 , which is consistent with presented results.

Including water molecules in PE more distant from protein than $3.0 \AA$ has a similar impact on $\triangle E, \sigma^{T P A}$ and $f$ values as extending the QM region (Tables S12 and S15) for the $S_{0} \rightarrow S_{1}$ transitions but the former has larger impact on spectral properties for the $S_{0} \rightarrow S_{n}$ transitions. The OPA and TPA spectra are virtually immune to QM region size with PE applied (see main text for the discussion). Thus, we believe that including more water molecules in PE force field would further support our conclusion that even chromophore alone in the QM subsystem leads to converged, with respect to QM region size, OPA and TPA spectra. On the other hand, including more water molecules in PE leads to visible increase in computational time. Thus, with more extensive QM regions, it may be highly favourable to use water molecules only in $3.0 \AA$ cut-off.

\section{S6.3 Role of effective electrostatic field in OPA and TPA spectra tuning}

According to results described in the main text, the most red-shifted and the brightest OPA band is ca. 30-40 \% darker when the MM subsystem is described at PE as compared to EE (Tables S11, S12, S14, S15, S17, and S18). Similarly, the $\sigma^{T P A}$ for the corresponding $S_{0} \rightarrow S_{1}$ transition is even 5-8 times smaller at least for avGFP-n (Tables S11-S12) while agreement between EE and PE results is much better for avGFP-a and Citrine with the anionic chromophore. Nevertheless, for some QM subsystems, the PE predicts even ca. two times smaller TPA intensity (Tables S14-S15 and S17-S18). These effects are not only due to going from the EE to more advanced PE approximation. The effective electrostatic field (EEF) [10] correction, which modifies the induced dipole moments values, ${ }^{\dagger}$ significantly damps both one- and two-photon transition intensities as deduced from data in Table S23. It was similarly the case for DsRed red fluorescent protein containing an acylimine-extended chromophore [10]. Since the absorption spectra obtained with PE do not converge to EE ones as the QM region size increases, the impact of induced dipoles modification on the absorption spectra is significantly long-range [9]. In fact, List et al. [10] report that for chromophore only in QM region, the $\sigma^{T P A}$ and $f$ are reduced from $106 \mathrm{GM}$ to $30 \mathrm{GM}$ and from 1.32 to 0.70 , respectively, upon applying the EEF correction. When the QM region is extended to 242 atoms, the EEF has a still visible but somehow smaller impact. $\sigma^{T P A}$ is reduced from $77 \mathrm{GM}$ to $42 \mathrm{GM}$, and $f$ from 1.11 to 0.80 . It suggests that EEF correction impact on OPA and TPA intensity converges very slowly with QM region size. Thus, we were unable to catch this effect with our QM subsystems combined with PE.

When the EEF correction is not applied, the OPA intensity for the $S_{0} \rightarrow S_{1}$ transition is in much better agreement with the EE results (Table S23), although it is visibly larger. In contrast, the $\sigma^{T P A}$ value is either almost two times underestimated (avGFP-n) or almost three times overestimated (avGFP-a). This suggests different response of neutral and anionic chromophore to including the mutual polarization between QM and MM regions. In case of higher excited states, the PE ansatz without EEF overshoots TPA bands intensities a few times for both chromophores.

Due to clearly long-range impact of EEF correction on absorption spectra, it should be included in PE calculations. It may be interesting to compare OPA and TPA spectra obtained with PE and PE+EEF ansatz for an ensemble of structures from MD simulation. It will serve to obtain further insight into absorption spectra tuning in this intriguing class of photoactive proteins. Such calculations will be carried in our group in the near future.

\footnotetext{
${ }^{\dagger}$ See Methodology section in the main text for more details.
} 
Table S23: Comparison of excitation energy $(\Delta E[\mathrm{eV}])$, two-photon absorption cross-section $\left(\sigma^{T P A}[\mathrm{GM}]\right)$ and onephoton oscillator strength $(f)$ obtained with $(\mathrm{PE}+\mathrm{EEF})$ and without $(\mathrm{PE})$ effective electrostatic field (EEF) correction within polarizable embedding (PE) ansatz. The electrostatic embedding (EE) results are also shown for comparison. Calculations were performed for the 0.00 QM region of avGFP-n and avGFP-a.

\begin{tabular}{|c|c|c|c|c|c|c|c|c|c|}
\hline & \multicolumn{3}{|c|}{ EE } & \multicolumn{3}{|c|}{ avGFP-n } & \multicolumn{3}{|c|}{ PE } \\
\hline State & $\Delta E$ & $\sigma^{T P A}$ & $f$ & $\Delta E$ & $\sigma^{T P A}$ & $f$ & $\Delta E$ & $\sigma^{T P A}$ & $f$ \\
\hline$S_{1}$ & 3.28 & 4.8 & 0.858 & 3.26 & 0.7 & 0.493 & 3.26 & 2.5 & 0.933 \\
\hline$S_{2}$ & 4.31 & 1.6 & 0.001 & 4.25 & 6.9 & 0.021 & 4.25 & 24.9 & 0.035 \\
\hline$S_{3}$ & 4.35 & 24.8 & 0.022 & 4.60 & 53.1 & 0.038 & 4.60 & 187.6 & 0.072 \\
\hline$S_{4}$ & 4.57 & 22.0 & 0.026 & 4.64 & 1.1 & 0.031 & 4.64 & 3.2 & 0.047 \\
\hline$S_{5}$ & 4.65 & 73.1 & 0.017 & 5.34 & 39.3 & 0.033 & 5.34 & 131.6 & 0.048 \\
\hline$S_{6}$ & 4.89 & 6.0 & 0.001 & 5.53 & 98.8 & 0.030 & 5.53 & 351.8 & 0.054 \\
\hline$S_{7}$ & 5.29 & 39.4 & 0.006 & 5.56 & 10.5 & 0.008 & 5.56 & 37.6 & 0.013 \\
\hline$S_{8}$ & 5.31 & 35.3 & 0.052 & 5.61 & 4.0 & 0.006 & 5.61 & 11.3 & 0.006 \\
\hline$S_{9}$ & 5.38 & 13.4 & 0.089 & 5.68 & 32.3 & 0.068 & 5.68 & 116.8 & 0.106 \\
\hline \multirow[t]{2}{*}{$S_{10}$} & 5.47 & 22.1 & 0.013 & 5.78 & 1.7 & 0.006 & 5.78 & 5.2 & 0.006 \\
\hline & \multicolumn{9}{|c|}{ "avGFP-a } \\
\hline State & $\Delta E$ & $\sigma^{T P A}$ & $f$ & $\Delta E$ & $\sigma^{T P A}$ & $f$ & $\Delta E$ & $\sigma^{T P A}$ & $f$ \\
\hline$S_{1}$ & 3.10 & 3.1 & 1.064 & 3.10 & 3.1 & 0.691 & 3.10 & 9.2 & 1.196 \\
\hline$S_{2}$ & 4.15 & 1.2 & 0.002 & 4.47 & 30.0 & 0.013 & 4.47 & 92.2 & 0.018 \\
\hline$S_{3}$ & 4.35 & 1.9 & 0.018 & 4.55 & 21.3 & 0.009 & 4.55 & 63.0 & 0.014 \\
\hline$S_{4}$ & 4.55 & 0.3 & 0.003 & 4.66 & 14.2 & 0.003 & 4.66 & 42.3 & 0.002 \\
\hline$S_{5}$ & 4.62 & 48.4 & 0.021 & 4.94 & 0.5 & 0.007 & 4.94 & 1.0 & 0.009 \\
\hline$S_{6}$ & 4.87 & 1.4 & 0.008 & 5.20 & 4.9 & 0.103 & 5.20 & 11.1 & 0.142 \\
\hline$S_{7}$ & 4.92 & 0.5 & 0.001 & & & & & & \\
\hline$S_{8}$ & 4.95 & 4.3 & 0.104 & & & & & & \\
\hline$S_{9}$ & 5.00 & 2.8 & 0.001 & & & & & & \\
\hline$S_{10}$ & 5.06 & 5.4 & 0.015 & & & & & & \\
\hline$S_{11}$ & 5.28 & 15.2 & 0.043 & & & & & & \\
\hline$S_{12}$ & 5.33 & 22.8 & 0.005 & & & & & & \\
\hline$S_{13}$ & 5.39 & 32.1 & 0.013 & & & & & & \\
\hline$S_{14}$ & 5.51 & 20.0 & 0.000 & & & & & & \\
\hline$S_{15}$ & 5.54 & 88.3 & 0.003 & & & & & & \\
\hline
\end{tabular}

\section{References}

[1] G. Lippert, J. Hutter, and M. Parrinello, "The Gaussian and augmented-plane-wave density functional method for ab initio molecular dynamics simulations," Theor. Chem. Acc., vol. 103, pp. 124-140, 1999.

[2] T. Laino, F. Mohamed, A. Laio, and M. Parrinello, “An Efficient Real Space Multigrid QM/MM Electrostatic Coupling," J. Chem. Theory Comput., vol. 1, pp. 1176-1184, 2005.

[3] B. Barstow, N. Ando, C. U. Kim, and S. M. Grunner, “Alteration of citrine structure by hydrostatic pressure explains the accompanying spectral shift,” Proc. Natl. Acad. Sci. USA, vol. 105, pp. 13362-13366, 2008.

[4] J. Chang, M. G. Romei, and S. G. Boxer, "Structural Evidence of Photoisomerization Pathways in Fluorescent Proteins," J. Am. Chem. Soc., vol. 141, pp. 15504-15508, 2019.

[5] T. Yanai, D. P. Tew, and N. C. Handy., "A new hybrid exchange-correlation functional using the Coulomb-attenuating method (CAM-B3LYP)," Chem. Phys. Lett., vol. 393, pp. 51-57, 2004.

[6] J. M. H. Olsen, K. Aidas, and J. Kongsted, "Excited States in Solution through Polarizable Embedding," J. Chem. Theory Comput., vol. 6, pp. 3721-3734, 2010.

[7] A. D. Laurent and D. Jacquemin, “TD-DFT Benchmarks: A Review,” Int. J. Quantum Chem., vol. 113, pp. 20192039, 2013. 
[8] D. Jacquemin, V. Wathelet, E. A. Perpete, and C. Adamo, "Extensive TD-DFT Benchmark: Singlet-Excited States of Organic Molecules," J. Chem. Theory Comput., vol. 5, pp. 2420-2435, 2009.

[9] M. T. P. Beerepoot, A. H. Steindal, K. Ruud, J. M. H. Olsen, and J. Kongsted, "Convergence of environment polarization effects in multiscale modeling of excitation energies," Comput. Theor. Chem., vol. 1040-1041, pp. 304-311, 2014.

[10] N. H. List, H. J. Aa. Jensen, and J. Kongsted, "Local Electric Fields and Molecular Properties in Heterogeneous Environments through Polarizable Embedding," Phys. Chem. Chem. Phys., vol. 18, pp. 10070-10080, 2016. 